\title{
Reinforcement Learning in Differential Games: A Learning Invader for the Guarding a Territory Game
}

\author{
by \\ Hashem Raslan, B.Sc. \\ A thesis submitted to the \\ Faculty of Graduate and Postdoctoral Affairs \\ in partial fulfillment of the requirements for the degree of
}

Master of Applied Science in Electrical and Computer Engineering

Ottawa-Carleton Institute for Electrical and Computer Engineering

Department of Systems and Computer Engineering

Carleton University

Ottawa, Ontario

May, 2015

(C) Copyright

Hashem Raslan, 2015 
The undersigned hereby recommends to the

Faculty of Graduate and Postdoctoral Affairs acceptance of the thesis

\title{
Reinforcement Learning in Differential Games: A Learning Invader for the Guarding a Territory Game
}

\author{
submitted by Hashem Raslan, B.Sc.
}

in partial fulfillment of the requirements for the degree of

Master of Applied Science in Electrical and Computer Engineering

Professor Howard Schwartz, Thesis Supervisor

Professor Sidney Givigi, Thesis Co-supervisor

Professor Roshdy Hafez, Chair,

Department of Systems and Computer Engineering 
Ottawa-Carleton Institute for Electrical and Computer Engineering

Department of Systems and Computer Engineering

\section{Carleton University}

May, 2015 


\section{Abstract}

This thesis explores the use of a learning algorithm in the "guarding a territory" game. The game occurs in continuous time, where a single learning invader tries to get as close as possible to a territory before being captured by a guard. Previous research has approached the problem by letting only the guard learn. We will examine the other possibility of the game, in which only the invader is learning. Furthermore, in our case the guard is superior (faster) to the invader. We will also consider using models with non-holonomic constraints. A control system is designed and optimized for the invader to play the game and reach the Nash Equilibrium. The thesis shows how the learning system is able to adapt to an unknown environment. We evaluated our system's performance through different simulations and compared it to the Nash Equilibrium. We conducted experiments at the Royal Military College of Canada using real robots. Our experiments verified our simulation results in a real-life environment. Our results show that our learning invader behaved rationally in different circumstances. 


\section{Acknowledgments}

I would like to thank my thesis supervisor Prof. Howard Schwartz, as well as, my co-supervisor Dr. Sidney Givigi for their support and guidance. I would also like to thank the members at the Autonomous Robotics Research Group (ARRG) at the Royal Military College of Canada for their assistance in my experiments. I would also like to acknowledge the staff and faculty of the Systems and Computer Engineering department at Carleton University that I interacted with during my studies at Carleton University. I would also like to thank the faculty of Engineering at the American University in Dubai for preparing me with the knowledge to start my graduate studies. Finally, I would like to thank my family and friends for their infinite support and encouragement during my studies in the past years. 


\section{Table of Contents}

Abstract iv

\begin{tabular}{|l|l}
\hline Acknowledgments & v
\end{tabular}

Table of Contents vi vi

List of Figures $\quad$ ix

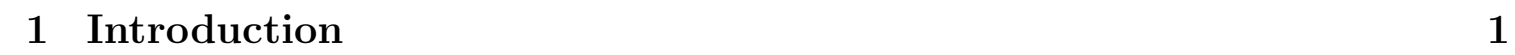

1.1 Overview . . . . . . . . . . . . . . . . . . . . . 1

1.2 Motivation . . . . . . . . . . . . . . . . . 2

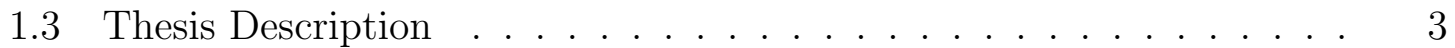

1.4 Thesis Organization . . . . . . . . . . . . . . . . . . . . . . . . 3

1.5 Contribution . . . . . . . . . . . . . . . . . 4

\begin{tabular}{|ll|}
\hline 2 & Background and Literature Review
\end{tabular}

2.1 Reinforcement Learning . . . . . . . . . . . . . . . . . . . 8

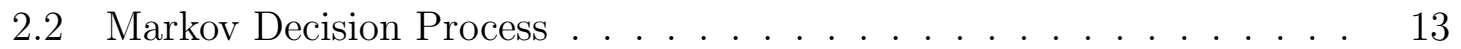

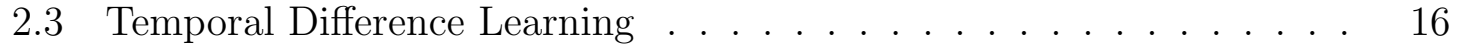

2.4 Matrix Games . . . . . . . . . . . . . . . . . . . . . . . . . . . . . . 18

2.5 Stochastic Games . . . . . . . . . . . . . . . . . . . . . 23 
2.6 Reinforcement Learning in Games . . . . . . . . . . . . . . . . . . . . 29

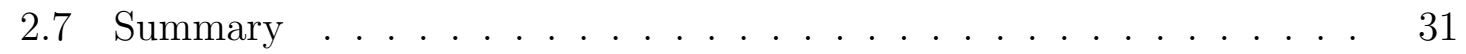

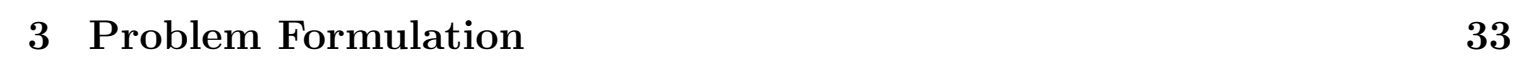

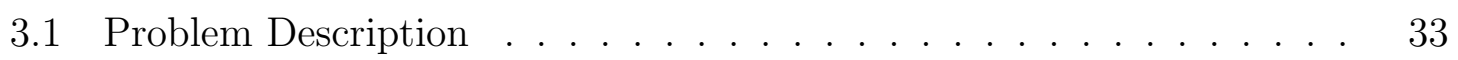

3.1 .1 Grid Game . . . . . . . . . . . . . . . . . . . . . . 34

3.2 Guarding a Territory in Differential Games . . . . . . . . . . . . . . . 38

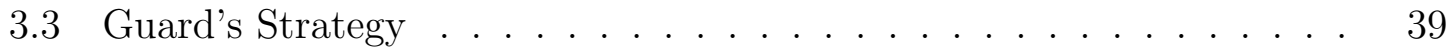

3.3 .1 Guard's Optimal Strategy . . . . . . . . . . . . . . . . . . . . 39

3.3 .2 Irrational Guard Strategy . . . . . . . . . . . . . . . . . 42

3.4 Estimating the Game State . . . . . . . . . . . . . . . . 43

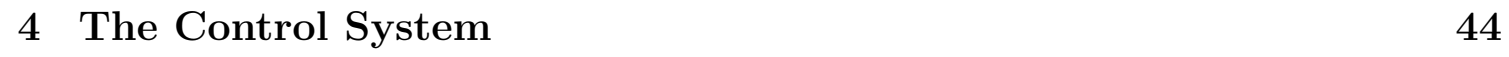

4.1 Fuzzy Logic Introduction . . . . . . . . . . . . . . . . . . . . . . . . 44

$4.1 .1 \quad$ Fuzzy Rules . . . . . . . . . . . . . . . . . . . . . 45

4.1 .2 Fuzzy System . . . . . . . . . . . . . . . . . . 47

4.2 The Fuzzy Controller . . . . . . . . . . . . . . . . . . . . . . . . . . 48

4.3 Fuzzy Q-Learning (FQL) . . . . . . . . . . . . . . . . 51

$4.4 \quad$ Fuzzy Actor Critic Learning (FACL) . . . . . . . . . . . . . . . 54

4.5 Reward Function Selection . . . . . . . . . . . . . . . . . . . 56

4.6 Summary $\ldots \ldots \ldots \ldots \ldots \ldots$

5 Simulation and Experimental Results 59

5.1 Grid World Simulation . . . . . . . . . . . . . . . . . . . . 59

5.2 Single-Player Game (Invader Reaching Territory) . . . . . . . . . . . 65

5.2 .1 Replica Controller . . . . . . . . . . . . . . . 65

$5.2 .2 \quad$ Single Input Controller . . . . . . . . . . . . . . . . . . . . 72 
$5.3 \quad$ Two-Player Game (Guarding a Territory) $\ldots \ldots \ldots \ldots \ldots$

$5.3 .1 \quad$ Optimal Guard Simulation Results $\ldots \ldots \ldots$. . . . . . 81

5.3 .2 Irrational Guard Simulation Results . . . . . . . . . . . . 85

5.4 Review of Simulation Results . . . . . . . . . . . . . . . . . . 88

$5.5 \quad$ Guarding a Territory game Experiment Setup $\ldots \ldots \ldots \ldots$

$5.6 \quad$ Experimental Evaluation of Simulation Results . . . . . . . . . . . 90

$5.7 \quad$ Online Learning $\ldots \ldots \ldots \ldots$

5.8 Discussion and Summary $\ldots \ldots \ldots \ldots \ldots$

\begin{tabular}{|ll|}
6 & Conclusion and Recommendation \\
\hline
\end{tabular}

6.1 Conclusion . . . . . . . . . . . . . . . . . . . . . 105

6.2 Contribution . . . . . . . . . . . . . . . . 106

6.3 Recommendation for Future Work . . . . . . . . . . . . . . . . 107

\begin{tabular}{ll}
\hline List of References & 110
\end{tabular} 


\section{List of Figures}

2.1 Reinforcement Learning _ . . . . . . . . . . . . . . . . . . 8

$2.2 \quad$ Route Selection Strategy $\ldots \ldots \ldots \ldots$

$2.3 \quad$ Average reward $n$-armed bandit problem $\ldots \ldots \ldots \ldots$

2.4 Markov Decision Process . . . . . . . . . . . . . . . . . . . . . 14

$3.12 \times 2$ Grid Game $\ldots \ldots \ldots \ldots \ldots$

3.2 Grid Game - Non-Terminal States . . . . . . . . . . . . . . . . 36

3.3 Grid Game - Terminal States . . . . . . . . . . . . . . . . . 36

3.4 State Transition Probabilities $\ldots \ldots \ldots \ldots \ldots$

3.5 Game Environment . . . . . . . . . . . . . . . . . . . . . . . . . . . 39

$3.6 \quad$ Reachable region geometry $\ldots \ldots \ldots \ldots$

$3.7 \quad$ Guard's Optimal Strategy - Nash Equilibrium . . . . . . . . . . . 42

$4.1 \quad$ Fuzzy System $\ldots \ldots \ldots \ldots \ldots$

4.2 angle $\theta$ between invader and territory $\ldots \ldots \ldots \ldots \ldots$

$4.3 \quad$ Manhattan distance between guard and invader $\ldots \ldots \ldots \ldots$

$4.4 \quad$ Membership function for the angle input . . . . . . . . . . . 51

$4.5 \quad$ Membership function for Manhattan distance input . . . . . . . . . 52

$4.6 \quad$ Actor Critic Logic System Diagram . . . . . . . . . . . . . . 55

5.1 Grid World Q-Minimax Simulation Result - Guard's Strategy _. . . 60

5.2 Grid World Q-Minimax Simulation Result - Invader's Strategy . . . . 61 
5.3 Grid World WoLF-PHC Simulation Result - Guard's Strategy . . . . 62

5.4 Grid World WoLF-PHC Simulation Result - Invader's Strategy. . . . 63

5.5 Grid World Q-Minimax Simulation Result - Invader's Strategy vs. Guard's pure strategy . . . . . . . . . . . . . . . . . . . . . 64

5.6 Grid World WoLF-PHC Simulation Result - Invader's Strategy vs. Guard's pure strategy . . . . . . . . . . . . . . . . . . . . . . 64

$5.7 \quad$ Membership function using coordinates signals as inputs $\ldots \ldots 6$

5.8 FQL - Invader Reaching Territory - Epoch 001 . . . . . . . . . . . 68

$5.9 \quad$ FQL - Invader Reaching Territory - Epoch 400 . . . . . . . . . . . 68

5.10 FQL - Invader Reaching Territory - Payoff . . . . . . . . . . . . . 69

5.11 FACL - Invader Reaching Territory - Epoch 001 . . . . . . . . 70

5.12 FACL - Invader Reaching Territory - Epoch $400 \ldots \ldots \ldots$

5.13 FACL - Invader Reaching Territory - Payoff . . . . . . . . . . . 71

5.14 Termination Time average - FQL vs. FACL $\ldots \ldots \ldots \ldots . \ldots 72$

5.15 FQL - Invader Reaching Territory - Single Input Controller - Epoch 00173

5.16 FQL - Invader Reaching Territory - Single Input Controller - Epoch 40074

5.17 FQL - Invader Reaching Territory - Single Input Controller - payoff . 75

5.18 FACL - Invader Reaching Territory - Single Input Controller - Epoch

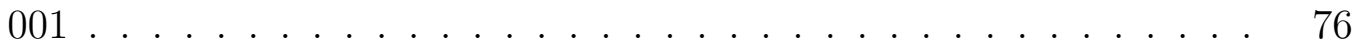

5.19 FACL - Invader Reaching Territory - Single Input Controller - Epoch

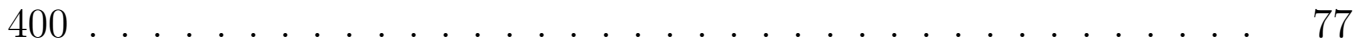

$\begin{array}{lll}5.20 & \text { FACL - Invader Reaching Territory - Single Input Controller - payoff } & 77\end{array}$

5.21 Termination Time average - FQL vs. FACL - Single Input Controller 78

5.22 Training region $\ldots \ldots \ldots \ldots \ldots \ldots \ldots \ldots$

5.23 Invader vs. Optimal Guard - before learning . . . . . . . . . . . 82

5.24 Learning Invader vs. Optimal Guard - first test epoch results . . . . . 82 
5.25 Learning Invader vs. Optimal Guard - final test epoch results . . . . 83

5.26 Learning Invader vs. Optimal Guard - Invader's payoff . . . . . . . . 83

5.27 Learning Invader vs. Optimal Guard - possible reaching test . . . . . 85

5.28 Learning Invader vs. Irrational Guard - first test epoch results . . . . 86

5.29 Learning Invader vs. Irrational Guard - final test epoch results . . . . 87

5.30 Learning Invader vs. Irrational Guard - Invader's payoff . . . . . . . . 88

5.31 HUSKY Robot specs . . . . . . . . . . . . . . . . . . . . . . . . . . . 89

5.32 Experiment Setup . . . . . . . . . . . . . . . . . . . . . . . . . . . 90

5.33 Experiment Trajectories: Learning Invader vs. Optimal Guard . . . . 91

5.34 Experiment Trajectory: Learning Invader vs. Irrational Guard . . . . 92

5.35 Experiment Trajectories: Both players are following Nash Equilibrium 93

5.36 Experiment Trajectories: Irrational Guard vs. Invader doing Nashstrategy for an optimal guard . . . . . . . . . . . . . . . . . . . . . . 94

5.37 Experiment Environment: trackable region . . . . . . . . . . . . . . . 95

5.38 Online Learning: Learning Invader vs. Optimal Guard - first experiment 97

5.39 Online Learning: Learning Invader vs. Optimal Guard - Last experiment 98

5.40 Online Learning: Learning Invader vs. Optimal Guard - payoff . . . . 98

5.41 Online Learning: Learning Invader vs. Irrational Guard - first experiment 99

5.42 Online Learning: Learning Invader vs. Irrational Guard - Last experiment ........................ . . 100

5.43 Online Learning: Learning Invader vs. Irrational Guard - payoff . . . 100

5.44 Alternative Training Region . . . . . . . . . . . . . . . . . 103 


\section{Chapter 1}

\section{Introduction}

\subsection{Overview}

Computer devices were initially designed to perform a specific single task, such as calculating the product of two large numbers. In 1939, Bell Laboratories developed the Complex Number Calculator. Computer devices have advanced to become readjustable and reprogrammable. They can now perform more complex tasks. With the advances in technology, the use of computer systems expanded in many different fields. One of those fields is Robotics. Robots are mechanical machines that are controlled by a computer system. The computer system controls the robots by sending commands for the machine to actuate its motors. We use robot to ease humans' lives and accomplish tasks we are unable to do.

The human mind is incredibly fascinating. We are still unable to know everything about how our brains work. The brain enables the human-being to perform physical actions, think of ideas, make decisions, and feel emotions. Most importantly, the mind enables us to learn. We take our actions based on what we have learnt. However, some of our everyday decisions are based on our instincts and feelings.

With the breakthroughs in embedded microcontrollers and digital technology, 
computer devices that could have taken an entire room several decades ago can now be placed on smaller and faster devices that consume less power. The technological advances led scientists and engineers to focus on creating a computer system that can replicate the human mind. This created the field of Machine Intelligence.

Machine Intelligence (Artificial Intelligence - AI) was first introduced in the mid 1900s. It aims at enabling the computer device to learn from its experience. Machine intelligence creates an adaptive learning system. Machine intelligence does not only belong to the field of robotics, but it is spread in many other uses, such as modern video games.

Different learning algorithms have been previously developed. In this thesis we focus on Reinforcement Learning algorithms. The scope of this thesis includes investigation on Reinforcement Learning algorithms including Q-Minimax, WoLF-PHC, FQL and FACL algorithms. In this thesis we look into the use of reinforcement learning algorithm in the "Guarding a Territory" game.

\subsection{Motivation}

The increase in demand for home security 1 , as well as, the development of robotic systems make the idea of having an autonomous guarding robot not far from reality. "Guarding a territory" is a game with two sets of players; Guards and Invaders. The guards try to protect a certain space, the territory, which the invaders are trying to reach. This game was first described by Isaacs [1]. Different applications for this game can be found in crucial real-life scenarios, such as protecting borders from smuggling or breaching a secure space. Some papers have been published that investigate the "guarding the territory" game [2] [3]; however, they mainly focus on the use of fixed

\footnotetext{
1“The Demand for Security" IBM Global Business Service, Bethesda MD. May 2008.
} 
algorithms.

This thesis will investigate the use of Reinforcement Learning algorithm that can easily adapt itself and perform optimally to different strategies taken by the opponent. An invading system that can easily adapt itself to the current environment has an important use. It can be used as a testing mechanism to evaluate how successful and strong is a guarding system. Once the invader learns the guard's weakness, the guard's weakness can be exploited.

\subsection{Thesis Description}

We are going to look at a differential version of the "guarding a territory game" with a single guard and a single invader. The game will occur in a bounded space with no obstacles. The guard is going to follow different fixed strategies. The invader will learn as it plays the game. We will build a customized control system and combine it with Reinforcement Learning to build our learning invader. We expect the invader to learn and perform rationally versus the different strategies performed by the guard.

\subsection{Thesis Organization}

- Chapter 2 will provide a brief background on the important topics used to complete the thesis. It will introduce Reinforcement Learning. Then it will provide the important notations and definition that will be used throughout the thesis. Finally, it will represent the Reinforcement Learning problem in terms of Game Theory analogy.

- Chapter 3 will provide a formal representation of the problem being addressed. It will also highlight the work done in literature. 
- Chapter 4 will describe our proposed solution and control system in details.

- Chapter 5 will contain the simulation and experimental results with some analysis and discussion over the results.

- Chapter 6 will contain a summary for the work accomplished, followed by a conclusion and suggestion for future work.

\subsection{Contribution}

In this thesis we try to increase the complexity of the game in comparison to what already exists in the field. Existing work has investigated the problem in different environments. In [2], the game of guarding a line segment with a simple action space for both the guard and the invader to move along a straight line is described. Work done by [4] has investigated the "guarding a territory game", but in a $6 \times 6$ grid game. Differently, we look into a larger environment space in continuous time with continuous action.

Work done by [5] considered a differential version of the game. However, it considered holonomic kinematics for both players and both players had equal kinematics. Similar model characteristics were used by [3] and [6]. We look into a more realistic model, which is done by using a cart model with non-holonomic constraints. The research described in [5] and [4] used a learning algorithm to create their agent strategy. In [5], the author only let the guard learn, but the invader was following a preset algorithm. We look at the other side of the game, such that the invader is going to learn.

The contributions of this thesis can be described as:

1. Building a customized fuzzy control system to guide the invader. The control 
system is optimized for the invader to be used with Reinforcement Learning and to direct the invader.

2. Proposing an instantaneous reward function that allows the invader to learn in the game.

3. Simulation and comparison between the FQL and FACL algorithm for a simplified version of the game with only 1 invader using non-holonomic constraints.

4. Simulation of the "Guarding a Territory" game and demonstration of the learning agent performance with a faster (superior) guard and with non-holonomic constraints.

5. Evaluation of the simulation results through experiments in laboratory environment using real robots.

6. "Guarding a Territory" game experiment on real robots and demonstration of the learning agent ability to learn in a real-life scenario. 


\section{Chapter 2}

\section{Background and Literature Review}

Machine learning is the ability for a program to develop, based on the current observation of environment, and behave intelligently (rationally) [7]. There are two types of learning in machine intelligence; supervised and unsupervised. Supervised learning depends on previous knowledge of the environment and action space. Despite that supervised learning is beneficial, it would be unable to learn from interaction with unknown environment when the correct action is unknown. In this condition, Reinforcement Learning (RL), a type of unsupervised learning, is useful [8].

RL has started in the early 1980s. The RL was developed from trial-and-error learning, Markov Decision Process (MDP) and Temporal Difference (TD) [9]. The MDP deals with representing the environment in discrete states as will be explained in Sec. 2.2. The TD deals with evaluating the discrete states in the MDP. The MDP combined with TD can be used to produce a learning agent. The trial-and-error learning means that the learning agent will learn from its mistakes. It emerged from the psychology of animal learning [8]. The three elements produce the RL techniques. RL focuses on providing the learning agent with an optimal control. The optimal control enables the agent to perform its task efficiently.

Robots utilized with RL are learning agents. Robots can observe the environment 
using sensors. The agent's observations are the agent's situation in a problem. RL maps situations to actions. It aims at creating a goal-seeking agent [8]. RL is based on rewards and punishments, which are given based on how the learning agent acts to reach its goal $[9]$.

Due to the MDP, RL is limited with discrete state perception and discrete actions. Thus, it becomes complex for an agent to learn when many variables need to be adapted. This made researchers combine Fuzzy Inference Engines with RL [10]. In recent years, RL algorithms have been widely used in coordinating multiple robots to explore a space [11]. RL has been used as an AI tool to produce autonomous agents that can organize their own system. This is done to enable robots to interact with environments with no prior knowledge [12]. In literature, researchers have combined RL with fuzzy controllers to deal with large space and noisy environments [13] [14.

Game theory is a branch of mathematics that aims at making decisions when agents are in conflicts [15]. RL has been recently applied to game theory to enable the learning agents to make the correct decisions. From that, researchers started using RL in differential and cooperative gaming. In Sec. 2.6, we describe some of the usages of RL in cooperative gaming. In this thesis, we look into a single agent that learns using Reinforcement Learning to play in a 2-player game.

The purpose of this chapter is to introduce and discuss the major elements and characteristics that were used to build our learning agent. The first section gives an introduction on Reinforcement Learning. The second and third section introduces the Markov Decision Process and describes how to model the problem in terms of states. The fourth and fifth sections show how the reinforcement learning problem can be described in terms of game theory. The last two sections will include some of the usages of Reinforcement Learning in literature and a summary. 


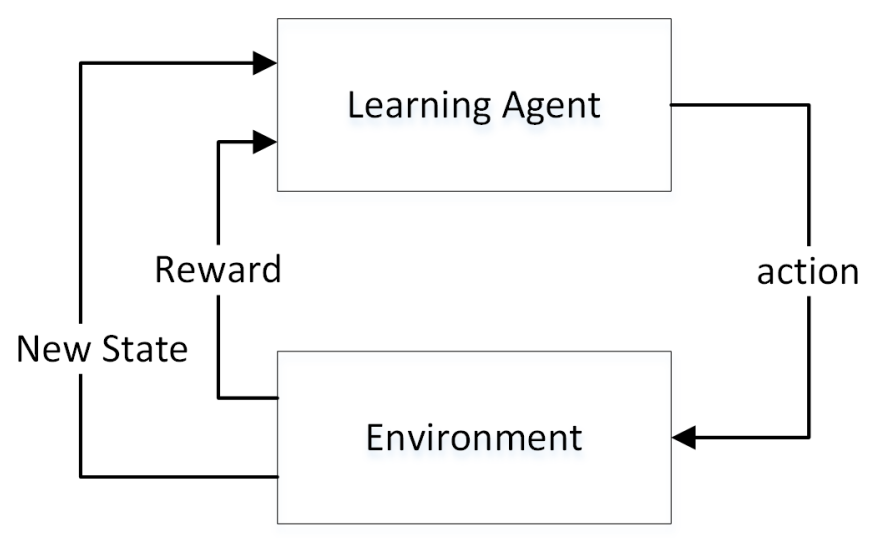

Figure 2.1: Reinforcement Learning

\section{$2.1 \quad$ Reinforcement Learning}

The RL helps the learning agent to perform its goals throughout the agent's interactions with the environment. RL focuses on increasing the agent's reward. The reward describes how well the agent is performing to reach its goals. The RL algorithm has to create a balance between exploration and exploitation; meaning the learning agent has to remember how successful were the actions previously taken and repeat them (exploitation). Also, the agent should occasionally take random actions (explorations). This is done to ensure trying different action scenarios and not getting stuck in a local-minima/maxima. The overall output of RL is empowering the learning agent with a sufficient strategy to perform the agent's purpose [8].

On the assumption that we have a single learning agent, we describe the environment as everything that surrounds the agent and everything that interacts with it. The agent's current state $s_{t}$ is the condition of the agent and the environment at the current time $t$. For one to perform a certain task (goal), one has to take a set of actions. Actions create events in the environment and change the state. Figure 2.1 shows the agent-environment interaction in reinforcement learning. The agent takes a certain action $a_{t}$ (or a set of actions). The agent then observes its new state $s_{t+1}$ 


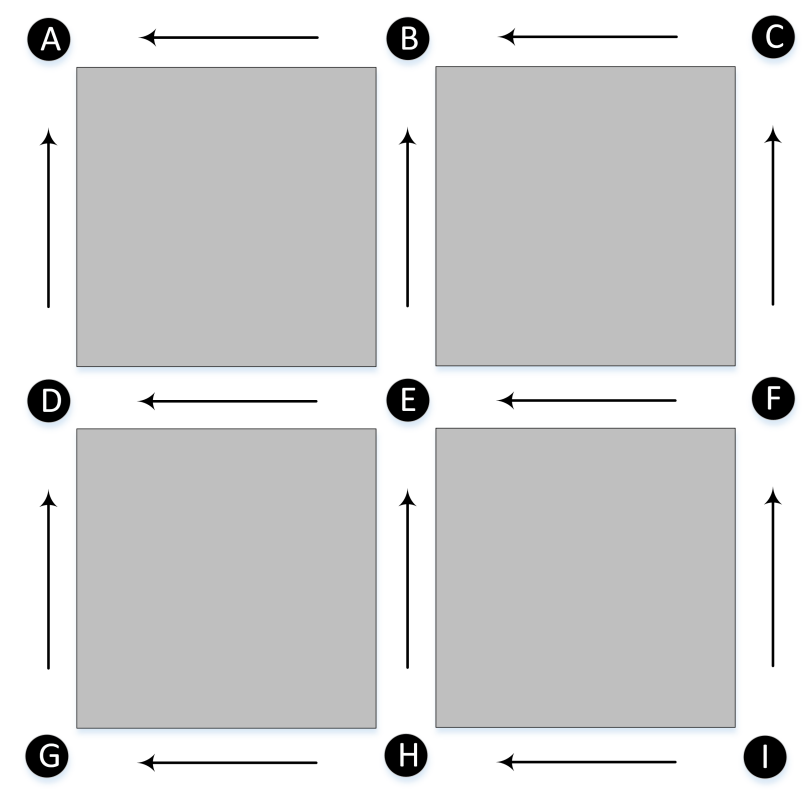

Figure 2.2: Route Selection Strategy

after taking that action. The agent also receives a reward $r_{t+1}$. This reward acts as a feedback on how successful are the actions taken. They help the agent in knowing how useful were the actions taken. Rewards can be delayed or instantaneous.

The set of actions the agent takes describes the agent's strategy. A strategy is how we plan to achieve a certain goal. Different strategies can perform the same goal, but not all strategies are optimal.

Consider an example of a person trying to navigate around the city. Assuming that person is trying to reach, the fastest, from point "I" to point "A" as seen in Figure 2.2, where the distance and speed between all intersections are equal. Intersections (points) are represented by black dots. This examples assumes that the person will only choose the actions that will get him/her closer to their destination. The available actions will become up or left at each intersection as displayed by arrows in the same figure.

In that case, reaching point "A" will always take 4 actions and 3 intermediate points. 
This creates a set of possible strategies that the person can follow. However, what if there's traffic at intersections "E" and "B" that slows the speed in the connected routes? Even though all strategies will lead to the same target, only one route ("I" $\rightarrow$ "H" $\rightarrow$ "G" $\rightarrow$ "D" $\rightarrow$ "A") will be faster (optimal). We define that the minimum possible time to reach the target as optimal. In other cases, optimality can be described differently.

Similarly, the agent takes a set of actions to perform a goal. The agent takes those actions based on his current state and what it has previously learnt. The actions the agent takes describes the agent's strategy. The current state can be the agent's position, as in the previous example. The information learnt can be how to avoid the traffic.

A policy, $\pi_{t}$, describes the strategy the agent will follow at time $t$. This is done by giving a probability of following a specific action (from the action set). This probability represents how successful an action is. The probability should also get updated based on the agent's new interactions; consequently, the agent's policy will get updated over time. In other words, an agent following policy $\pi_{t}$ will take action $a_{t}=a$ while in state $s_{t}=s$ based on the probability described by $\pi_{t}(s, a)$.

The optimal policy $\pi^{*}(s)$ describes the best policy an agent can have to achieve the same task. Optimality depends on the goal description. The RL can enable the learning agent's policy to get very close to the optimal policy, if not achieve it.

As previously described, RL focuses on increasing the agent's reward. Increasing the reward depends on the nature of the task being done. If the task completes in a finite time, then the total rewards received $R_{\text {total }}$ can be the sum of all rewards as seen in 2.1), such that $t$ is time step and $T$ is termination time:

$$
R_{\text {total }}=\sum_{t=1}^{T} r_{t}
$$


In this case, the expected maximum future rewards at time step $t$ is as defined in (2.2):

$$
R_{t}=\sum_{k=1}^{T} r_{t+k}
$$

In other cases, the task continues indefinitely. Therefore, it becomes necessary to try to maximize the discounted future rewards as shown in (2.3):

$$
R_{t}=\sum_{k=0}^{\infty} \gamma^{k} r_{t+k+1}
$$

Such that $\gamma$ is positive and less than 1 . The agent should always try to maximize $R_{t}$ at every time step. The larger $\gamma$ is, the further the agent focuses on the future. The smaller $\gamma$ is, the further it focuses on the current reward and becomes a myopic system [8].

\section{$n$-Armed Bandit Problem}

To be able to elaborate more on how RL can be used, we look at the " $n$-Armed Bandit" problem. This is a case with having n-arms on the slot machine. Pulling an arm results in a reward, which may be negative. Each arm is considered a different action. In this case, the total reward will become the average of rewards received. The true value of an action $\left(Q^{*}(a)\right)$ can be estimated at time $t$ by the average rewards received by that action as shown in (2.4), where $k_{a}$ is the number of times action $a$ was chosen [16].

$$
Q(a)=\frac{r_{1}+r_{2}+r_{3}+\ldots+r_{k a}}{k_{a}}
$$

Let's assume an example with $n=10$, such that:

$$
Q^{*}=[-0.68,-0.57,-0.75,2.18,-0.71,0.43,-0.64,-0.61,0.37,-1.50]
$$


and the agent's current estimate is,

$$
Q_{t}=[0.33,0.65,0.76,-1.12,0.01,-0.05,-0.07,1.33,0.84,-0.93]
$$

The reward received, from action $a$, is a random value from a Gaussian distribution with variance 1 , and mean $Q^{*}(a)\left(\mathcal{N}\left(Q^{*}(a), 1\right)\right)$. The agent does not know the true action value, but only its estimate. If the agent only takes the highest value estimated $(a=8)$, it is called the agent is taking the "greedy" choice. However, if the agent occasionally takes actions at random, it will guarantee a higher reward return. We define a new variable, $\epsilon$, which is the exploration rate. It is the probability of taking a random action.

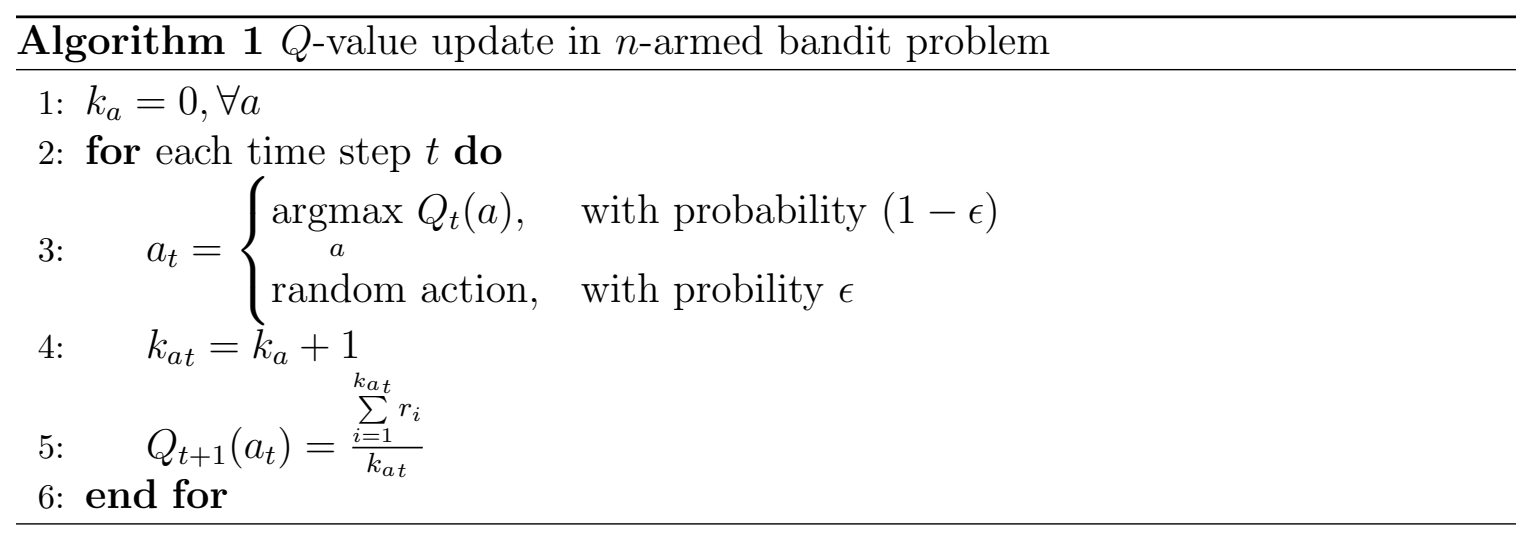

We simulate the problem 3 times following algorithm 1 . Each simulation will use different exploration rates from the following set $[0,0.01,0.1]$. Each simulation consists of 1000 plays. Figure 2.3 shows the results of the simulations. Such that, the $\mathrm{x}$-axis represents the play number and the $\mathrm{y}$-axis represents the average received reward. It can be observed that the greedy action resulted in the lowest reward return, in comparison to other exploration rates. Nonetheless, the highest possible reward can be reached with some exploration. The agent should always have a trade-off 


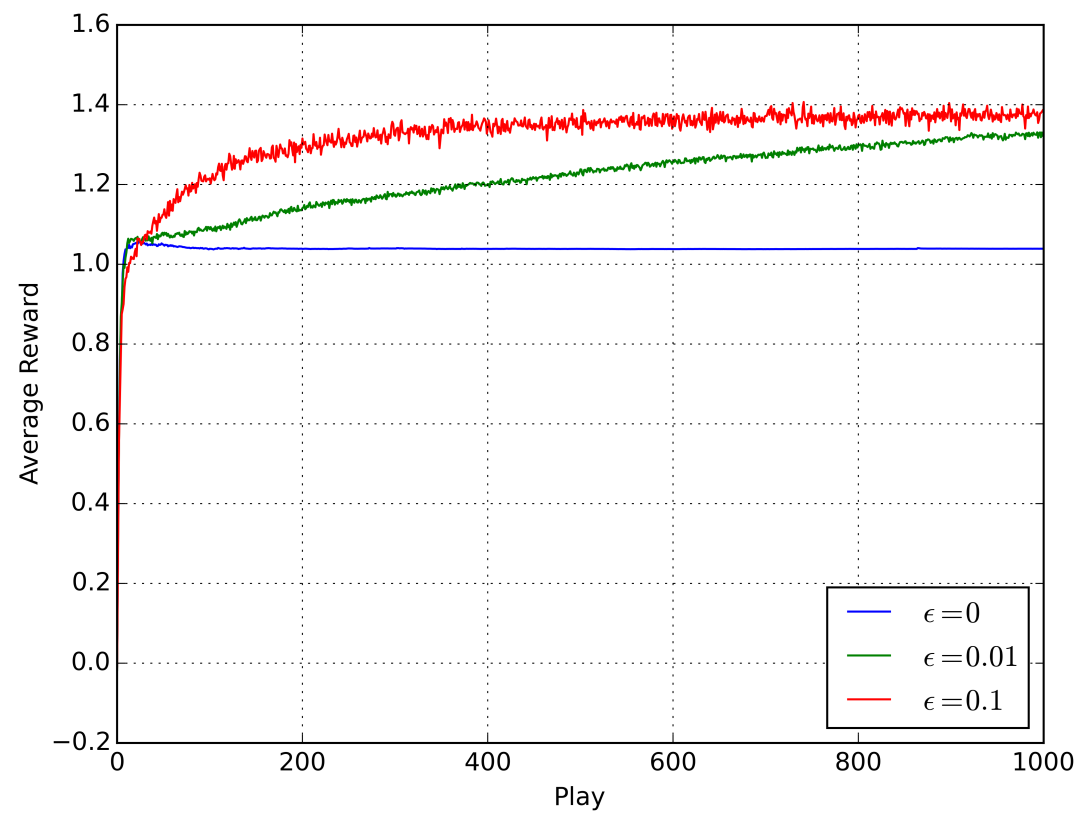

Figure 2.3: Average reward $n$-armed bandit problem

between exploration and exploitation.

\subsection{Markov Decision Process}

In a Markov Decision Process (MDP), the current state will determine the future rewards of the task. It does not depend on the past [8]. Any MDP is defined by the five-tuple $(S, A, P, R, D)$ for a single agent. Such that $S$ is the state space $\left(s, s^{\prime} \in S\right)$, $A$ is the action space $(a \in A)$, and $R_{s, s^{\prime}}^{a}$ is the reward received from transition from state $s$ to state $s^{\prime}$ using action $a$ [17] [18. Where $D \subset A \times S$ is the set of admissible state-action pares at time $t$. Figure 2.4 illustrates the MDP. Any MDP requires the agent-environment to have the following characteristics 19]:

- The agent has to move in a sequence of states

- The state of the agent is always known

- Actions results in transition between states 


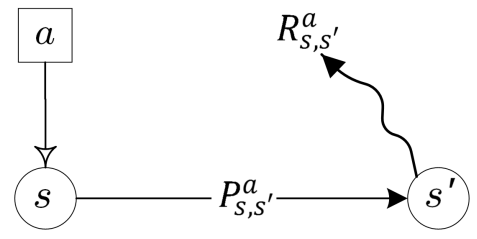

Figure 2.4: Markov Decision Process

- Actions results in rewards

The MDP makes it a lot easier to model real-life scenarios when the state and action spaces are large. It also helps the agent select the correct action at every time step, therefore, creating the agent's strategy. The probability to move from state $s$ to state $s^{\prime}$ by taking action $a$ at time $t$ can be defined as,

$$
P_{s, s^{\prime}}^{a}=\operatorname{Pr}\left\{s_{t+1}=s^{\prime} \mid s_{t}=s, a_{t}=a\right\}
$$

Next, we define the expected reward for moving from state $s$ to $s^{\prime}$ by taking action $a$ at time $t$ as shown in (2.6),

$$
R_{s, s^{\prime}}^{a}=E\left\{r_{t+1} \mid s_{t}=s, a_{t}=a, s_{t+1}=s^{\prime}\right\}
$$

Any MDP focuses only on future rewards, thus one can compute the value of a state $s$ (under policy $\pi$ ) as the expected future rewards $R_{t}$ as shown in (2.7). Where $t$ is the current time for state value evaluation and $s_{t}$ denotes the state at time $t$.

$$
\begin{array}{rc}
V^{\pi}(s) & =E_{\pi}\left\{R_{t} \mid s_{t}=s\right\}, \\
V^{\pi}(s) & =E_{\pi}\left\{\sum_{k=0}^{\infty} \gamma^{k} r_{t+k+1} \mid s_{t}=s\right\}
\end{array}
$$


The state value function creates a comparison between different states for a given policy $\pi$. Moreover, we define the action-state value of a state $s$ when taking action $a$ as $[20]$,

$$
Q^{\pi}(s, a)=E_{\pi}\left\{\sum_{k=0}^{\infty} \gamma^{k} r_{t+k+1} \mid s_{t}=s, a_{t}=a\right\}
$$

Such that $s_{t}$ and $a_{t}$ are the state and action at time $t$. Where the current state and action are $s$ and $a$.

The only difference between $Q$ and $V$ is that $Q$ considers the current actions [16]. We can rewrite the state value as shown in (2.9) [16].

$$
\begin{aligned}
& V^{\pi}(s)=\quad E_{\pi}\left\{r_{t+1}+\sum_{k=1}^{\infty} \gamma^{k} r_{t+k+1} \mid s_{t}=s\right\} \\
& =\quad E_{\pi}\left\{r_{t+1}+\gamma \sum_{k=0}^{\infty} r_{t+k+2} \mid s_{t}=s\right\} \\
& =\sum_{a} \pi(s, a) \sum_{s^{\prime}} P_{s, s^{\prime}}^{a}\left[R_{s, s^{\prime}}^{a}+\gamma E_{\pi}\left\{\sum_{k=0}^{\infty} \gamma^{k} r_{t+k+2} \mid s_{t+1}=s^{\prime}\right\}\right]
\end{aligned}
$$

Where $s^{\prime}$ is the new state and $\gamma$ is the forgetting factor.

$$
V^{\pi}(s)=\sum_{a} \underbrace{\pi(s, a)}_{\begin{array}{c}
\text { probability of tak- } \\
\text {-ing action } a
\end{array}} \sum_{s^{\prime}} \underbrace{P_{s, s^{\prime}}^{a}}_{\begin{array}{c}
\text { state transition } \\
\text { probability }
\end{array}}(\underbrace{R_{s, s^{\prime}}^{a}}_{\begin{array}{c}
\text { Reward for taking } \\
\text { action } a \\
\text { and moving from } \\
s \text { to } s^{\prime}
\end{array}}+\gamma \underbrace{V^{\pi}\left(s^{\prime}\right)}_{\begin{array}{c}
\text { New state } \\
\text { value }
\end{array}})
$$

The state value function describes how good is a state, in comparison to other states, under a specific policy. It informs the agent about the expected rewards from the state. Therefore, if the agent targets the states with higher state-value, it will be following the greedy action. 
An optimal state value function is the highest value any state can reach, under a specific policy, and gives the agent the highest reward possible(2.10). This is defined as The Optimality Principle for Infinite-Horizon MDPs [19].

$$
V^{*}(s)=\max _{\pi} V^{\pi}(s)
$$

Similarly, the optimal state-action value for taking action $a$ is:

$$
Q^{*}(s, a)=\max _{\pi} Q^{\pi}(s, a)
$$

\subsection{Temporal Difference Learning}

Dynamic programming [21] can be used to compute an optimal state value function associated with a specific policy $\pi$. However, it needs to know in advance the transition probabilities $\left(P_{s, s^{\prime}}^{a}\right)$ and the expected rewards $\left(R_{s, s^{\prime}}^{a}\right)$. When those elements are unknown, the agent can learn the best actions to take through using Temporal Difference(TD) learning or Monte Carlo Methods [8] [22]. In this thesis we use TD learning. TD can be described as bootstrapping. It uses its estimates to produce new estimates 8 .

Since moving from a state to another state results in a reward and the value of each state should be equal to the expected future reward at this state, then the current state value can be represented as in (2.12):

$$
\underbrace{V(s)}_{\begin{array}{c}
\text { Current State } \\
\text { Value }
\end{array}}=\underbrace{r_{t+1}}_{\begin{array}{c}
\text { Immediate } \\
\text { Reward }
\end{array}}+\gamma \underbrace{V\left(s^{\prime}\right)}_{\begin{array}{c}
\text { Next State } \\
\text { Value }
\end{array}}
$$

Such that $\gamma$ is the forgetting factor. 
The temporal difference $\Delta$ is then defined as shown in (2.13), such that it should be zero 8$]$.

$$
\Delta=r_{t+1}+\gamma V\left(s^{\prime}\right)-V(s)
$$

Once the temporal difference is calculated, the state value function can be updated using learning rate $\alpha$ as shown in (2.14).

$$
V_{k+1}(s)=V_{k}(s)+\alpha\left(r_{t+1}+\gamma V_{k}\left(s^{\prime}\right)-V_{k}(s)\right)
$$

The same thing can be done for the state-action value function:

$$
Q_{k+1}(s, a)=Q_{k}(s, a)+\alpha\left(r_{t+1}+\gamma Q_{k}\left(s^{\prime}, a^{\prime}\right)-Q_{k}(s, a)\right)
$$

The learning rate $\alpha$ controls how fast the agent updates its function. A learning rate of zero means the value function is not being updated. Also, a very large learning rate may cause the agent's strategy to oscillate. A constant value can be used for the learning rate; however, results by 8$]$ show that the temporal difference might not converge using a constant learning rate. This is because the agent will keep relearning what it already knows. Therefore, using a decaying learning rate is more useful [23].

\section{Q-Learning}

Q-Learning enables the agent to approximate $Q$ to the optimal policy $Q^{*}$ through regression [24] 8]. Q-Learning is described as Off-Policy TD Control. It is described as Off-Policy because it does not depend on the policy to take its actions. It has the following benefits in comparison to on-policy control:

- The learning no longer depends on the transition probabilities

- The learning no longer depends on the policy to take actions 
- The learning no longer depends on the expected rewards

- The learning follows the greedy-action when observing the Q-table (no exploration)

Given learning rate $\alpha$ and forgetting factor $\gamma$, the one-step Q-learning becomes [8],

$$
Q\left(s_{t}, a_{t}\right)_{k+1} \leftarrow Q_{k}\left(s_{t}, a_{t}\right)+\alpha\left[r_{t+1}+\gamma \max _{a} Q_{k}\left(s_{t+1}, a\right)-Q_{k}\left(s_{t}, a_{t}\right)\right]
$$

In Q-Learning, the policy gets updated based on the current $Q$. The Q-Learning algorithm is illustrated in algorithm 2 [25].

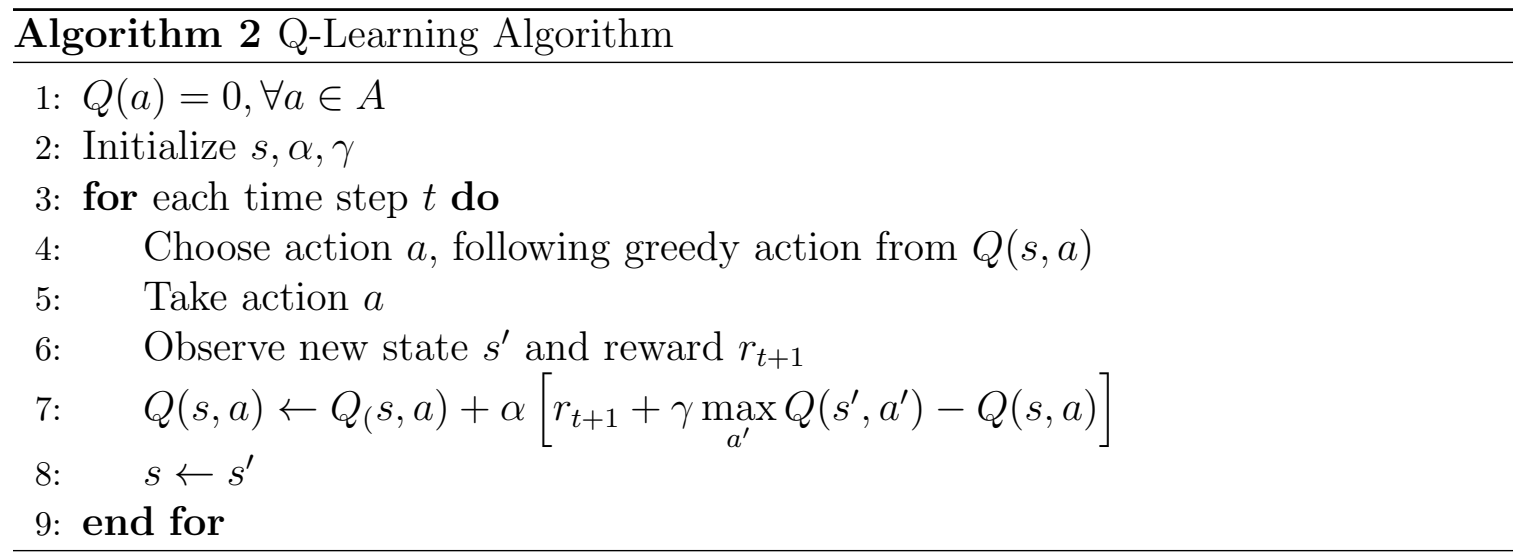

\subsection{Matrix Games}

Definition "Antagonistic games in which each player possesses a finite number of strategies are called matrix games". 26

A game is described antagonistic when it consists of two players (agents) and if following a certain strategy will always result in the same outcome. As previously discussed, MDP considers an environment with a single agent and multiple states. 
Differently, Matrix Games considers multiple players; however, with a single state. In this case, the game is our environment [16].

In a two-player matrix game, we can assume the first and second player strategies are represented by the rows and columns of a matrix. The different possible results of the game will be represented by the payoff matrix $R$ :

$$
R=\left[\begin{array}{ccccc}
r_{11} & r_{12} & r_{13} & \ldots & r_{1 n} \\
r_{21} & r_{22} & r_{23} & \ldots & r_{2 n} \\
\vdots & \vdots & \vdots & \ddots & \vdots \\
r_{m 1} & r_{m 2} & r_{m 3} & \ldots & r_{m n}
\end{array}\right]
$$

The matrix elements $r_{i j}$ (for any $i=1, \ldots, m$ and $j=1, \ldots, n$ ) in the matrix represent all the different outcomes of the game. The elements $(i, j)$ denote the strategy followed by the corresponding player.

An example of two-player matrix games is the "stone-paper-scissors" game. Each player has 3 different actions (strategies) $[1,2,3]=[$ stone, paper, scissors]. Stone beats scissors, paper beats stone, and scissors beats paper. The game payoff matrix can be represented by (2.17). Player 1 win is indicated by +1 , player 2 win is indicated by -1 , and a tie is indicated by a 0 .

$$
R=\left[\begin{array}{ccc}
0 & -1 & +1 \\
1 & 0 & -1 \\
-1 & 1 & 0
\end{array}\right]
$$

An example of another game which is more complex is tic-tac-toe [27. 
The simplest matrix game is two-player zero-sum game. In these games if player 1 receives a positive reward $(+|r|)$, player 2 receives a negative reward $(-|r|)$ and viceversa. In other words, player 1 gains are player 2 losses and vice-versa. Therefore, player 1 will try to maximize the payoff $r_{i j}$. In contrast, player 2 will try to minimize $r_{i j}[28$. For the rest of this section, we represent player 1 by a male (he, his) and player 2 by female (she, her $)$. The following is valid when $\left(R_{1}=R\right)$ and $\left(R_{2}=-R\right.$ or $R_{2}=-R^{T}$ ) represent the payoff matrices of player 1 and player 2 consecutively.

Assuming player 1 chooses strategy $(i)$, this makes his minimum possible payoff (the worst he can do) for any strategy followed by player 2 as:

$$
\text { player1 payoff } \min _{1 \leq j \leq n} \min _{1 j}
$$

Therefore, player 1 should select his strategy $i$ to maximize (2.18). Hence, his payoff (his minimax) will become:

$$
\text { player1 payoff } f_{\max -\min }=\max _{1 \leq i \leq n} \min _{1 \leq j \leq n} r_{i j}
$$

Alternatively, player 2 is trying to minimize $r_{i j}$. Hence, her maximum possible payoff (the worst she can do) becomes:

$$
\text { player2 payoff }_{\max }=\max _{1 \leq i \leq m} r_{i j}
$$

Therefore, she has to select strategy $j$ to minimize 2.20$)$. This will let her achieve a payoff (her minimax) at least lower than:

$$
\text { player2 payoff } f_{\min -\max }=\min _{1 \leq j \leq n} \max _{1 \leq i \leq m} r_{i j}
$$


Definition A situation, which is admissible for all the players is called and equilibrium situation 26.

A saddle point of the game happens when the minimax of both players is equal as seen in 2.22 . This will describe the equilibrium situation of the game. We can mark the strategies that result in the equilibrium by $i^{*}$ and $j^{*}$. The equilibrium situation can also be denoted by 2.23 .

$$
\begin{gathered}
\max _{1 \leq i \leq n} \min _{1 \leq j \leq n} r_{i j}=\min _{1 \leq j \leq n} \max _{1 \leq i \leq m} r_{i j} \\
r_{i j^{*}} \leq r_{i^{*} j^{*}} \leq r_{i^{*} j}
\end{gathered}
$$

Definition. A random variable whose values are the strategies of a player is called a mixed strategy of the player 26]

Pure strategy means that the player (agent) will always take the same actions, whereas in mixed strategy the player picks different actions. We can assume player 1 to have mixed strategy $\pi_{1}$ and player 2 to have mixed strategy $\pi_{2}$. This makes the value for the expected rewards for player 1 (2.24) and for player 2 (2.25) [16] [28].

$$
\begin{aligned}
& V_{1}=\pi_{1} R_{1} \pi_{2}^{T} \\
& V_{2}=\pi_{2} R_{2} \pi_{1}^{T}
\end{aligned}
$$

Equations (2.24) and 2.25) can be generalized by (2.26), such that $i$ represents player $i$ and $-i$ represent the other player.

$$
V_{i}=\pi_{i} R_{i} \pi_{-i}^{T}
$$


Let equilibrium situation strategies be described by $\pi_{i}^{*}$ and $\pi_{-i}^{*}$ for player 1 and 2 consecutively, then 2.23 can be rewritten as,

$$
\pi_{i} R_{i} \pi_{-i}^{T *} \leq \pi_{i}^{*} R_{i} \pi_{-i}^{T *} \leq \pi_{i}^{*} R_{i} \pi_{-i}^{T}
$$

This equilibrium situation is defined as the Nash Equilibrium. The Nash Equilibrium is a collection of strategies for all players, such that no player can do better by changing his/her strategy, while all others continue to play their Nash Equilibrium. The Nash Equilibrium will be referred to in the future chapters.

If all other players are playing Nash Equilibrium, the goal of player $i$ then becomes optimizing their strategy $\pi_{i}$ to reach $\pi_{i}^{*}$ and maximize their strategy value 2.26 .

We now solve the problem for a 2-player game. Considering 2-actions game such that $\pi_{i}=\left[p_{1}, p_{2}\right], \pi_{-i}=\left[q_{1}, q_{2}\right]$ and $R_{i}=R, V_{i}$ becomes,

$$
V_{i}=p_{1} q_{1} r_{11}+p_{2} q_{1} r_{21}+p_{1} q_{2} r_{12}+p_{2} q_{2} r_{22}
$$

This can be rewritten as,

$$
V_{i}=q_{1}\left(p_{1} r_{11}+p_{2} r_{21}\right)+q_{2}\left(p_{1} r_{12}+p_{2} r_{22}\right)
$$

Since $q_{1}$ and $q_{2}$ are both positive and $q_{1}+q_{2}=0$, each of the terms in the parenthesis is less than or equal to $V_{i}$ :

$$
\begin{aligned}
& p_{1} r_{11}+p_{2} r_{21} \leq V_{i} \\
& p_{1} r_{12}+p_{2} r_{22} \leq V_{i}
\end{aligned}
$$


The term $V_{i}$ in a 2-player game can then be maximized using linear programming to find $p_{1}$ and $p_{2}:[28$

$$
\begin{array}{r}
r_{11} p_{1}+r_{21} p_{2} \geq V_{i} \\
r_{12} p_{1}+r_{22} p_{2} \geq V_{i} \\
p_{1}+p_{2}=1 \\
p_{k} \geq 0, k=1,2
\end{array}
$$

\subsection{Stochastic Games}

Next we look into stochastic games. Stochastic games are a mix of Markov Decision Processes and Matrix Games [29]. Stochastic games are a multi-stage game that consists of multiple players. Each stage (state) of the game is represented by a matrix game [30].

At each state of the game, the players' payoff is represented by a matrix $R_{i}^{k}$ as seen in (2.16), where $i$ and $k$ denotes the player and the state consecutively. Assuming a 2-player game (players $i$ and $j$ ), the payoff of each player at stage $k$ becomes $r_{i j}^{k}$. The

policy for player $i$ at stage $k$ become $\pi_{i}^{k}$. Moreover, the estimated value of each state described it 2.26 becomes $V_{i}^{k}$. This is very similar to what we have seen in Sec. 2.4 However, we now look at the problem per stage $k$.

We can now use reinforcement learning in stochastic games. We can then represent a $n$-player stochastic game by the tuple $\left(S, A_{1}, \ldots, A_{n}, P, R_{1}, \ldots, R_{n}\right)$. Such that, $S$ is the state space, $A_{i}$ is $i$ th player action space $(i=1, \ldots, n), P$ is the transition probability between states, and $R_{i}$ is the $i$ th player reward function. Therefore, the problem can now be describes as an MDP, such that:

- The agent is in a state at each time-step 
- Player $p$ reward at each state $k$ :

$$
r_{p}=r_{i j}^{k}
$$

- Player $p$ policy at each state $k$ :

$$
\pi_{p}=\pi_{i}^{k}
$$

such that $\pi_{p}(s, a)$ is the probability for player $p$ of selecting action $a$ at state $s$ and $\sum_{a \in A_{p}} \pi_{p}(s, a)=1$

- Value estimate for player $p$ of each state (under policy $\pi$ ):

$$
V_{p}^{\pi}=V_{i}^{k}\left(\pi_{i}^{k}\right)
$$

In stochastic games, the players (agents) are assumed to know the payoff functions at all game states. However, to generalize the problem more, we assume that the players do not know the reward functions, and that they learn more as they play. This is called a repeatable game with partial information. Again, the learning agent needs to maximize the received rewards 31.

For $n$-player game, the collection of all players strategies can be denoted by the tupil $\left(\pi_{1}, \ldots, \pi_{n}\right)$. The expected state value $(V(s))$ can then be the expected discounted future rewards as seen in 2.7). Thus, for a given joint action $\left(a_{1}, \ldots, a_{n}\right)$ we can rewrite (2.5) as:

$$
P\left(s, s^{\prime}\right)=\operatorname{Pr}\left\{s_{t+1}=s^{\prime} \mid s_{t}=s, a_{1}, \ldots, a_{n}\right\}
$$


Then, the expected state-action value for player $i$ becomes,

$$
Q_{i}\left(s, a_{1}, \ldots, a_{n}\right)=\sum_{s^{\prime} \in S} P\left(s, s^{\prime}\right)\left[R_{i}\left(s, a_{1}, \ldots, a_{n}\right)+\gamma V_{i}\left(s^{\prime}\right)\right]
$$

Such that $V_{i}\left(s^{\prime}\right)$ is the expected next state value for player $i$, and is

$$
V_{i}(s)=\sum_{a_{1}, \ldots, a_{n} \in A_{1}, \ldots, A_{n}} Q_{i}\left(s, a_{1}, \ldots, a_{n}\right) \pi_{1}\left(s, a_{1}\right) \ldots \pi_{i}\left(s, a_{i}\right) \ldots \pi_{n}\left(s, a_{n}\right)
$$

Then the Nash Equilibrium happens when all player strategies become $\left(\pi_{1}^{*}, \ldots, \pi_{n}^{*}\right)$. Thus, we define the Nash Equilibrium for the $i$ th player, described in (2.27), as:

$$
\underbrace{V_{i}\left(s, \pi_{1}^{*}, \ldots \pi_{i}^{*}, \ldots, \pi_{n}^{*}\right)}_{V_{i}^{*}(s)} \geq V_{i}\left(s, \pi_{1}^{*}, \ldots \pi_{i}, \ldots, \pi_{n}^{*}\right)
$$

Therefore, the state-action value for player $i$ Nash Equilibrium becomes:

$$
\begin{gathered}
\sum_{a_{1}, \ldots, a_{n} \in A_{1}, \ldots, A_{n}} Q_{i}\left(s, a_{1}, \ldots, a_{n}\right) \pi_{1}^{*}\left(s, a_{1}\right) \ldots \pi_{i}^{*}\left(s, a_{i}\right) \ldots \pi_{n}^{*}\left(s, a_{n}\right) \geq \\
\sum_{a_{1}, \ldots, a_{n} \in A_{1}, \ldots, A_{n}} Q_{i}\left(s, a_{1}, \ldots, a_{n}\right) \pi_{1}^{*}\left(s, a_{1}\right) \ldots \pi_{i}\left(s, a_{i}\right) \ldots \pi_{n}^{*}\left(s, a_{n}\right)
\end{gathered}
$$

We now look again at the 2-player zero-sum game and use the introduced stochastic games definitions. On the assumption that the agent does not know the optimal policy, the state value function for player $i$ following Nash Equilibrium can be calculated as: [16

$$
V_{i}^{*}(s)=\max _{\pi_{i}(s, a)} \min _{a_{-i} \in A_{-i}} \sum_{a_{i} \in A_{i}} Q_{i}^{*}\left(s, a_{i}, a_{-i}\right) \pi_{i}\left(s, a_{i}\right)
$$

This is similar to 2.23 . 


\section{Minimax Q-Learning}

Equation (2.44) expects that the agent knows the Nash Equilibrium action-state value $Q_{i}^{*}$. However, it is not always the case. In multi-player reinforcement learning problems, the expected state-action value function is unknown. Therefore, we can use something similar to the Q-Learning algorithm, which was previously described in Sec. 2.3. The Minimax-Q algorithm can be used to find the optimal strategy to produce the highest value in 2.44. The Minimax algorithm is described in Algorithm $3[16]$.

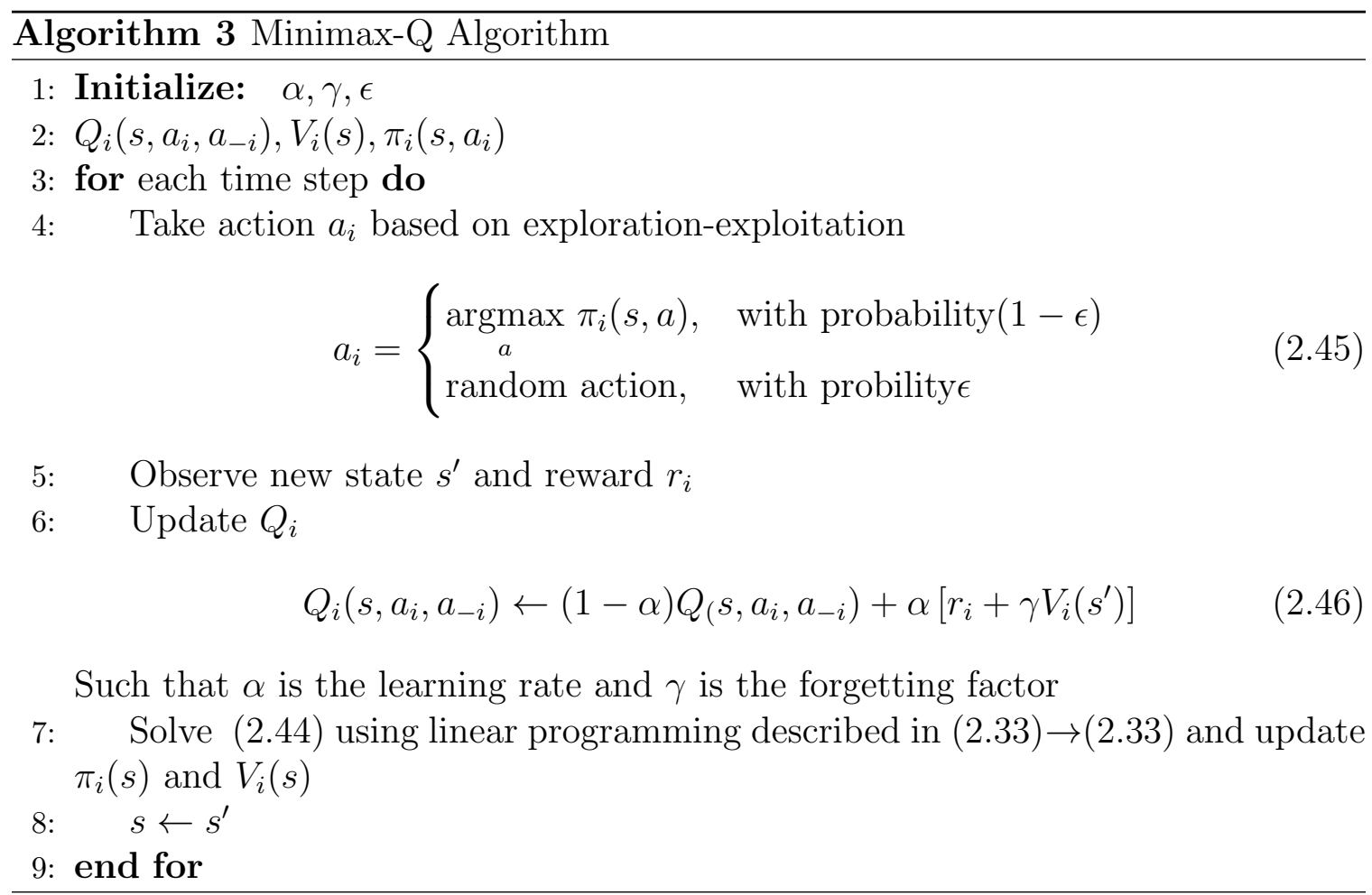

This algorithm is proven to converge if all the different game scenarios are visited often (combination of action and state space). Hence, it is required to add the exploration factor $\epsilon$ as seen in the algorithm [32] 24]. 


\section{WoLF-PHC}

Another possible algorithm that can be used is the WoLF-PHC (Win or Learn Fast

- Policy Hill Climbing algorithm). This algorithm is based on Q-Learning. The WoLF-PHC algorithm is a single algorithm that consists of 2 sub-algorithms. The WoLF part of the algorithm lets the agent learn at 2 different rates; the winning rate $\left(\delta_{w}\right)$ and the losing rate $\left(\delta_{l}\right)$. This means that if the agent is doing well, it should continue with its strategy and improve it. However, if the agent is losing, the agent should change its strategy quickly [33]. Thus, learning faster. The other part of the algorithm, PHC, is a learning algorithm for mixed strategies. The PHC algorithm is proven to converge to a strategy when other players are following a fixed strategy. However, it may not converge when other players are learning [16].

Using the WoLF-PHC algorithm, the agent can guarantee convergence to the Nash Equilibrium. Algorithm 4 describes the WoLF-PHC algorithm for player $i$. 


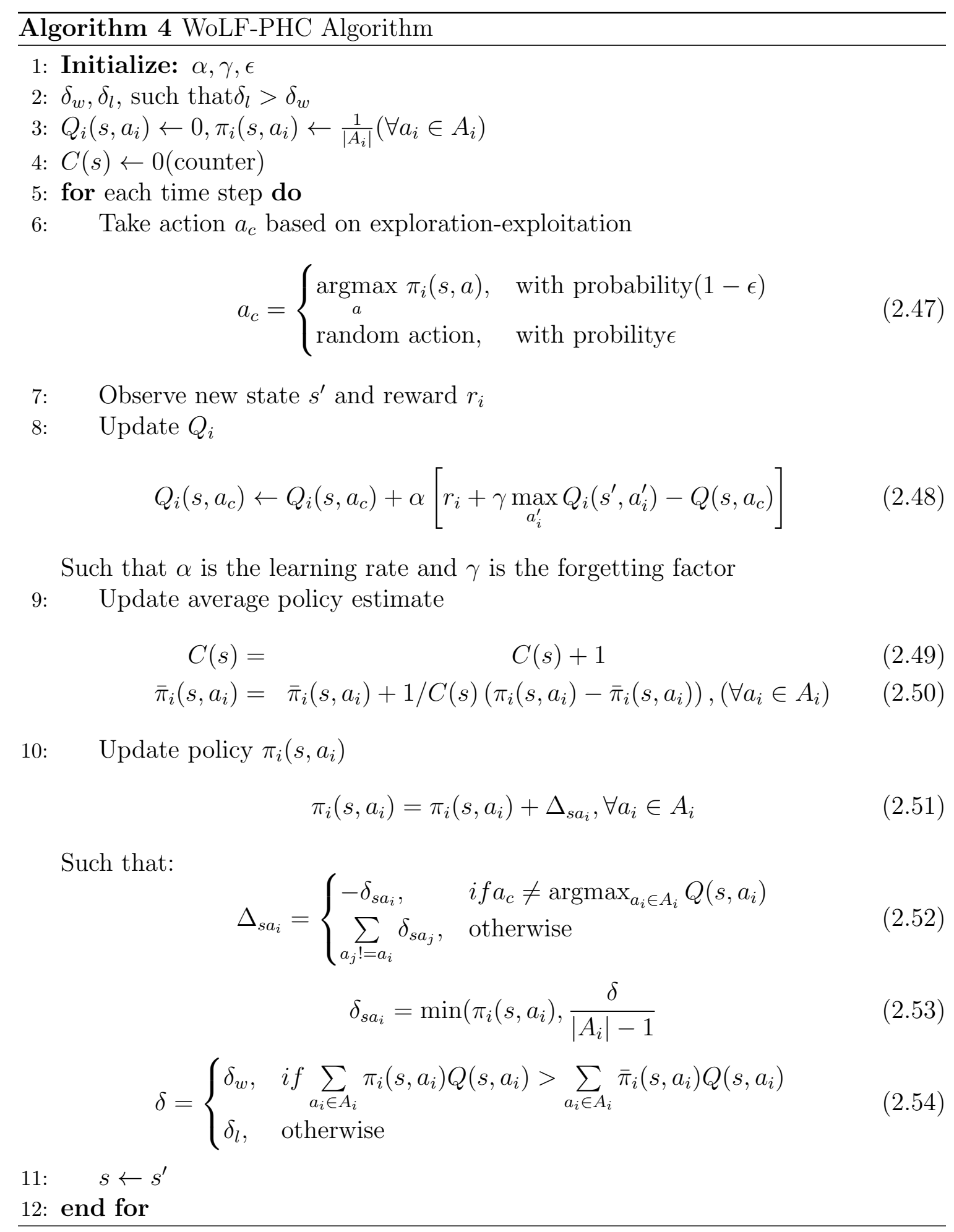




\subsection{Reinforcement Learning in Games}

In literature, RL has been using in many gaming scenarios to allow robots to learn a strategy [34]. Pursuer-evader game is one of the most common games. This is a game where a pursuer is trying to capture an evader [1]. A pursuit-evasion game was investigated in [25]. The authors investigated the use of an algorithm based on hierarchical reinforcement learning and its learning efficiency. They show that using hierarchical reinforcement learning enables the players to learn and "significantly reduces the complexity of the learning task". Givigi and Schwartz in 35 explored a multiple pursuer-evader game. They represented the game as a Markov game and enabled each player to have its own decentralized learning. They show how their agents were able to learn and reached equilibrium points.

As described previously, researchers have combined RL with fuzzy controllers to deal with large space and noisy environments. Schwartz and Desouky in [14] proposed a technique called Q-learning based genetic fuzzy controller. The authors used a fuzzy controller because they investigated the problem in a large continuous state space. The authors show how their players, in the pursuer-evader game, are able to converge their learning using this technique. Schwartz and Desouky in [13] also combined fuzzy logic with RL. The authors tuned parameters of a fuzzy controller using Q-learning in the pursuer-evader game. The parameters are tuned to help the agent take better actions.

The use of RL in digital gaming have been investigated in multiple papers. Galway, Charles and Black in [36] have investigated the ability to improve the temporal difference agent control. The authors investigated the use of a dynamic exploration rate. They investigated in a digital game environment (Pac-Man). Their results show that using a dynamic learning rate, using a genetic algorithm, enhances the 
performance of the agent.

Patel, Carver and Rahimi in [37] looked into the use of RL in computer gaming. They investigated the use of machine learning to enhance the behaviour of bots in games (such as Pac-Man and Counter-Strike). The authors used Q-Learning algorithm to "evolve dynamic intelligent bots". They show how their learning model outperform the static algorithms originally used by the bots. Similarly, work done by the authors in [38] applied RL to a strategy game called StarCraft. They explored the use of RL in managing combat units in real-time (within the game).

RL algorithms have also been used in different board games. Machine learning have also been used in chess game. Work done by the authors in [39] used Minimax Recurrence algorithm (a type of RL) to train an agent to play a flight chess game. They combined RL with supervised learning to produce their learning agent. Their results state that their learning agent is able to compete versus humans and win with a high probability. Moreover, authors in [40] used RL in the game of Othello. They compared the performance of 3 different RL algorithms (Q-learning, TD-learning and Sarsa). This was examined when the opponent followed different strategies.

The prisoner dilemma is a n-player general-sum game. In this game, the players can cooperate or defect. The players choose between cooperating or defecting with the goal of benefiting all players. Vassiliades, Cleanthous and Christodoulou in [41] investigated and illustrated the use of multiagent RL for the prisoner dilemma game. Another general-sum game was investigated by Schwartz and Xiaosongin 42]. The authors used the Lagging Anchor algorithm to demonstrate convergence to Nash equilibrium in pure and mixed strategy matrix games. 


\subsection{Summary}

In summary, this chapter introduced the basis of reinforcement learning, that will be used and referred to in the future chapters, and referred to literature.

First, we introduced Reinforcement Learning. RL described the broader picture of the agent's learning through its interaction with the environment. The agent's interactions result in different states and rewards. The states and rewards have to be designed to describe the problem being tackled using a specific strategy (policy).

Second, we described the Markov Decision Processes. An MDP describes the environment, for a single agent, in terms of a tuple that includes the action space, state space, and state-transition probabilities. MDP also gave a description of how good is a state in comparison with other states. This also lead to defining an optimal policy, which maximizes the agent's rewards.

Third, the Temporal Difference was then described. TD is used to estimate the state values when the state-transition probabilities are unknown. The Q-Learning algorithm was defined and explained as an example of an off-policy TD algorithm.

Fourth, Matrix Games were introduced to describe multiple agent interactions. Matrix Games are used to analyse the learning problem in Game Theory terms. They describe the maximum possible outcome for any game with multiple agents (players). We also defined and illustrated the Nash Equilibrium.

Last, Stochastic Games described the combination of MDP and Matrix Games. This allows us to be able to represent a problem with multiple players and multiple states. We also introduced two learning algorithms; Minimax Q-Learning and WoLFPHC. These algorithms converge to Nash Equilibrium when players are learning. They should also converge to a pure strategy if the opponent is following a fixed strategy. 
All the introduced topics will be used and referred to in creating our learning agent. Future chapters will describe the investigated problem using terminologies from this chapter. They will also contain simulations that demonstrate the performance of different algorithms. 


\section{Chapter 3}

\section{Problem Formulation}

In Chapter 1, we provided a brief description of the problem being investigated. In this chapter we will provide more details of the problem. Details of the game environment will be provided. Then we will describe the theoretical optimal strategies for the players.

\subsection{Problem Description}

Given a game with a single guard $(G)$, single invader $(I)$ and territory $(T)$, the game terminates when the invader reaches the territory or the invader gets captured by the guard. We define a rational invader as follows:

- If it is possible for the invader to reach the territory, a rational invader will reach the territory

- If it is impossible for the invader to reach the territory, a rational invader will get as close as possible to the territory

Based on the game environment, sometimes it is impossible for the invader to reach the territory. We use a rational invader as a benchmark for comparison to our learning invader. 


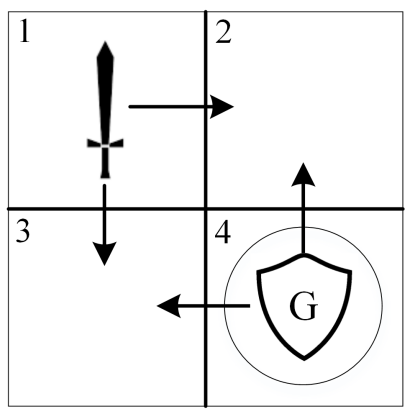

Figure 3.1: $2 \times 2$ Grid Game

For the sake of simplicity, the capturing action happens when the invader is at a specified proximity from the guard defined as dist $t_{\text {capture. }}$. The payoff (result) of the game can be determined as the distance between the invader and the territory at game termination time $\left(t_{f}\right)$ :

$$
\text { payoff }=\sqrt{\left(x_{I}\left(t_{f}\right)-x_{T}\right)^{2}+\left(y_{I}\left(t_{f}\right)-y_{T}\right)^{2}}
$$

$x_{I}\left(t_{f}\right)$ and $y_{I}\left(t_{f}\right)$ represent the invader's coordinates at terminal time. $x_{T}$ and $y_{T}$ represent the territory's coordinates. The goal for the invader is to minimize the payoff and the goal of the guard is to maximize the payoff.

Our objective is to create a learning agent that will be able to minimize its payoff and perform optimally (rationally) in different circumstances.

We will first start with a discretized version of the game (grid world). Then, we will present the differential game version.

\subsubsection{Grid Game}

Figure 3.1 demonstrates a $2 \times 2$ grid game with 2 players, an Invader and a Defender. The invader is represented by the sword in square 1 and the guard is represented by the shield in square 4 . These are the initial position for the players in the game. The 
territory is also in square 4 (bottom right box). This stochastic game is the simplest form of the guarding a territory game. This game example was investigated in [4]. We define the players' rewards as:

$$
\begin{aligned}
& r_{G}= \begin{cases}1, & \text { Guard captures invader } \\
-10, & \text { Invader reaches territory }\end{cases} \\
& r_{I}= \begin{cases}-1, & \text { Guard captures invader } \\
10, & \text { Invader reaches territory }\end{cases}
\end{aligned}
$$

In other words, if the invader reaches the territory, the guard will be penalized and the invader will be awarded. Alternatively, if the guard captures the invader, the guard is awarded and the invader is penalized. No rewards are received in nonterminal states. Capturing happens when both the guard and the invader are in the same square (i.e.dist capture $=0$ ). The action spaces for both players are defined by the arrows in Figure 3.1, such that:

$$
\begin{aligned}
A_{\text {invader }} & =[\text { down, right }] \\
A_{\text {guard }} & =[\text { up, left }]
\end{aligned}
$$

Each player can only move to the adjacent square. We can then deduce all the possible game states as seen in Figure 3.2 and Figure 3.3 . We can then deduce the state-transition probabilities $P\left(a_{I}, a_{G}\right)$ as seen in Figure 3.4. Such that, $a_{I}$ and $a_{G}$ are the invader and guard actions respectively.

If the game is in non-terminal state $s_{1}$ or $s_{2}$, the invader can reach the territory 


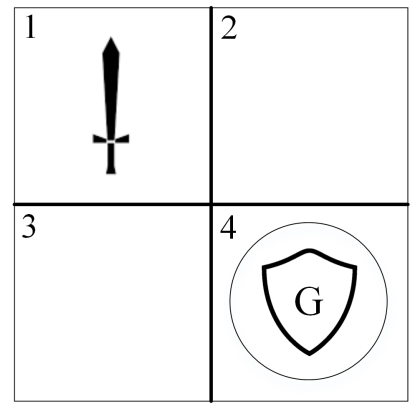

(a) $s_{0}$

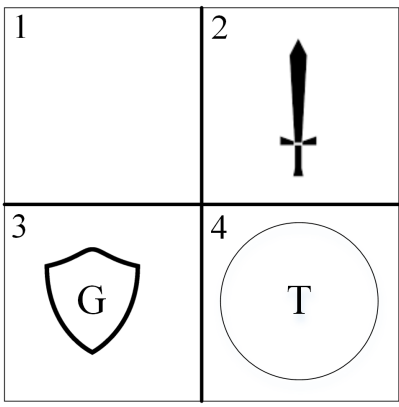

(b) $s_{1}$

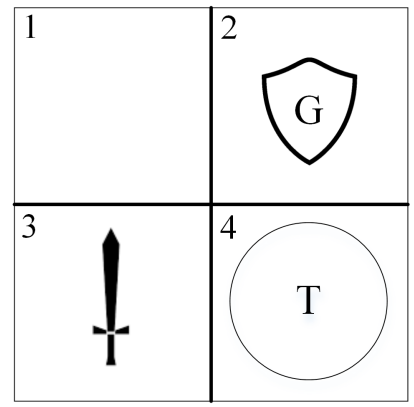

(c) $s_{2}$

Figure 3.2: Grid Game - Non-Terminal States

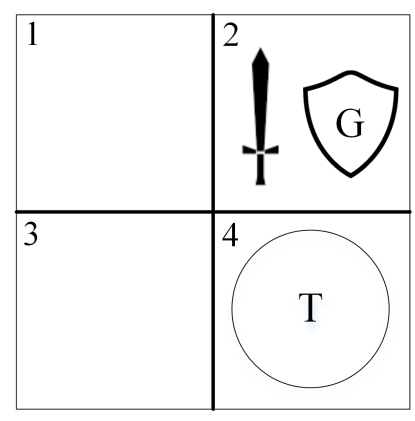

(a) $s_{3}$

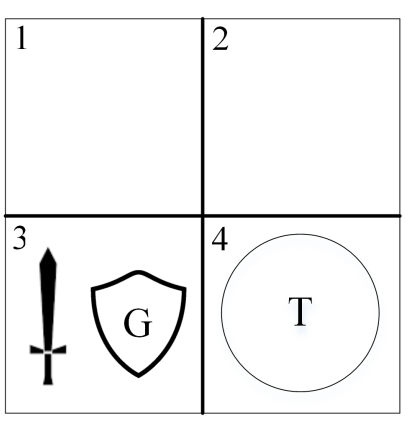

(b) $s_{4}$

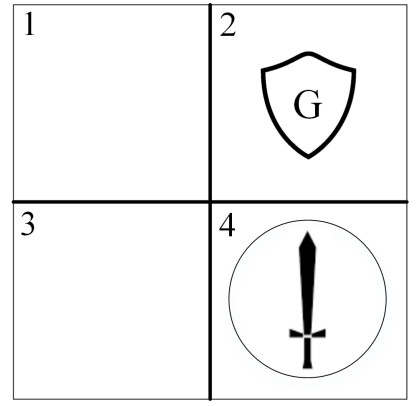

(c) $s_{5}$

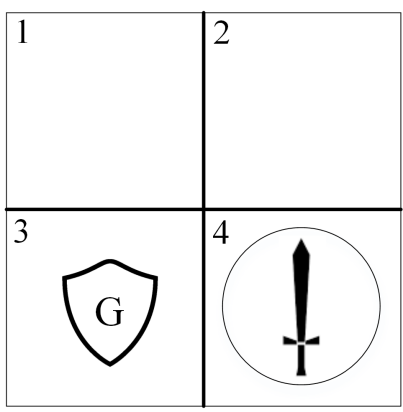

(d) $s_{6}$

Figure 3.3: Grid Game - Terminal States 


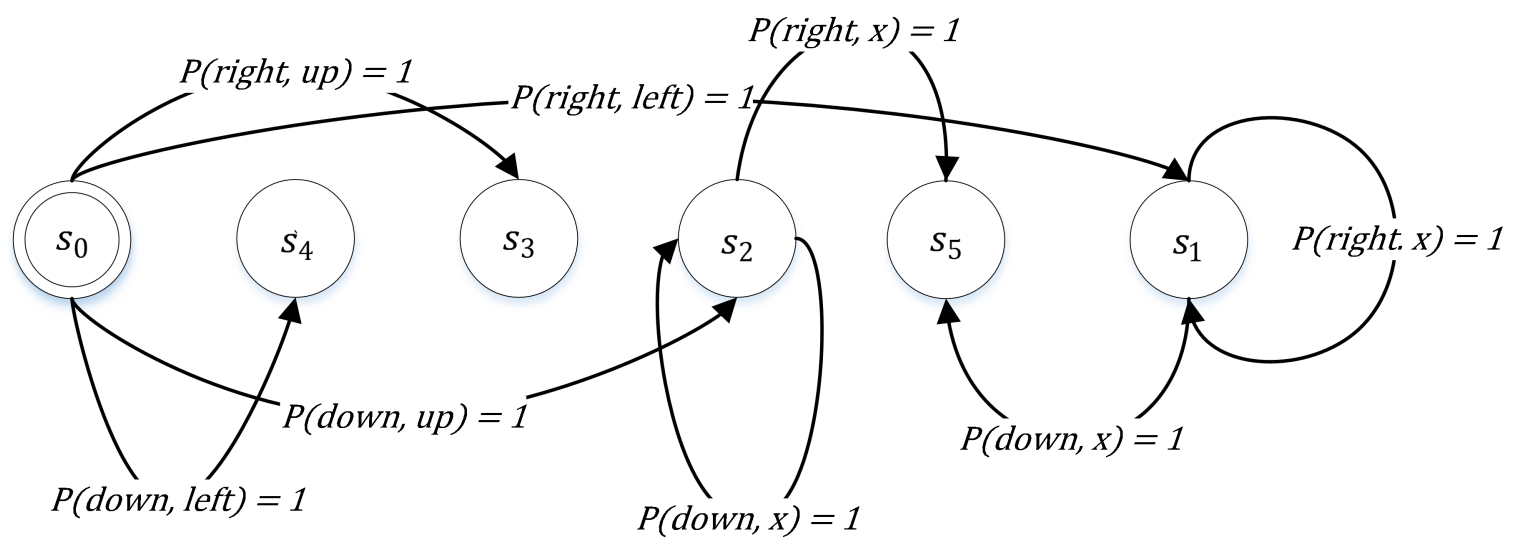

Figure 3.4: State Transition Probabilities

without being stopped by the guard. The output of the game depends on the first action. If both players move to the same square, the invader will lose. Otherwise, the invader will win. Thus, we now know the following about the guard's state value function: $V_{G}\left(s_{2}\right)=V_{G}\left(s_{3}\right)=-10$. We also know the state-action function at initial state, such that $Q_{G}\left(s_{0}, a_{I}=\right.$ right, $\left.a_{G}=u p\right)=Q_{G}\left(s_{0}, a_{I}=\right.$ down, $a_{G}=$ right $)=1$. This becomes the Nash Equilibrium action-state value $Q_{G}^{*}$ at this state.

With the information we know, linear programming can be used to calculate the states values and the players' optimal policies. This will result in the Nash Equilibrium policy for the invader to move down or right with a probability of 0.5 at state $s_{0}$, also the Nash Equilibrium policy for the guard at the same state becomes moving up or left with a probability of 0.5 .

By this, we have a $2 \times 2$ grid game with 2 players that contains action space of 7 different states, 2 actions within each player's action space. As the size of the game increases, the number of states would increase, as well as, the admissible state-action pairs. Increasing the action space would also increase the number of the admissible state-action pairs. 


\subsection{Guarding a Territory in Differential Games}

We increase the complexity of our game by describing it as a differential game occurring in continuous time. Working in continuous time, any player $j$ (invader or guard) will have the following equations of motion:

$$
\left[\begin{array}{c}
\dot{x}_{j} \\
\dot{y}_{j} \\
\dot{\varphi}_{j}
\end{array}\right]=\left[\begin{array}{c}
\cos \varphi_{j} \\
\sin \varphi_{j} \\
0
\end{array}\right] v_{j}+\left[\begin{array}{l}
0 \\
0 \\
1
\end{array}\right] \omega_{j}
$$

$$
\operatorname{such} \text { that }\left\{\begin{array}{l}
x: x-\text { coordinates, } \\
y: y-\text { coordinates, } \\
\varphi: \text { robot orientation (heading), } \\
v: \text { driving velocity, and } \\
\omega: \text { angular speed }
\end{array}\right.
$$

These equations of motion are the same kinematics of a cart robot with non-holonomic constraints 43 .

Our game occurs in a bounded space. Both the guard and the invader have to stay within the borders of the game-space. Hitting the border will make the player stay in the same position. This constraint is enforced such that the game will quickly come to completion.

In this thesis we will make both players have the same angular speed, but the linear speed of the guard will be 20 percent faster than that of the invader. The speed of the guard was chosen to be 20 percent faster so the game will not terminate too quickly. This is also due to the bounded game size. Moreover, if the guard was 


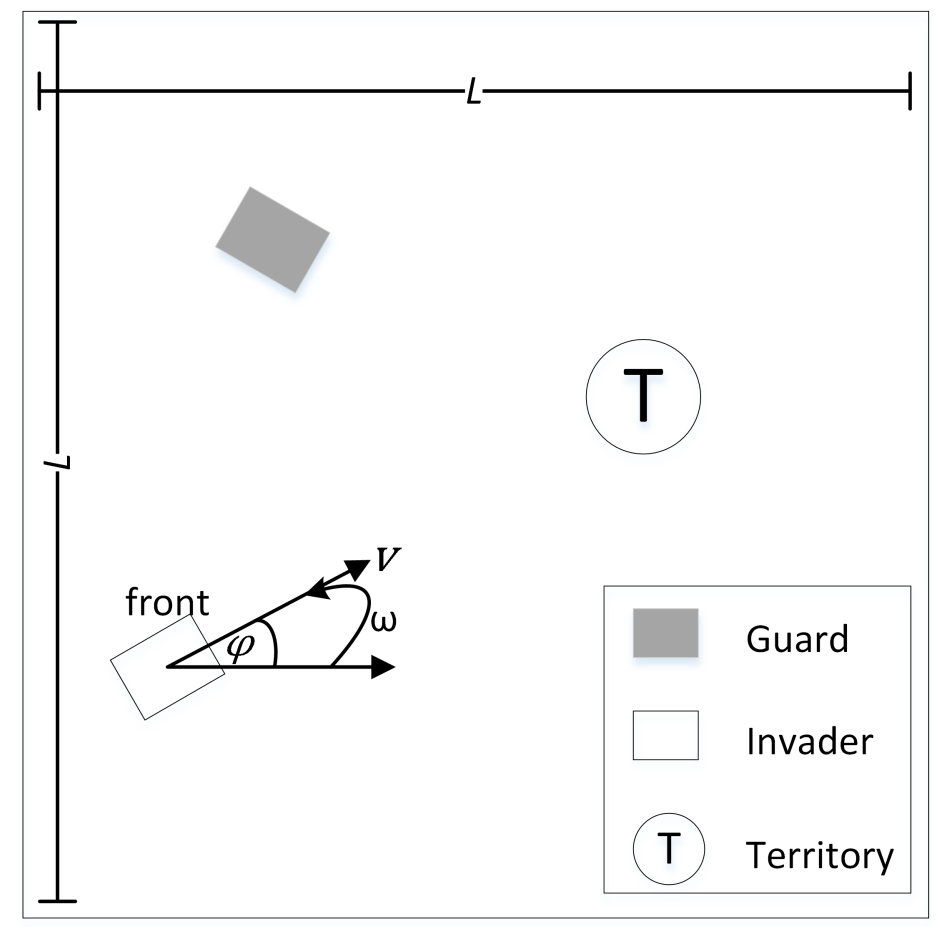

Figure 3.5: Game Environment

faster than 20 percent, it would not have been able to take steep turns.

Figure 3.5 displays the game layout. Only the invader's kinematics are labelled. Please note that the dimensions of the robot does not depict what is used for simulation, but it is only for illustrative purposes. The term $L$ is the length of the game's bounded space ( $L=30$ unitspace).

\subsection{Guard's Strategy}

\subsubsection{Guard's Optimal Strategy}

Different guarding strategies were considered to play against the learning invader. On the assumption that the invader can be captured, [1] describes the guard's optimal 


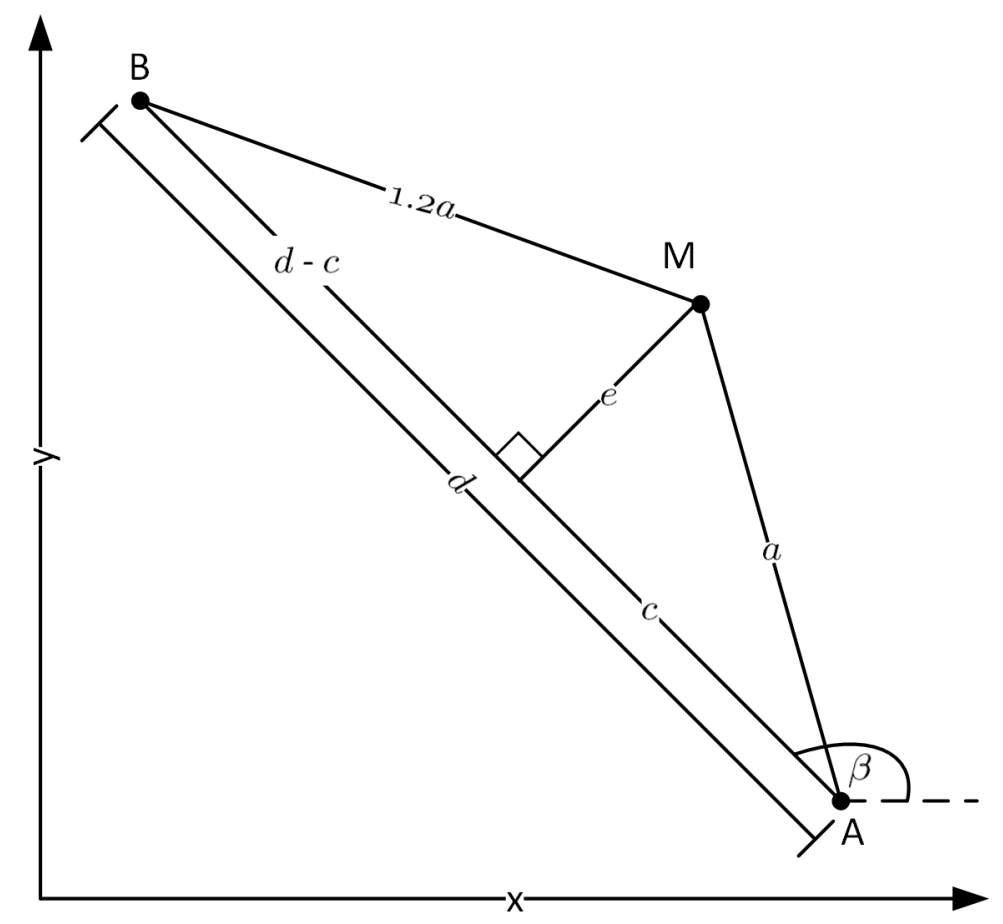

Figure 3.6: Reachable region geometry

policy as follows: if both the guard and the invader have equal kinematics, the perpendicular bisector line between the two players (at each time step) will indicate the region that the invader can reach before being captured by the guard. This is the region on the invader's side of the line. Once the guard knows this line, it should move towards the point closest to the territory that lies on this line. This point is called the closest interception point (closest to the territory). The guard has to calculate this point at each time step. The only optimal strategy against the guard's optimal strategy is that the invader has to also aim at the closest interception point. This will allow the invader to get as close as possible to the territory, and hence achieve the minimum payoff possible. This is described as the Nash Equilibrium for both players.

Since in the investigated case the guard is 20 percent faster than the invader, the line that describes the region that can be reached by the invader is no longer the 
perpendicular bisector.

According to Figure 3.6, the invader is at point ' $\mathrm{A}$ ' and the guard is at point ' $\mathrm{B}$ ' (separated by distance $d$ ) and that the speed of the guard is 20 percent faster than that of the invader, the invader can reach any point ' $\mathrm{M}$ ' before being captured by the guard; such that the distance between ' $\mathrm{A}$ ' and ' $\mathrm{M}$ ' is ' $a$ ' and the distance between ' $\mathrm{B}$ ' and ' $\mathrm{M}$ ' is ' $1.2 a$ '. The set of points that can satisfy the condition for ' $\mathrm{M}$ ' are calculated as follows,

$$
\begin{gathered}
e^{2}+c^{2}=a^{2} \\
1.44 e^{2}+1.44 c^{2}=1.44 a^{2} \\
e^{2}+(d-c)^{2}=1.2^{2} a^{2} \\
e^{2}+d^{2}-2 d c+c^{2}=1.44 a^{2} \\
0.44 c^{2}+2 d c+\left(0.44 e^{2}-d^{2}\right)=0
\end{gathered}
$$

For $e \in \mathbb{R}, c$ can be found as:

$$
c=\frac{-(2 d)^{2} \pm \sqrt{2 d-4 \times 0.44 \times\left(0.44 e^{2}-d^{2}\right)}}{2 \times 0.44}
$$

This function (3.5) is represented in the global reference frame. It will have to be rotated by angle $\beta$ then traversed around point 'A'. This can be done using the rotation matrix $R$ (shown below), then adding vector point 'A' coordinates vector.

$$
R=\left[\begin{array}{cc}
\cos \beta & -\sin \beta \\
\sin \beta & \cos \beta
\end{array}\right]
$$

Once the set of points is deduced and on the assumption that capturing is possible, 


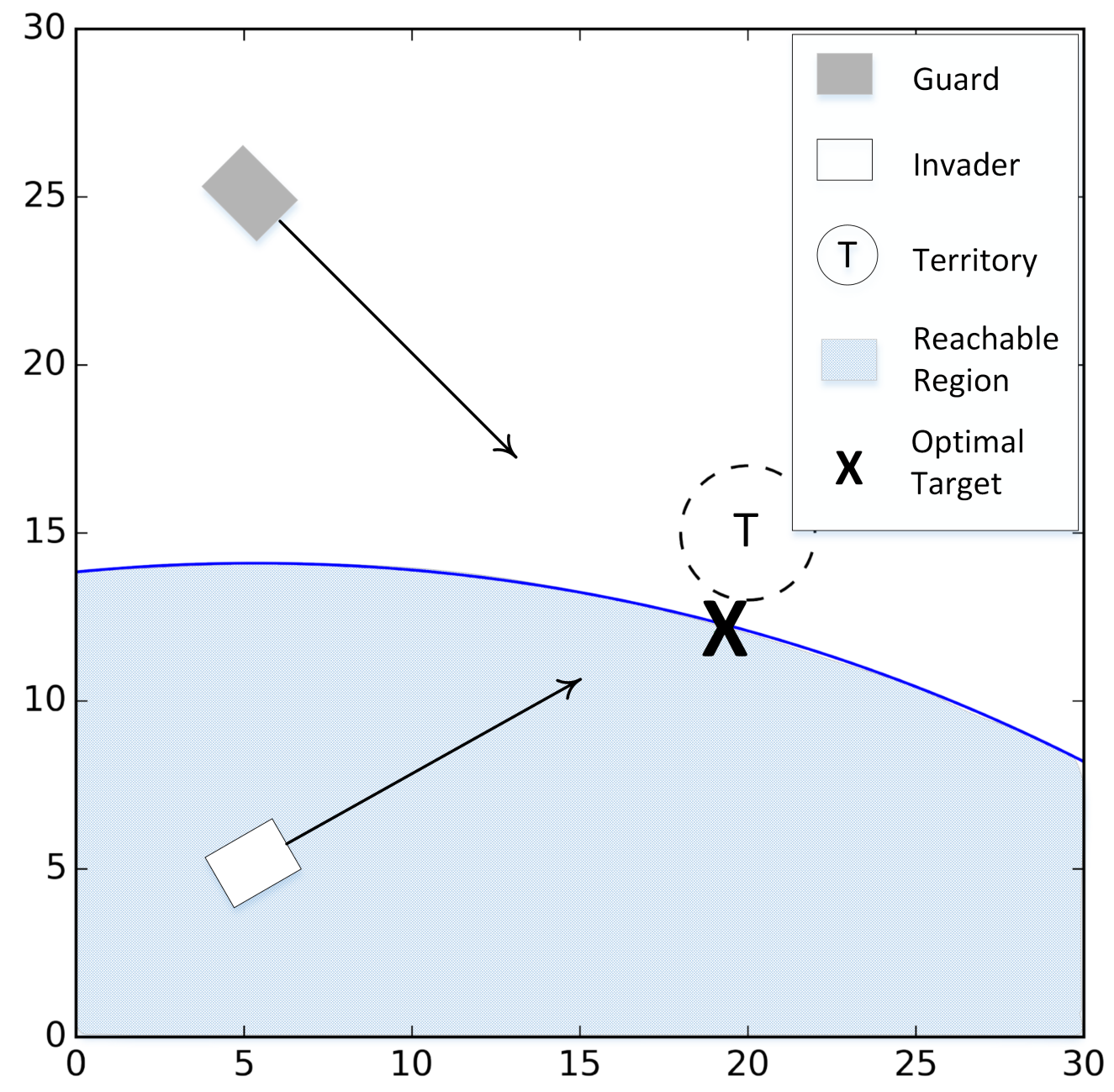

Figure 3.7: Guard's Optimal Strategy - Nash Equilibrium

the guard's optimal policy against any invader is to target the closest interception point. If the invader targets the same point, then the invader is following the Nash Equilibrium strategy for an optimal guard. Figure 3.7 illustrates the strategies.

\subsubsection{Irrational Guard Strategy}

Another guard algorithm used in our thesis is targeting the invader's current position. This strategy performs worse than the optimal strategy, because it does not try to 
intercept the invader. We call this strategy the irrational guard approach.

In this strategy, the guard will ignore the position of the territory. It will aim at decreasing the distance between the guard and the invader. The invader's or guard's heading is also not considered. Thus, the guard will not be performing optimally and this will give a chance for the invader to reach the territory in some cases.

If both players are playing rationally, then they will reach the Nash Equilibrium and neither of them can do any better. However, if one of them is not being rational, the other opponent may be able to do better.

\subsection{Estimating the Game State}

Since the current game runs in continuous time with a bounded space, it is possible to discretize the entire game space. However, this will require a very large space of memory to be implemented on a simple device. The same goes for the action space. Moreover, this discretization will cause a slow learning, since the agent will have a large amount of tables to fill and update. Discretization will also be computationally expensive, and hence discretizing the spaces is less favorable.

A small step size is required to discretize the space and model continuous time. Discretizing the game space for a single player, in our current game environment with 0.01 unit step size, will produce $9 \times 10^{6}$ entries $\left(30 \times 30 / 0.01^{2}\right)$. This will be a massive table that does not consider the other player's position. This will produce a total of $81 \times 10^{12}$ states for both players. Therefore, the use of a fuzzy logic controller is proposed to estimate the current system state. A fuzzy logic estimator with a reinforcement learning algorithm is used to produce our learning agent. Other than estimating the state, the fuzzy logic controller helps in dealing with noise signals. 


\section{Chapter 4}

\section{The Control System}

This chapter will describe the control system behind our learning agent in detail. Fuzzy logic is used as the basis for building our control system. A detailed description of our fuzzy controller is provided. Finally, we show how we can combine the fuzzy controller with RL in two different learning algorithms; Fuzzy Q-Learning and Fuzzy Actor Critic Learning.

\subsection{Fuzzy Logic Introduction}

A fuzzy inference engine is a universal estimator. It can be used to describe an environment when precise information is hard to obtain. Fuzzy systems are rule-based systems. A rule-based system will map an input $x(x \in X)$ to an output through using a set of "IF... THEN" rules that relate the input to the output, such that $X$ is the universe of discourse.

The IF... THEN rules represent linguistic rules that can be converted into a mathematical expression by using Membership Functions [44]. For example,

rule: IF wind is high, THEN temperature is cold 


\subsubsection{Fuzzy Rules}

A more general example of 4.1 would be [16]:

R: If $x$ is $A$, THEN $y$ is $B$

Such that $A$ and $B$ are the fuzzy sets. " $x$ is $A$ " is a Fuzzy Preposition $\left(F P_{1}\right)$ that

is the observation (premise), and " $y$ is $B$ " is a Fuzzy Preposition $\left(F P_{2}\right)$ that is the conclusion. The membership function is a characteristic or an indicator function. The membership functions have values between 0 and 1 . They map the input, for a specific rule, to a degree between 0 and 1 . For fuzzy set $A$, the membership function (degree) is denoted by $\mu_{A}(x)$. Different functions can be used to express the membership function. Such as, trapezoidal, Gaussian, triangular, etc [45].

The union and intersection between fuzzy set $A_{1}$ and $A_{2}$ is represented by (4.3) and (4.4). Where $\dot{+}$ is called a s-norm operator and $\star$ is called a t-norm operator.

$$
\begin{gathered}
\mu_{A_{1} \cup A_{2}}(x)=\max \left[\mu_{A_{1}}(x), \mu_{A_{2}}(x)\right]=\mu_{\left.A_{1}\right)} \dot{+} \mu_{A_{2}} \\
\mu_{A_{1} \cap A_{2}}(x)=\min \left[\mu_{A_{1}}(x), \mu_{A_{2}}(x)\right]=\mu_{\left.A_{1}\right)} \star \mu_{A_{2}}
\end{gathered}
$$

Another interpretation of fuzzy IF . . THEN rules is Madmani Implications. It relates 2 FPs with a single membership function:

$$
\mu_{Q}(x, y)=\min \left[\mu_{F P_{1}}, \mu_{F P_{2}}\right]
$$

The canonical form for the fuzzy IF-Then rules (for any rule $l$ ) can be described as:

$$
R^{l}: \boldsymbol{I F}: x_{1} \text { is } A_{1}^{l} A N D x_{2}^{l} \text { is } A_{2}^{l} A N D \ldots A N D x_{k}^{l} \text { is } A_{k}^{l} \text {, THEN: } y=B^{l}
$$


Where $A_{i}^{l}(i=1, \ldots, k)$ is a fuzzy set in $U_{i}\left(U=U_{1} \times U_{2} \times \ldots \times U_{k} \subset R^{k}\right)$ and $B^{l}$ is a fuzzy set in $V(V \subset R)$ 46].

Takagi and Sugeno in [47] describe a fuzzy system that maps the fuzzy inputs to a linear function. They define the fuzzy rules as:

$$
R: \text { IF } f\left(x_{1} \text { is } A_{1}, \ldots, x_{k} \text { is } A_{k}\right) \text {, THEN } y=g\left(x_{1}, \ldots, x_{k}\right)
$$

Such that, $x_{1}, \ldots, x_{k}$ are the fuzzy inputs, $A_{1}, \ldots, A_{k}$ are the fuzzy sets, and $f($.$) is$ the premise function. $g($.$) is our conclusion function. Takagi and Sugeno developed$ a linear function to describe the conclusion function:

$$
\begin{gathered}
R: \boldsymbol{I F}: x_{1} \text { is } A_{1} A N D x_{2} \text { is } A_{2} A N D \ldots A N D x_{k} \text { is } A_{k}, \\
\text { THEN: } y=K_{0}+K_{1} x_{1}+\ldots+K_{k} x_{k}
\end{gathered}
$$

Where $K_{i}(i=0, \ldots, k)$ is the conclusion parameter. We will generalize (4.8) for any rule $l$ to become $[16]$ :

$$
\begin{gathered}
R^{l}: \boldsymbol{I F}: x_{1} \text { is } A_{1}^{l} A N D x_{2} \text { is } A_{2}^{l} A N D \ldots x_{k} \text { is } A_{k}^{l}, \\
\text { THEN }: y^{l}=K_{0}^{l}+K_{1}^{l} x_{1}+\ldots+K_{k}^{l} x_{k}
\end{gathered}
$$

Each rule $R^{l}$ produces a single output $y^{l}$. The rules can be combined using a fuzzy inference engine to produce a single rule. In other words, inference engines are used to combine the IF-THEN rules [46] to map fuzzy set $A^{\prime} \subset U$ to fuzzy set $B \subset V$. Hence, representing the combination of rules as a single rule.

The product inference engine is one type of inference engine. It consists of individual rule base inference, Madmani's product implication, algebraic product for all t-norm operators and $\max$ for s-norms. The product inference engine output then 


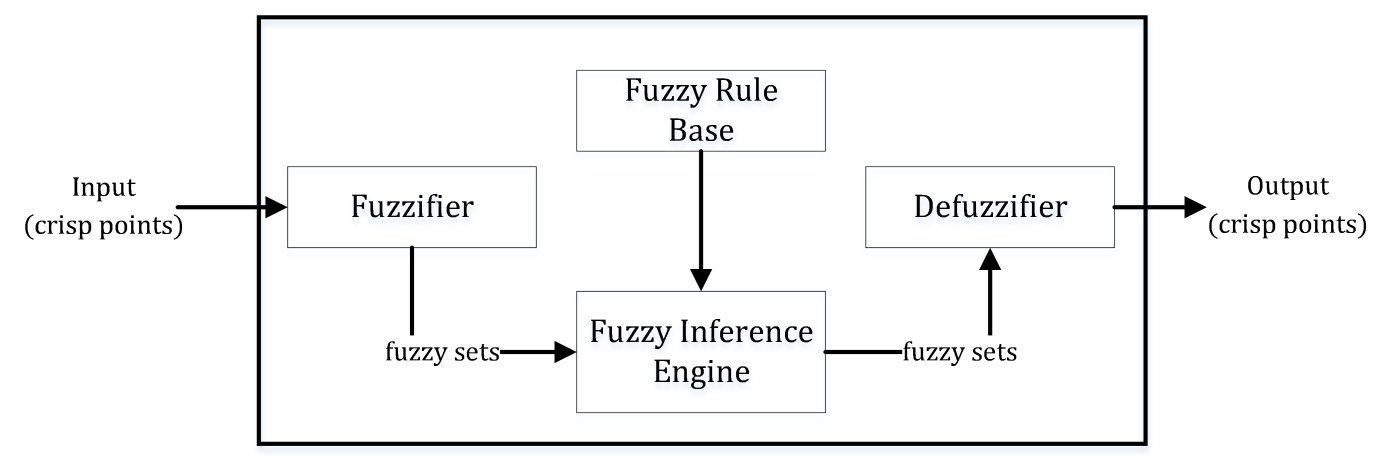

Figure 4.1: Fuzzy System

becomes 46:

$$
\mu_{B^{\prime}}(y)=\max _{l=1}^{M}\left[\sup _{\mathrm{x} \subset U}\left(\mu_{A^{\prime}}(\mathrm{x}) \prod_{i=1}^{n} \mu_{A_{i}^{l}}\left(x_{i}\right) \mu_{B^{l}}(y)\right)\right]
$$

\subsubsection{Fuzzy System}

One can combine and use the fuzzy rules properties to create a fuzzy system, as seen in Figure 4.1. A fuzzifier maps a real-value (crisp point) to a fuzzy set. This is done by calculating the membership value for each input based on the rules that apply to this input. In this thesis, we use a Singleton fuzzifier (4.11) to map a real-value $x^{*}$ to the fuzzy set $A$.

$$
\mu_{A}(x)= \begin{cases}1 & \text { if } x=x^{*} \\ 0 & \text { otherwise }\end{cases}
$$

The Defuzzifier maps a fuzzy set to a real-value. Many defuzzifiers exist in the literature. In our thesis, we use the weighted average defuzzification method for its computational simplicity [46]. The output of this defuzzification can be represented as [16]:

$$
y^{*}=\frac{\sum_{l=1}^{N}\left(\prod_{i=1}^{n} \mu_{i}^{l}\left(x_{i}\right) w_{l}\right)}{\sum_{l=1}^{N}\left(\prod_{i=1}^{n} \mu_{i}^{l}\left(x_{i}\right)\right)}
$$


Such that: $w_{l}$ is the center of the l's fuzzy rule, $N$ is the number of rules, $n$ is the number of inputs, and $\mu_{i}^{l}$ is the membership degree function at rule $l$ for input $i$.

\subsection{The Fuzzy Controller}

We created our control system by using a singleton fuzzifier, a product inference engine and weighted average defuzzification. The output of our fuzzy system becomes:

$$
\begin{gathered}
u=\frac{\sum_{l=1}^{N}\left(\prod_{i=1}^{n} \mu_{i}^{l}\left(x_{i}\right) c_{l}\right)}{\sum_{l=1}^{N}\left(\prod_{k=1}^{n} \mu_{k}^{l}\left(x_{k}\right)\right)}=\sum_{l=1}^{N} \phi^{l} c_{l} \\
\phi^{l}=\frac{\prod_{i=1}^{n} \mu_{i}^{l}\left(x_{i}\right)}{\sum_{j=1}^{N}\left(\prod_{k=1}^{n} \mu_{k}^{j}\left(x_{k}\right)\right)}
\end{gathered}
$$

$c_{l}$ is a constant describing the fuzzy set. Another benefit of having a fuzzy system is that it will also help us in dealing with noisy input.

Our proposed invader's controller was designed such that:

1. It will collect information from the game (inputs)

2. The fuzzy system will estimate the game's state

3. The fuzzy system with Reinforcement Learning will guide the invader

\section{Controller Inputs}

To select our inputs, we proposed using variables that will define the positions of the players and the territory. 
The first input was chosen to describe the territory position with respect to the invader. The angle $(\theta)$ between the global x-axis and the invader's line of sight towards the territory was used, as shown in Figure 4.2. Such that $\theta \in[0,2 \pi]$. The input $\theta$ is then divided into 9 fuzzy sets as follows:

- ZE: $\theta$ is near 0 (Zero)

- SS: $\theta$ is near $\pi / 4$ (Small Small)

- SM: $\theta$ is near $\pi / 2$ (Small Medium)

- SL: $\theta$ is near $3 \pi / 4$ (Small Large)

- MS: $\theta$ is near $\pi$ (Medium Small)

- MM: $\theta$ is near $5 \pi / 4$ (Medium Medium)

- ML: $\theta$ is near $6 \pi / 4$ (Medium Large)

- LS: $\theta$ is near $7 \pi / 4$ (Large Small)

- LM: $\theta$ is near $2 \pi$ (Large Medium)

Previous research done by [5] used less fuzzy sets to describe the angle in similar game. However, we found this number of sets (9 fuzzy sets) provided better performance through simulations and testing. Our tests showed that the invader performed better using 9 fuzzy sets than if the input was divided to 6,7 or 8 fuzzy sets. Increasing the number of fuzzy sets will increase the number of rules without high significance.

The second and third inputs were used to relate the current guard's position with respect to the invader. Those inputs were selected to be the Manhattan distance between the invader and the guard positions. They are represented by two components $d_{1}$ and $d_{2}$ as shown in Figure 4.3. Such that $d_{1}$ and $d_{2} \in[-L, L]$. Manhattan 


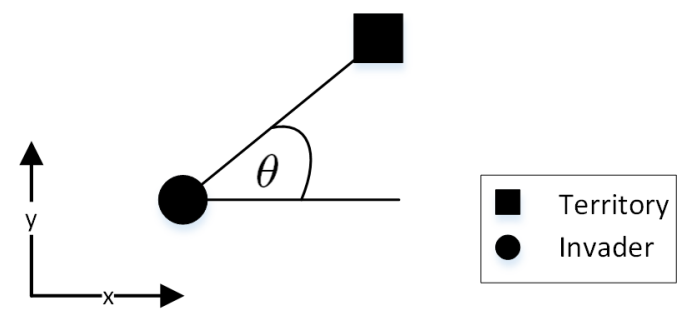

Figure 4.2: angle $\theta$ between invader and territory

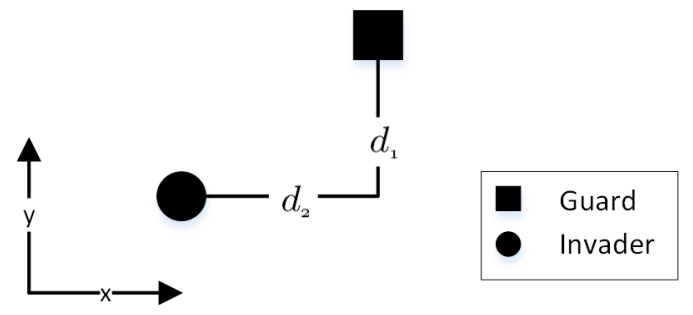

Figure 4.3: Manhattan distance between guard and invader

distance was used instead of the Euclidean distance because it contains more information about the relative position between the guard and the invader positions. Also, simulations showed faster learning using Manhattan distance rather than using both Euclidean distance and the angle between the invader and guard.

For both $d_{1}$ and $d_{2}$, the following 5 fuzzy sets are created $(i \in 1,2)$ :

- LN: $d_{i}$ near -20 (large negative)

- $\mathrm{SN}: d_{i}$ near -10 (small negative)

- ZE: $d_{i}$ near 0 (zero)

- SP: $d_{i}$ near 10 (small positive)

- LP: $d_{i}$ near 20 (large positive)

The membership degree function between the rules was chosen as a triangular function with no adaptation for all the inputs. This no adaptation means that the 


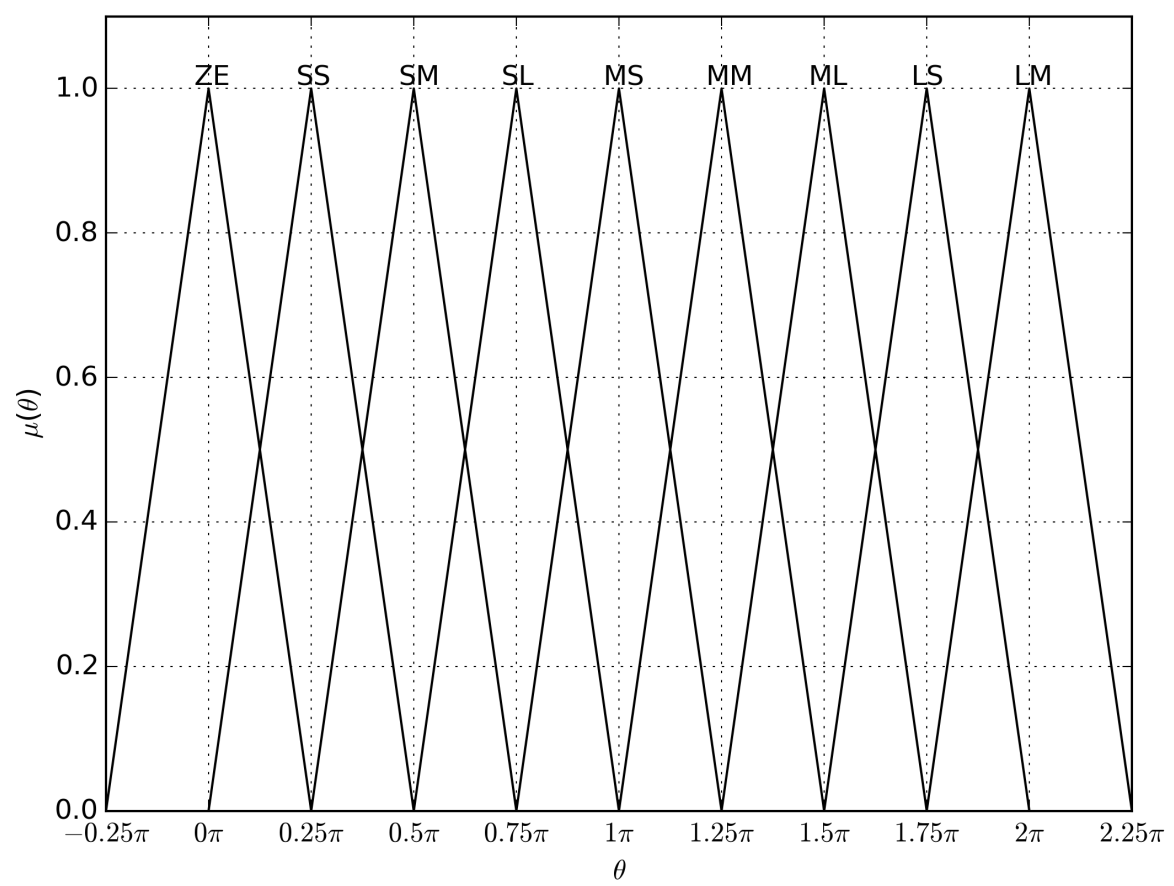

Figure 4.4: Membership function for the angle input

center of the membership functions does not change. Figure 4.4 and Figure 4.5 display the membership functions for the angle $\theta$ and the Manhattan distance inputs.

Other control system inputs were also tested, such as using the Manhattan distance between the invader and the territory; however, this would have required 625 rules and would be computationally complex. In summary, using the previously mentioned inputs $\left(\theta, d_{1}\right.$ and $\left.d_{2}\right)$ creates 225 different combinations, which are 225 rules for our fuzzy system.

\subsection{Fuzzy Q-Learning (FQL)}

Given the current state $\bar{x}_{t}$ and using the fuzzy controller described in 4.1.2, one can compute the output $U_{t}$ as:

$$
U_{t}\left(\bar{x}_{t}\right)=\sum_{l=1}^{N} \phi_{t}^{l} a_{t}^{l}
$$




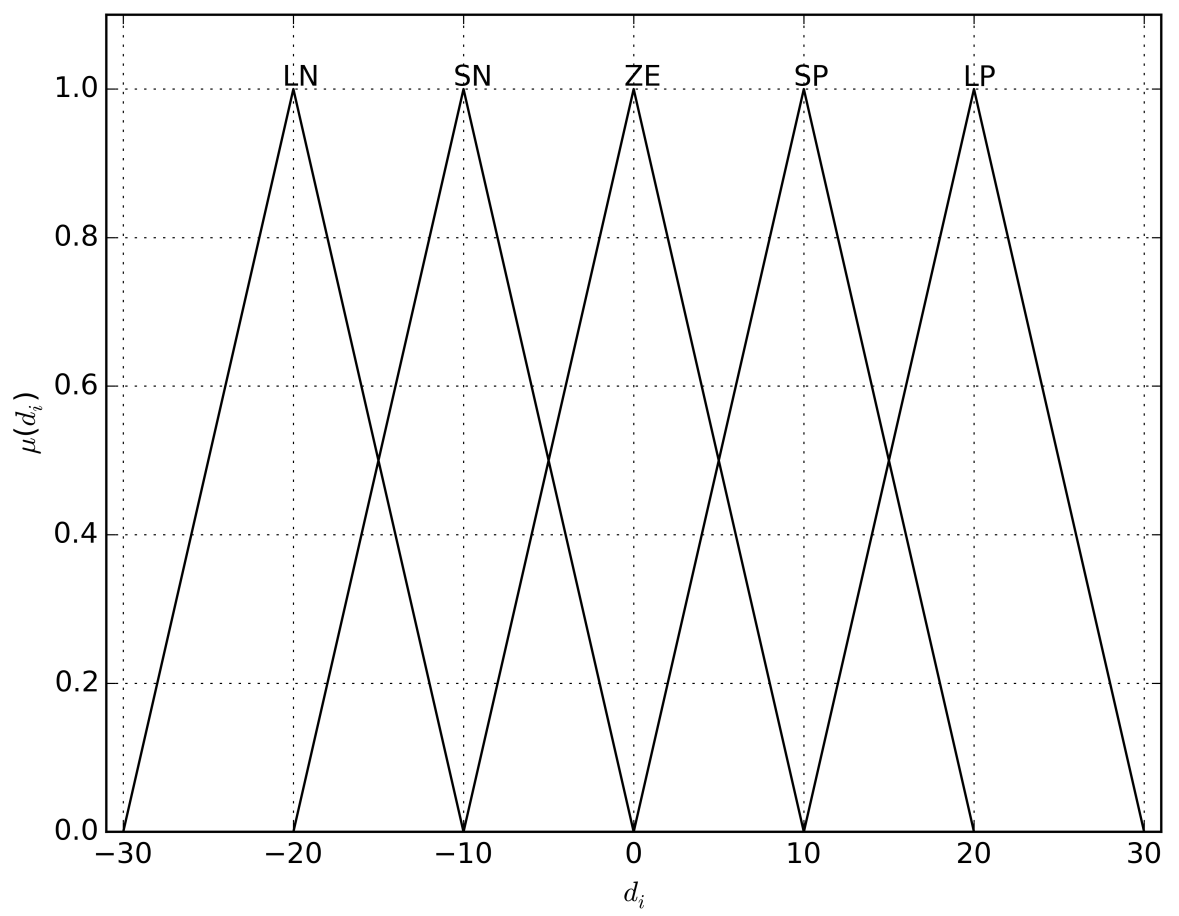

Figure 4.5: Membership function for Manhattan distance input

Where $\phi^{l}$ is same as in (4.14), $\bar{x}=\left[x_{1}, x_{2}, x_{3}\right]=\left[\theta, d_{1}, d_{2}\right], n$ is the number of inputs $(n=3)$ and $N$ is the number of rules $(N=225)$.

Where $a_{t}^{l}$ is the constant describing the centre of the fuzzy set, which in our case is the action selected at time $t$ for rule $l$ based on the Q-table (will be described afterwards). Also, action $a$ belongs to an action set $A$. The action was selected to describe different possible robot headings. The action set was chosen as:

$$
A=\{-3 \pi / 4,-\pi / 2,-\pi / 4,0, \pi / 4, \pi / 2,3 \pi / 4, \pi\}
$$

After taking any action, the action is evaluated. Based on our evaluation a value is added to the Q-table that indicates the reward associated with the action taken.. The Q-table contains a value for each action in the action set for each of the rules $(\operatorname{size}=225 \times 8)$. For each rule, the action with a higher value is more favourable; 
however, a random selection factor was added to create exploration. The values for each of the actions are updated and adapted based on the learning algorithm. The value for the current state is represented by capital $Q$, and the Q-table is represented by lower-case $q$. One can compute $Q$ for the current state as,

$$
Q\left(\bar{x}_{t}\right)=\sum_{l=1}^{N} \phi_{t}^{l} q_{t}\left(l, a_{t}^{l}\right)
$$

The maximum possible value is represented as $Q^{*}$ and computed as,

$$
Q^{*}\left(\bar{x}_{t}\right)=\sum_{l=1}^{N} \phi_{t}^{l} \max _{a \in A} q_{t}\left(l, a_{t}^{l}\right)
$$

One computes the future temporal difference as,

$$
\Delta_{t+1}=r_{t+1}+\gamma Q^{*}\left(\bar{x}_{t+1}\right)-Q\left(\bar{x}_{t}\right)
$$

Where $\gamma$ is the forgetting factor and it focuses on the expected future rewards (as seen in Chapter 2). The reward $r_{t+1}$ is the reward received by the agent after doing an action.

After calculating the temporal difference, the learning agent is ready to adapt its Q-table for each of the fuzzy rules $l \in$ fuzzy rules. The Q-table is updated as follows,

$$
q_{t+1}(l, a)=q_{t}(l, a)+\alpha \Delta_{t+1} \phi_{t}^{l}
$$

Where $\alpha$ is the learning rate.

Exploration and exploitation is done by selecting a random action from action set $A$ with probability $\epsilon$ (exploration rate). We call the forgetting factor $\gamma$, learning rate $\alpha$, and exploration rate $\epsilon$ as the Learning Factors. The FQL algorithms is shown in 
Algorithm 5. This FQL was implemented in [48] and [49].

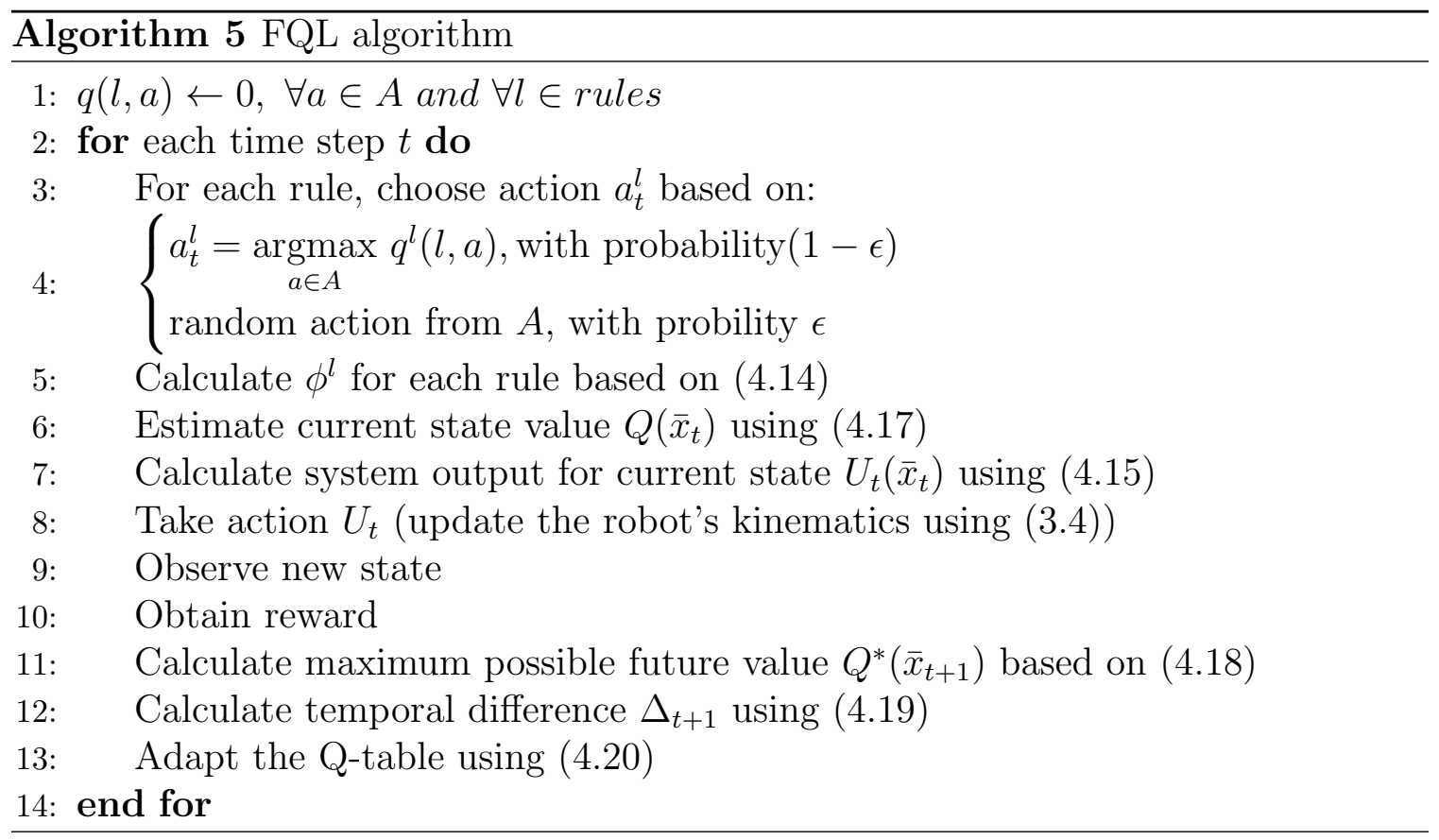

\subsection{Fuzzy Actor Critic Learning (FACL)}

In case the action space is unknown and can not be estimated through the expected action set $A$ (4.16), another possible learning algoirthm can be combined with the fuzzy controller. That is the Fuzzy Actor Critic Learning method [16].

Actor Critic systems (Figure 4.6) consist of 2 parts an actor and a critic. The actor takes the greedy action based on the agent's current state-value. Thus the actor describes the policy structure. Based on the actor actions, the agent will receive a reward from the environment. The critic estimates the states' values based on the policy followed by the actor. Then using the temporal difference error (described in Sec. 2.3), the critic will adapt. Consequently, the actor will adapt as well.

We use the same fuzzy controller described in Sec. 4.1 and Sec. 4.2. The output 


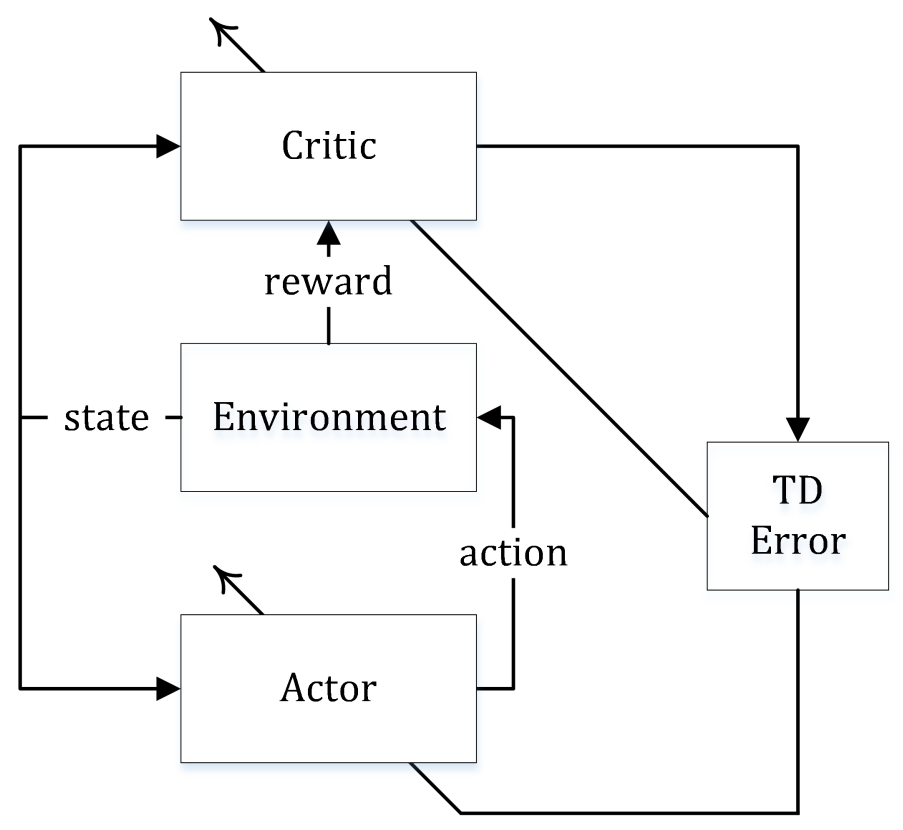

Figure 4.6: Actor Critic Logic System Diagram

of the fuzzy controller is the same as 4.15). A random noise $v(0, \sigma)$ is added to the controller's output to produce exploration. The noisy output becomes $U_{t}^{\prime}$.

The actor's output $a^{l}$ is no longer a constant. It will adapt based on the TD error. One can adapt $a^{l}$ as [16],

$$
a_{t+1}^{l}=a_{t}^{l}+\beta \operatorname{sign}\left\{\Delta \frac{U_{t}^{\prime}-U_{t}}{\sigma}\right\} \phi_{t}^{l}
$$

Where $\Delta$ is the temporal difference error. Only the sign of the temporal difference is used to avoid large changes in the wrong direction. The variable $\beta$ is the actor's learning rate and it controls the step size of the adaptation for the actor output.

The output of the critic (the estimated value) can be calculated as,

$$
\hat{V}_{t}=\sum_{l=1}^{N} \phi^{l} \zeta
$$

Where $\zeta$ is the center of the critic fuzzy rules. 
Once the estimated value is calculated, the temporal difference difference 2.13) can be rewritten as:

$$
\Delta=r_{t+1}+\gamma \hat{V}_{t+1}-\hat{V}_{t}
$$

One then updates $\zeta$ as,

$$
\zeta_{t+1}^{l}=\zeta_{t}^{l}+\alpha \Delta \phi_{t}^{l}
$$

Such that $\alpha$ is the critic's learning rate.

Algorithm 6 describes the Fuzzy Actor Critic Learning algorithm:

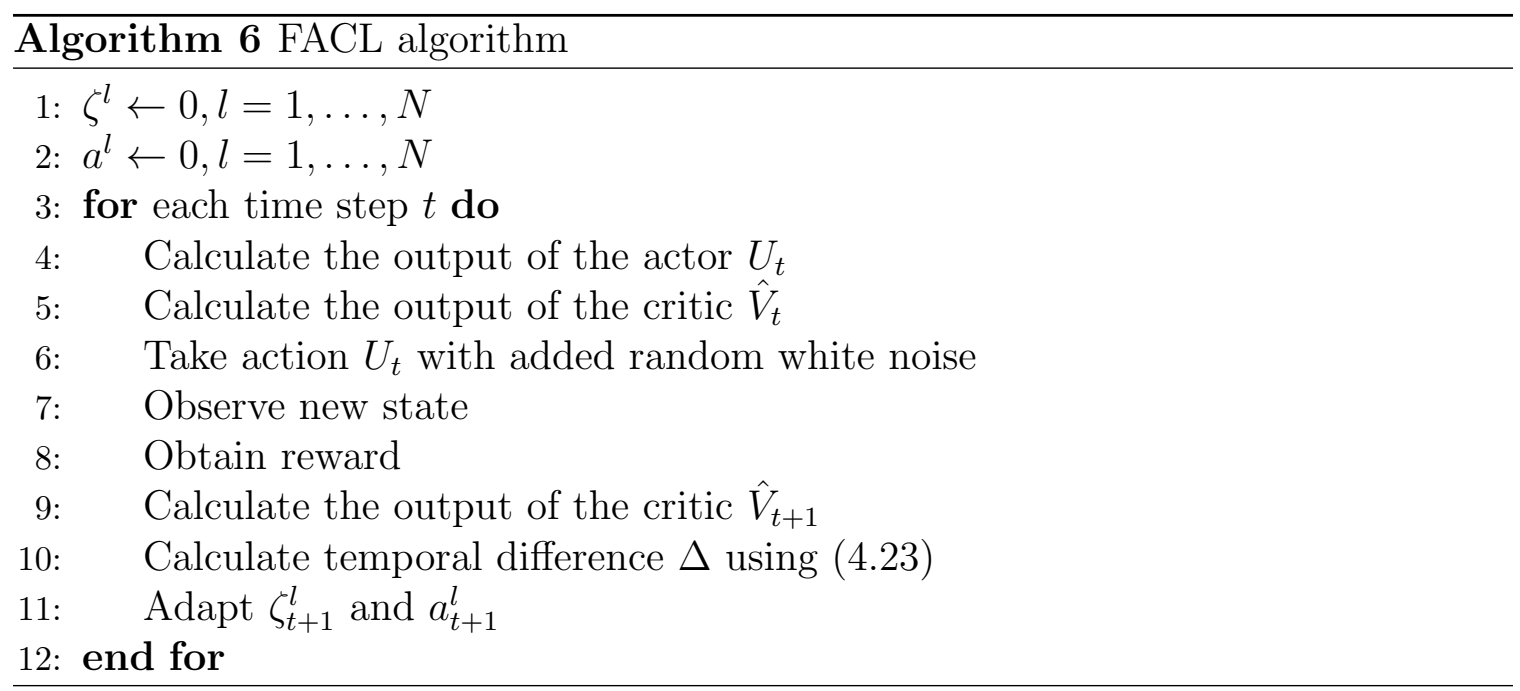

\subsection{Reward Function Selection}

Reinforcement Learning can have two different types of rewards. They are terminal rewards or instantaneous rewards. In our game, a terminal reward will be given only if the invader reaches the territory. However, this will make it difficult for the invader to learn when it is impossible to reach the territory. Also, it will cause slow learning. That is because the invader will have to take many actions before it can reach the territory. Thus, it will not know if it is doing the correct action for a long time. On 
the other hand, an instantaneous reward will always inform the agent how well the agent is doing at each time-step.

We propose using an instantaneous reward function. It is difficult to select the reward function. To construct our reward function, we divide our game into two games.

The first game is a single invader with no guards. The invader's goal is to reach the territory. In that case we can describe our instantaneous reward by how much the agent got closer to the territory. That is:

$$
r_{t+1}^{p 1}=\operatorname{dist}_{I T}(t)-\operatorname{dist}_{I T}(t+1)=\delta_{I T}
$$

Where $\operatorname{dist}_{I T}(t)$ is the euclidean distance between the invader and the territory at time $t$. Thus, this constructs the instantaneous reward for the first part of the game.

We describe the second game as a pursuer evader game. Such that the invader is trying to run away from the guard and no territory is involved. In other words, the invader is trying to increase the distance between itself and the guard. Hence, we can describe the reward in this part of the game as how far the agent went from the guard. That is:

$$
r_{t+1}^{p 2}=\operatorname{dist}_{I G}(t+1)-\operatorname{dist}_{I G}(t)=-\delta_{I G}
$$

Such that $\operatorname{dist}_{I G}(t)$ is the euclidean distance between the guard and the invader at time $t$.

Once we have constructed these two rewards, we propose combining both rewards with ratios $K$ and $J$ to produce the agent's full instantaneous reward.

$$
r_{t+1}=K \delta_{I T}-J \delta_{I G}
$$


We describe $K$ as a constant that describes the importance of getting closer to the territory. The larger $K$ is, the faster the agent will go to the territory. Similarly, $J$ describes the importance of travelling furthest from the guard. Since the guard is always faster than the invader, dist ${ }_{I G}$ will never increase. However, the invader's actions will chose how slowly the distance (between the guard and the invader) decreases. Thus, $J$ describes the importance of controlling the distance decrease rate between the guard and the invader.

\subsection{Summary}

We used the basis for fuzzy "IF... THEN" rules to build our fuzzy system. Our control system consisted of Takagi and Sugeno fuzzy rules with constant consequent, a singleton fuzzifier, product inference engine and average weighted defuzzifier. The fuzzy system is used to provide an estimate for the game's state. The center of the fuzzy controller is a constant that belongs to an action set that describes the possible headings (that is for the FQL use) or an adaptive value for the actor (that is for the FACL use). Both the FQL and the FACL algorithms combine the fuzzy system with reinforcement learning. The output of the system was chosen to select a steering heading.

We proposed selecting our controller inputs as: the angle between the invader's line of sight with the territory and the global $\mathrm{x}$-axis, and the Manhattan distance between the guard and the invader. This resulted in a total of 3 inputs and 225 possible combinations of rules.

Furthermore, we proposed an instantaneous reward that aims at decreasing the distance between the invader and the territory, and that will control the rate of decrease in distance between the guard and the invader. 


\section{Chapter 5}

\section{Simulation and Experimental Results}

In this chapter, we will simulate the problem described in Chapter 3 and use the control system described in Chapter 4. We will demonstrate our simulations for the grid and differential games. The chapter concludes with laboratory experimental results conducted at the Royal Military College of Canada in Kingston, Ontario. These experiments will demonstrate how the agent performed in a real-life environment.

\subsection{Grid World Simulation}

In this section, we will use different learning algorithms described in Chapter 2 to solve the $2 \times 2$ grid world example provided in Sec. 3.1. The simulations in this section is a replication of the results demonstrated in [4]. We have done this simulation as one small step towards solving our larger problem. We will investigate the results when both players are learning, as well as, when only one player is learning.

\section{Both Players Learning}

In our simulations, we used reinforcement learning on both players to let them obtain the optimal policy. As explained in Sec. 3.1, the output of the first state will 


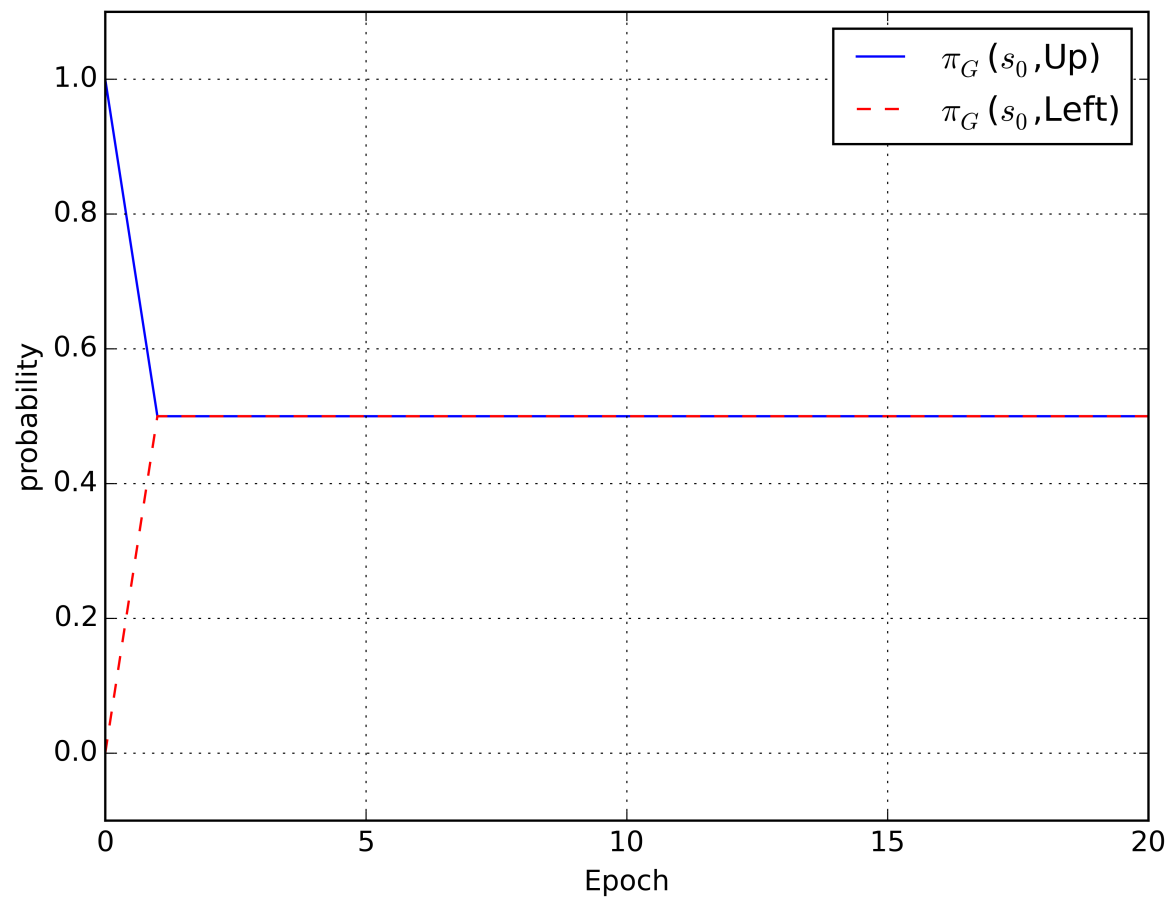

Figure 5.1: Grid World Q-Minimax Simulation Result - Guard's Strategy

immediately inform us if the guard can capture the invader or not.

We use the Q-Minimax learning algorithm as our learning algorithm for both players. We run the simulation multiple times, and every time we record the players' new policy. Each simulation starts with the players in the initial state $s_{0}$. We set our learning rate $\alpha$ to decay for both players after each simulation epoch as follows:

$$
\alpha_{j}=\frac{1}{10+j / 2}
$$

Where $j$ is the simulation epoch number.

The simulation results are presented in Figure 5.1 and Figure 5.2. Figure 5.1 demonstrates the guard's policy $\pi_{G}$ at $s_{0}$. Figure 5.2 demonstrates the invader's policy $\pi_{G}$ at $s_{0}$. It can be concluded from the simulations that the agents using the Q- 


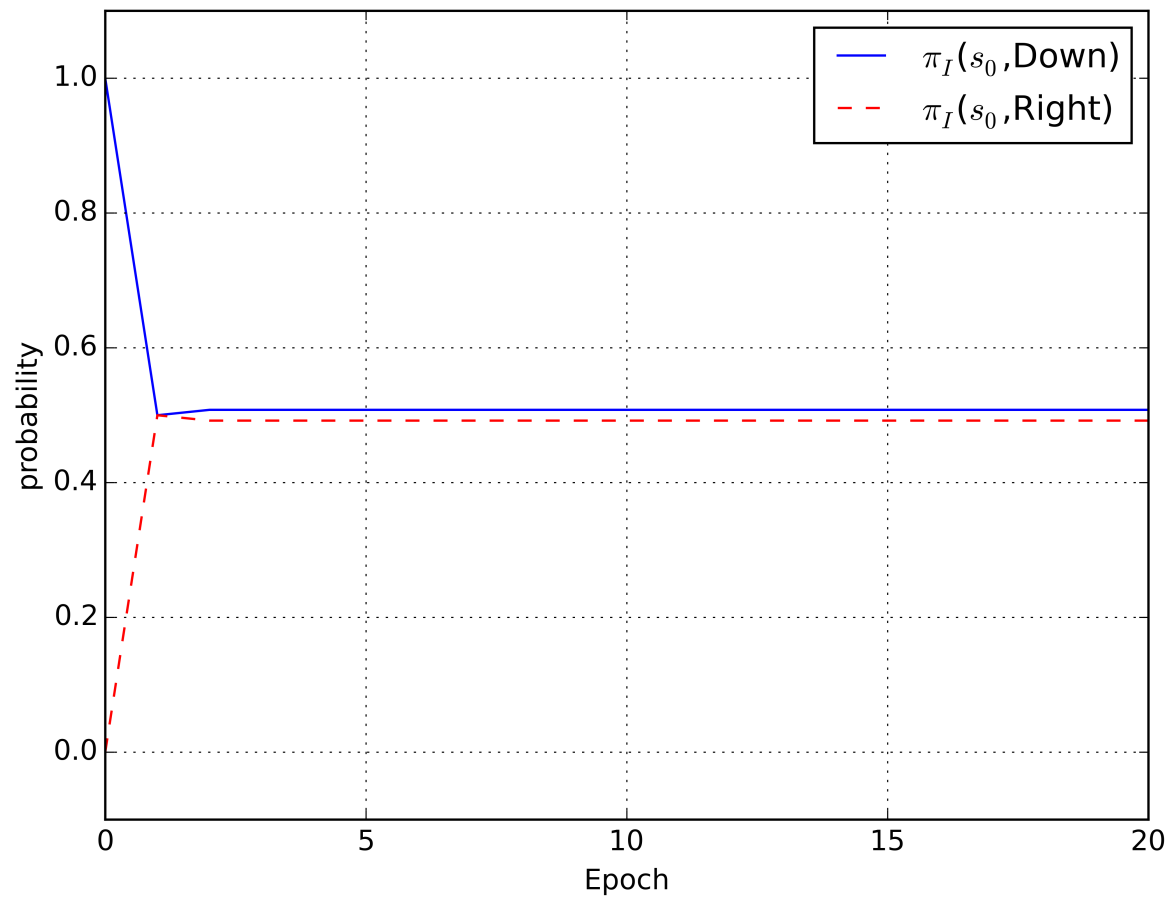

Figure 5.2: Grid World Q-Minimax Simulation Result - Invader's Strategy

Minimax algorithm converged to policies as given by the theoretical value. Such that, the probability of taking any action for any player in the initial state is $50 \%$. This demonstrates the Nash Equilibrium policy for both player $\left(\pi_{G}^{*}\left(s_{0}\right), \pi_{I}^{*}\left(s_{0}\right)\right)$, which is the best they can do under the current game conditions.

In our second simulation, we used the WoLF-PHC algorithm. The same simulation configuration was done. Again, we recorded the players' policy at $s_{0}$ in each simulation. However, we choose our learning rates as:

$$
\begin{array}{r}
\alpha_{j}=\frac{1}{10+j / 10000} \\
\delta_{w_{j}}=\frac{1}{10+j / 2} \\
\delta_{l_{j}}=\frac{3}{10+j / 2}
\end{array}
$$

We chose the learning rates to have a small initial values so the agent's strategy will 


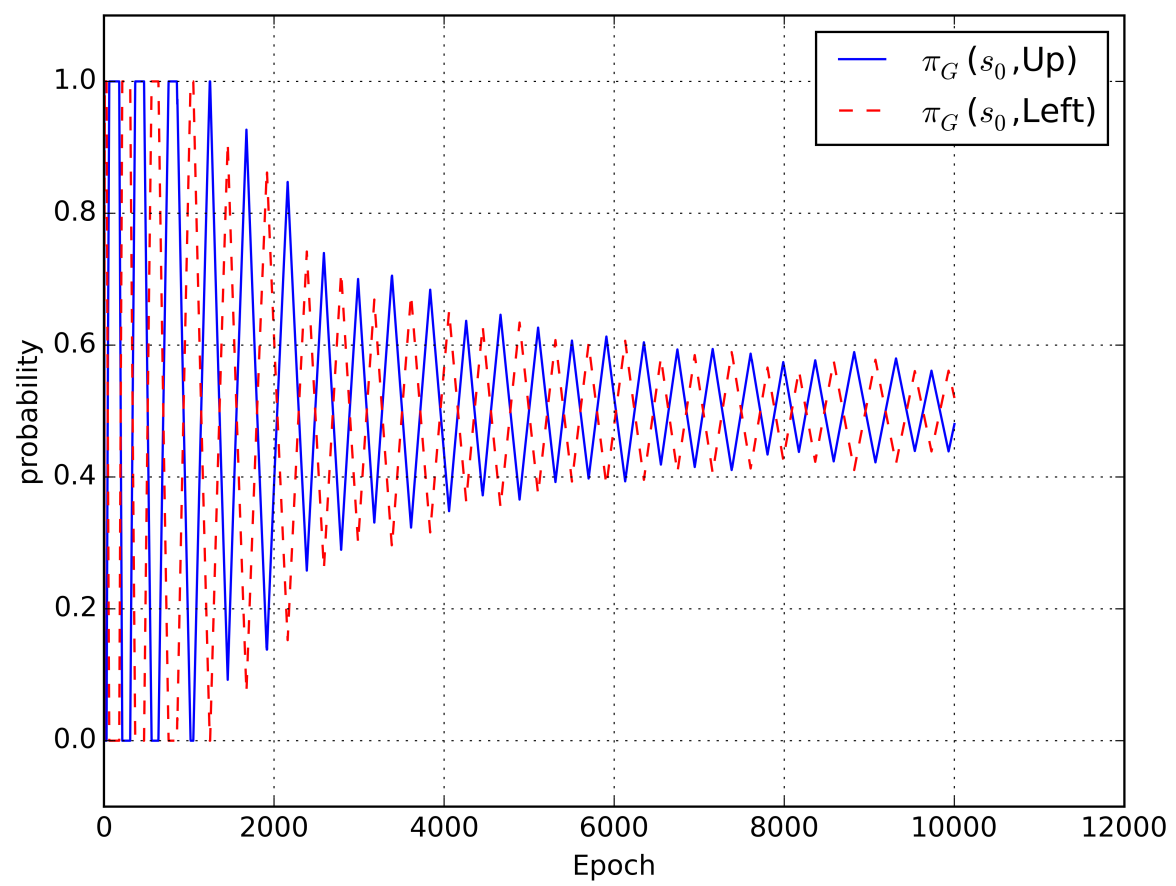

Figure 5.3: Grid World WoLF-PHC Simulation Result - Guard's Strategy

not oscillate. We chose a slow decay since the agent is expected to converge their strategy in a longer time than Q-Learning.

Figure 5.3 shows the results for the guard's policy and Figure 5.4 shows the results for the invader's policy. It can be observed that the agents also converged to the same expected policy $\left(\pi_{G}^{*}\left(s_{0}\right), \pi_{I}^{*}\left(s_{0}\right)\right)$. However, the convergence was slower than seen in Q-Minimax algorithm. The Q-Minimax algorithm converged faster than WoLF-PHC algorithm because Q-Minimax computes the optimal solution every time step using linear programming. The WoLF-PHC does not compute the optimal solution every time step. Thus, the convergence comes at the computational cost. 


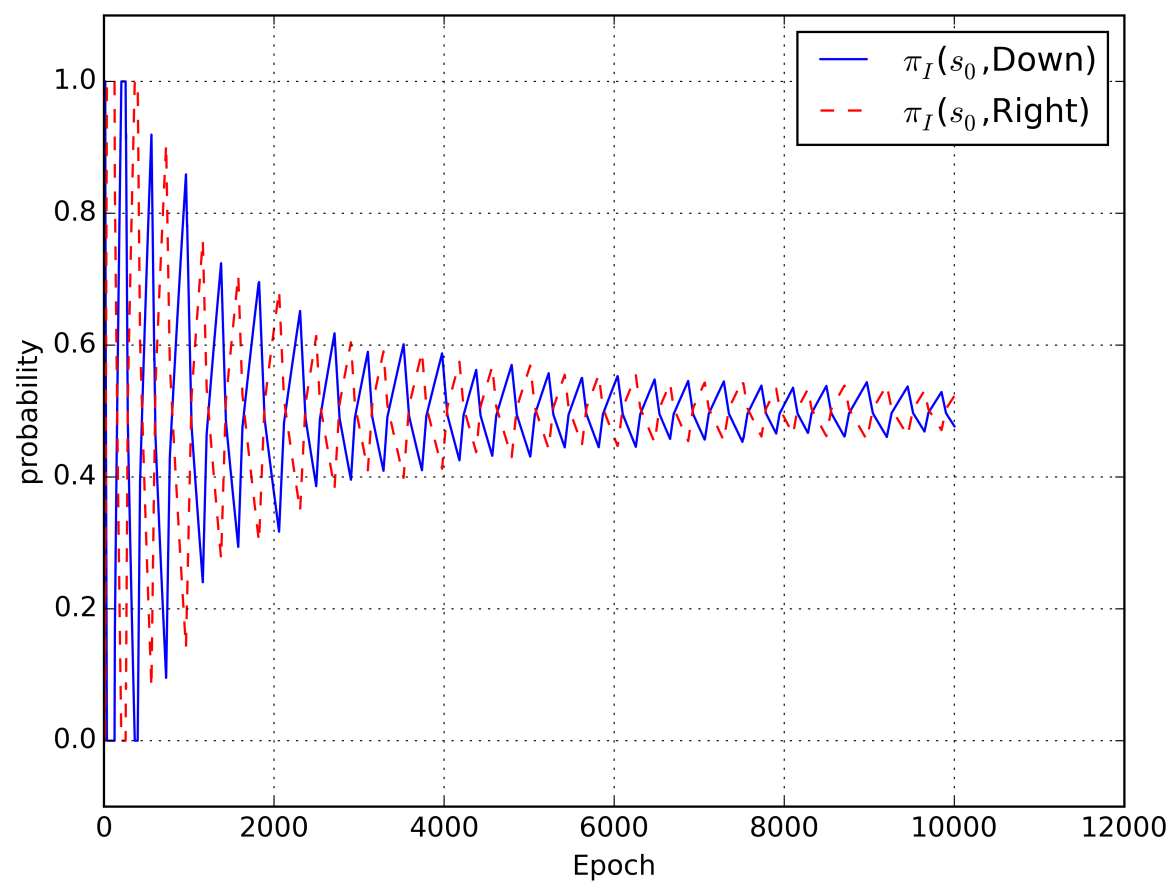

Figure 5.4: Grid World WoLF-PHC Simulation Result - Invader's Strategy

\section{Only Invader Learning}

In the following simulations, the guard will follow a pure strategy, and we will let the invader adapt to it. In other words, the guard will not learn or adapt its strategy.

In our simulations we fix the guard's strategy to always select the action left. A rational invader should then avoid square 3 in Figure 3.1 and move right. Figure 5.5 demonstrates the simulation results, for Q-Minimax algorithm, by showing the invader's strategy at $s_{0}$. Similarly, we run the same simulations using the WoLF-PHC. The results for the WoLF-PHC algorithm can be seen in Figure 5.6.

Figure 5.5 and Figure 5.6 demonstrate that the agent, using either of the algorithms, adapted its strategy to avoid square 3 and pick action right at probability of 100\%. Thus, it will be able to maximize its reward and avoid being captured at all times. 


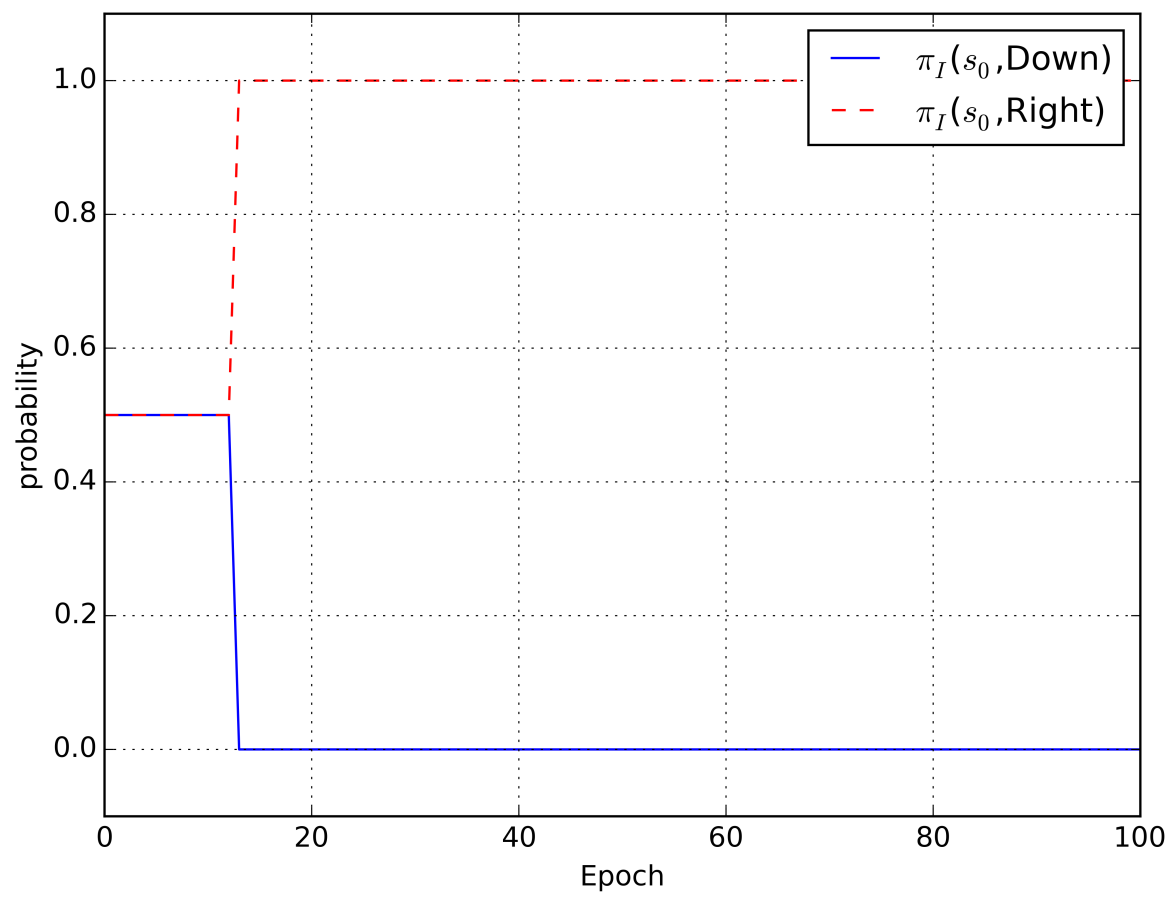

Figure 5.5: Grid World Q-Minimax Simulation Result - Invader's Strategy vs. Guard's pure strategy

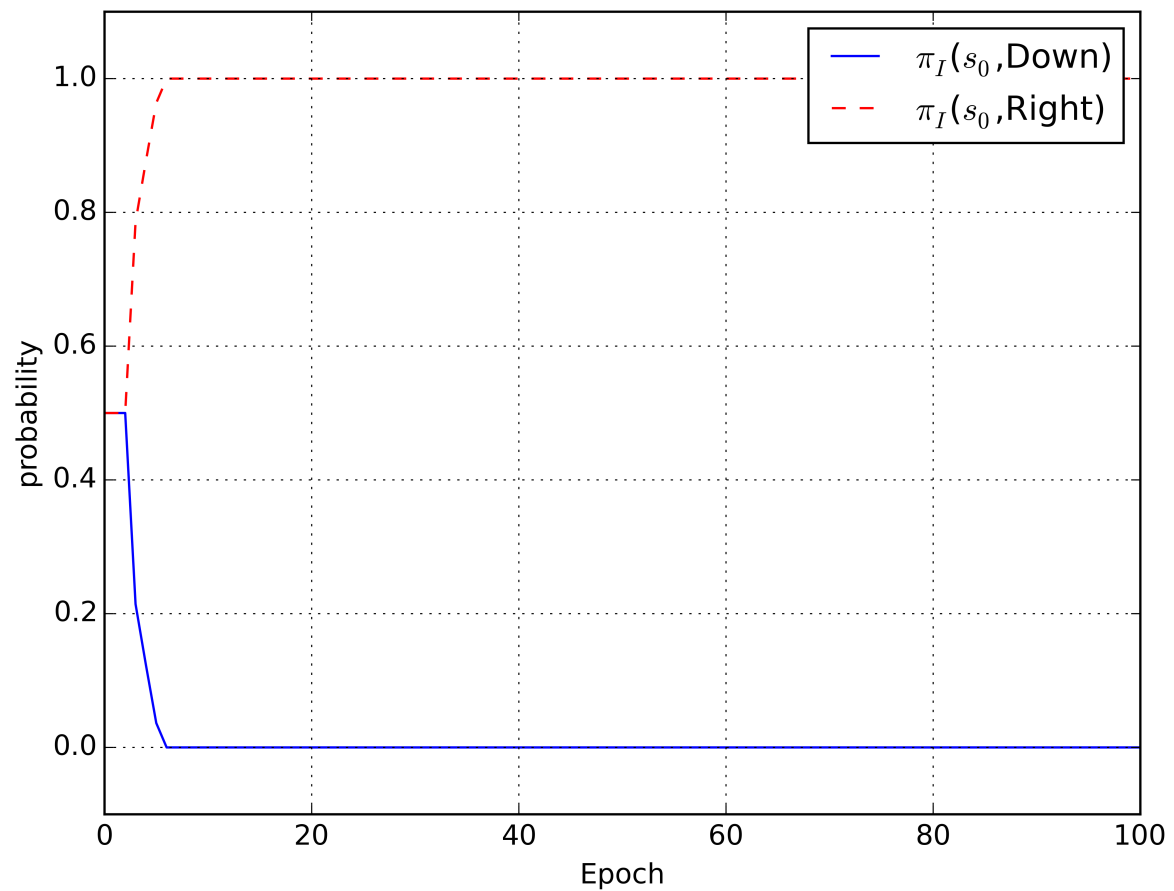

Figure 5.6: Grid World WoLF-PHC Simulation Result - Invader's Strategy vs. Guard's pure strategy 


\subsection{Single-Player Game (Invader Reaching Terri- tory)}

To start our simulations for the differential version of the game, we considered implementing the simple version of the game described in Sec. 4.5. Such that, the game only has one player, which is the invader. This game was investigated by the author in $[5]$.

We decided to create two different sets of simulations to solve this version of the game. First, we will replicate the control system used in [5]; however, we will be using our non-holonomic constraints. Second, we will use the input $\theta$ described in Sec. 4.2 .

This was done to:

1. Verify our simulation model design on a simpler game version.

2. To easily investigate any errors.

3. To compare the performance between two different controllers.

4. To compare between the FQL and the FACL algorithms.

In all our differential game simulations, the sampling frequency was chosen as $T=10 \mathrm{mSec}$. Equation (3.4) was solved in the simulations using Euler integration.

\subsubsection{Replica Controller}

The author in [5] used the agent's coordinates $\left(x_{I}, y_{I}\right)$ as the control inputs for this version of the game. The fuzzy sets, for each of the inputs $\left(x_{i} \in\left[x_{I}, y_{I}\right]\right)$, were chosen as:

- ZE: $x_{i}$ is near 0 (Zero) 


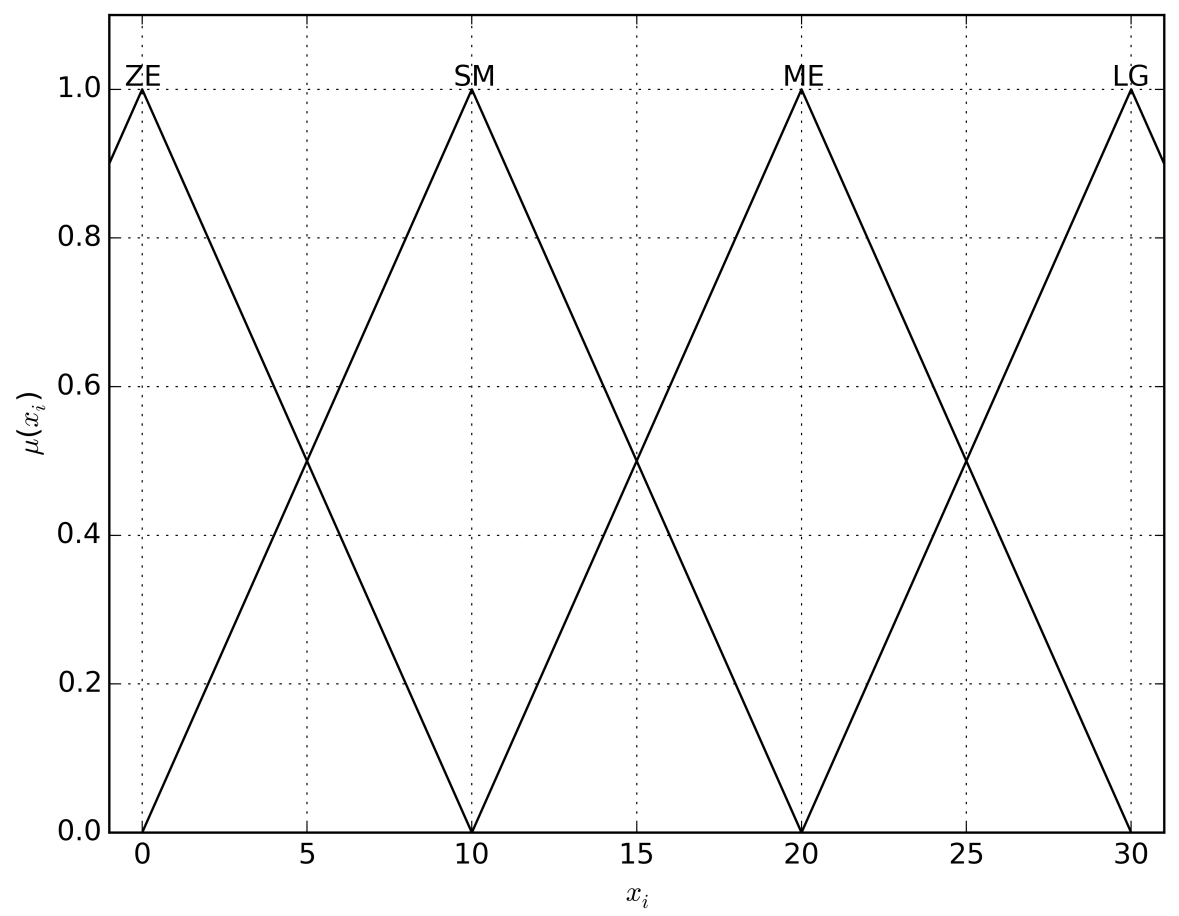

Figure 5.7: Membership function using coordinates signals as inputs

- SM: $x_{i}$ is near 10 (Small)

- ME: $x_{i}$ is near 20 (Medium)

- LG: $x_{i}$ is near 30 (Large)

The membership functions for the inputs were chosen as triangular functions as shown in Figure 5.7 .

After setting the invader's speed to 1 unit/second $\left(v_{I}=1\right.$ unit/sec $)$ and the angular speed can be either $-0.5,0$, or $0.5 \mathrm{rad} / \mathrm{sec}\left(\left|\omega_{I_{\max }}\right|=0.5 \mathrm{rad} / \mathrm{sec}\right)$, we run the singleplayer game version simulation using the FQL and the FACL algorithms described in Chapter 4. Then we compare both algorithms based on the agent's performance. Both the FQL and the FACL algorithms were run 400 epochs. An epoch is a game that starts at the players' initial position and ends at termination time. The agent 
uses the learnt parameters (Q-table) from the previous epochs at the start of the new epoch.

In the following simulations, the territory is a circle placed at coordinates $\left(x_{T}, y_{T}\right)=(20,15)$ with a radius of 2units. The invader's initial position is set as $\left(\left(x_{I}, y_{I}\right)=(5,5)\right)$. Termination time was set to 50 seconds. This means if the invader was unable to reach the target in 50 seconds, the game will terminate (there are no guards in this game). Otherwise, the game terminates as soon as the invader reaches the territory.

\section{FQL Simulations}

In our FQL simulations, we set the learning rates as follows:

$$
\begin{gathered}
\alpha=0.99^{j} \alpha_{0} \\
\gamma=0.99^{j} \gamma_{0} \\
\epsilon=0.99^{j} \epsilon_{0}
\end{gathered}
$$

Such that, $\alpha_{0}=0.1, \gamma_{0}=0.9$, and $\epsilon_{0}=0.3$. Where $j$ represents the epoch's number.

Figure 5.8 shows the invader's trajectory for the first simulation epoch. As seen, the invader was not able to reach the territory. Figure 5.9 shows the invader trajectory after learning, that is simulation epoch 400. It can be observed that the invader was able to reach the territory.

To see how the agent progressed, we recorded the agent's payoff after each epoch (400 epochs), then we repeated the same simulation 10 times. The agent's payoff is our own evaluation of how well the agent did as described in Chapter 3. Figure 5.10 displays the average payoff of the 10 simulations after each epoch. It can be observed that the agent converged to reaching the territory after approximately 150 epochs. 


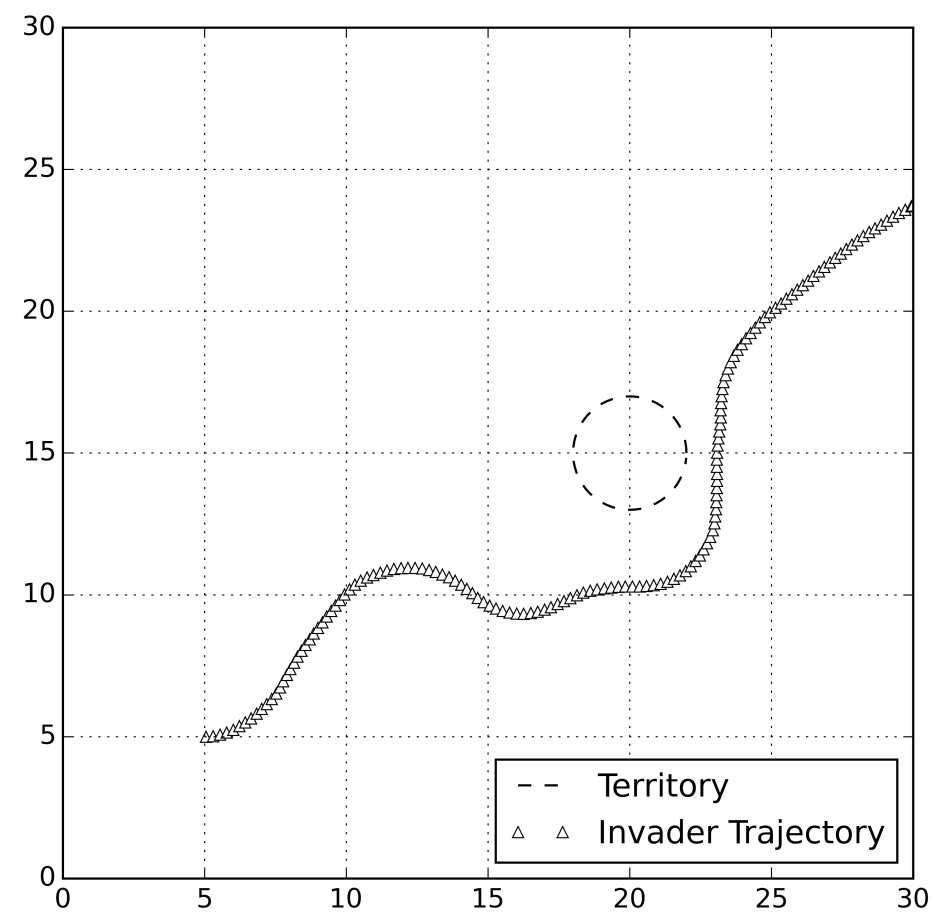

Figure 5.8: FQL - Invader Reaching Territory - Epoch 001

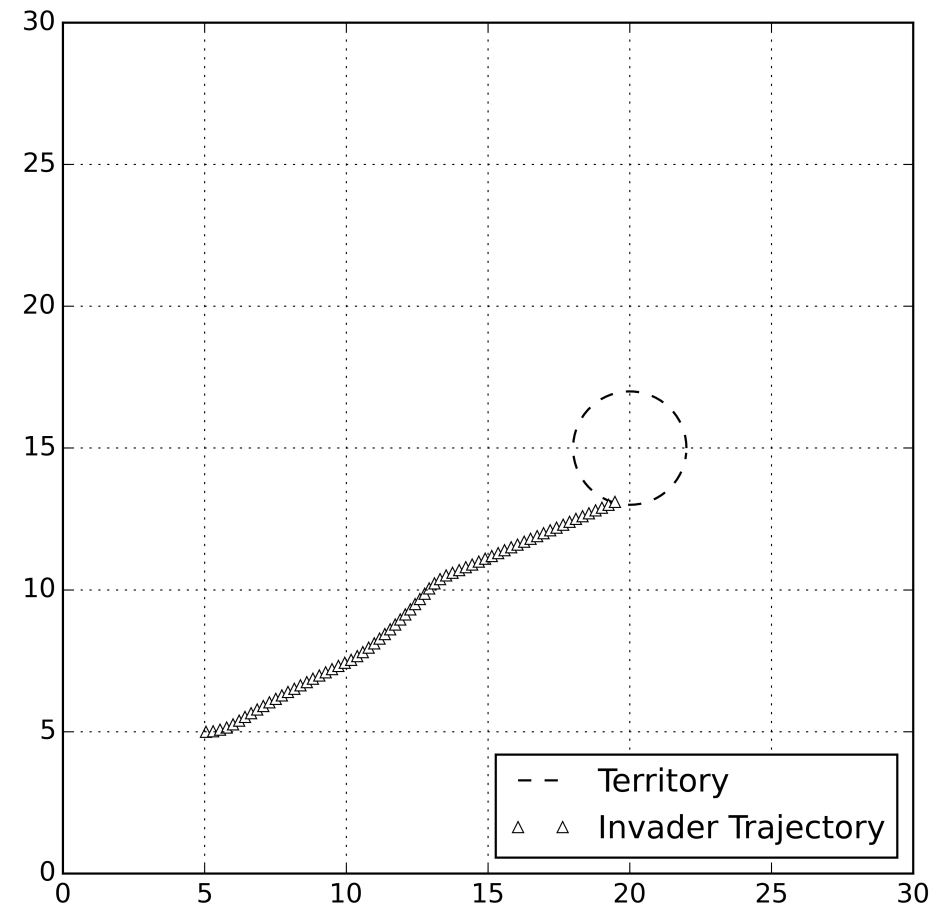

Figure 5.9: FQL - Invader Reaching Territory - Epoch 400 


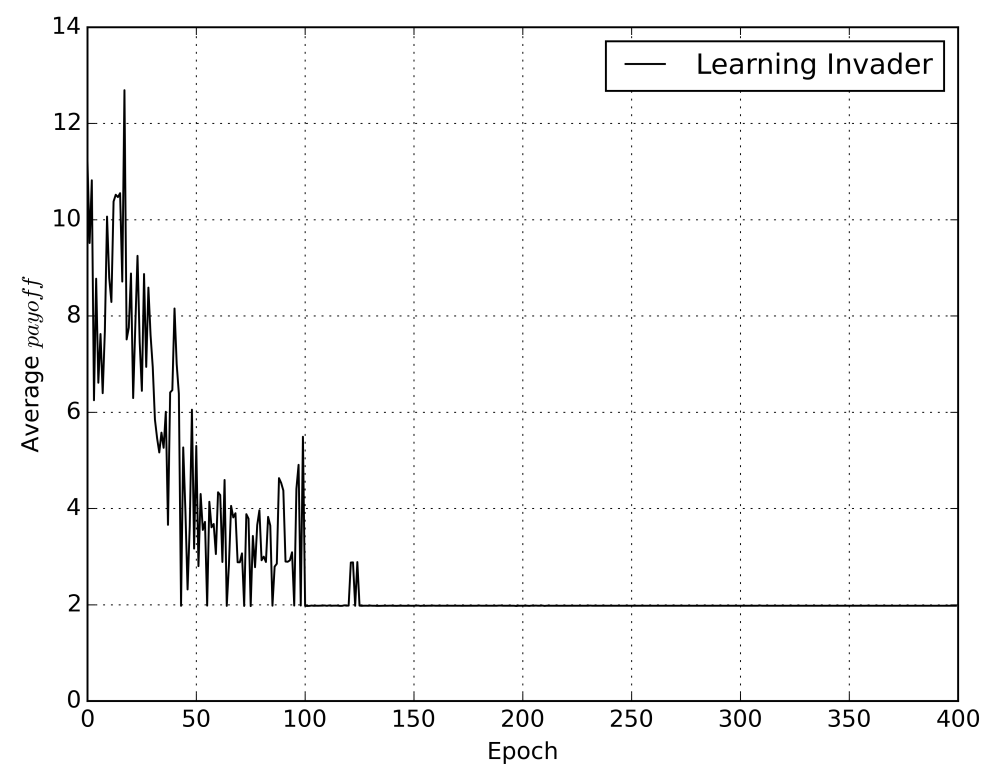

Figure 5.10: FQL - Invader Reaching Territory - Payoff

\section{FACL Simulations}

Similarly, we also ran the same simulation using the FACL. However, the learning rates were set as follows:

$$
\begin{aligned}
& \alpha=\frac{1}{0.01 \times j+10} \\
& \beta=\frac{1}{0.01 \times j+20} \\
& \gamma=0.9
\end{aligned}
$$

Figure 5.11 shows how the agent using the FACL algorithm performs in its first epoch. The agent was unable to reach the territory at first. Figure 5.12 shows the last epoch, such that the invader was able to reach the territory. It can also be observed that the agent did not use the shortest path and it took some turns before it reached the territory. 
We repeated the simulation 10 times to investigate the agent's performance. Figure 5.13 displays the agent's average payoff for the 10 simulations. In our simulations, the agent using FACL reached the territory by the 300th epoch.

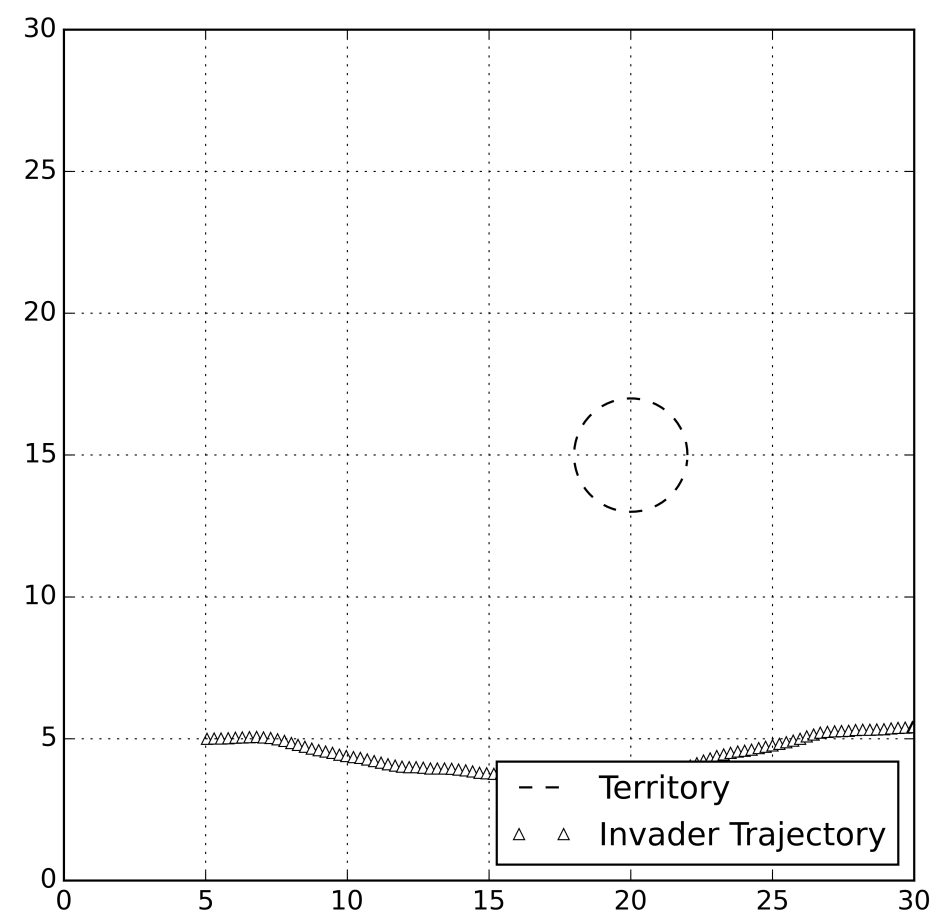

Figure 5.11: FACL - Invader Reaching Territory - Epoch 001 


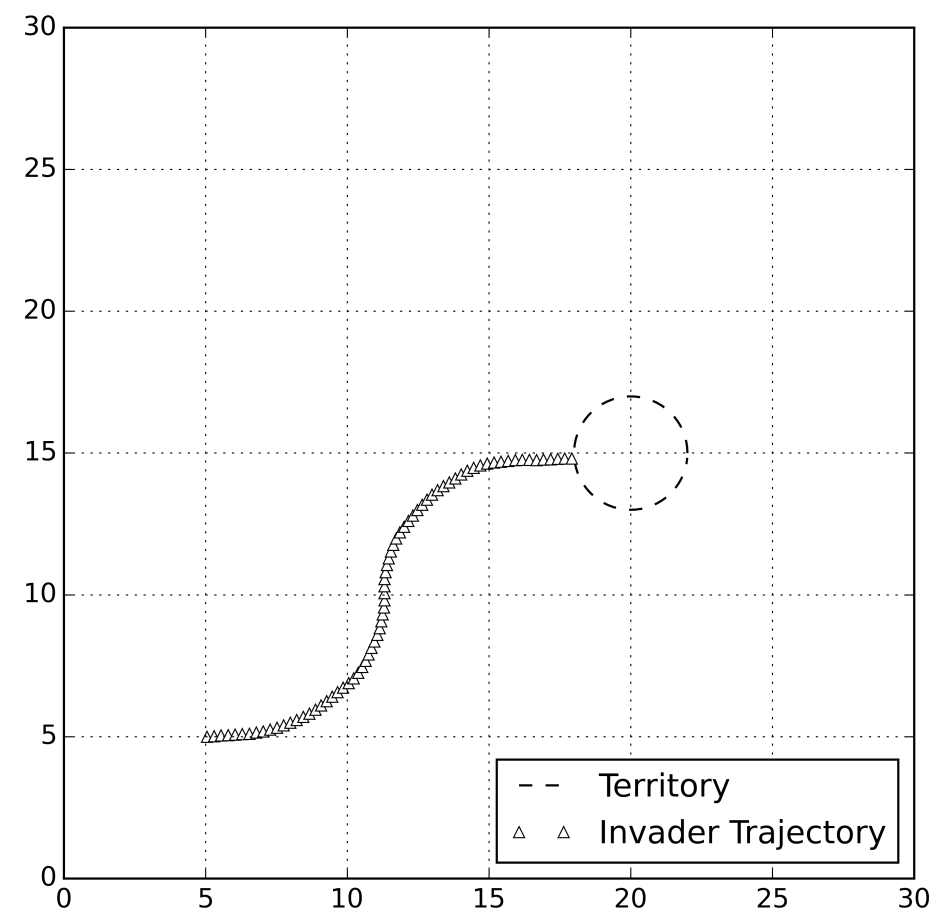

Figure 5.12: FACL - Invader Reaching Territory - Epoch 400

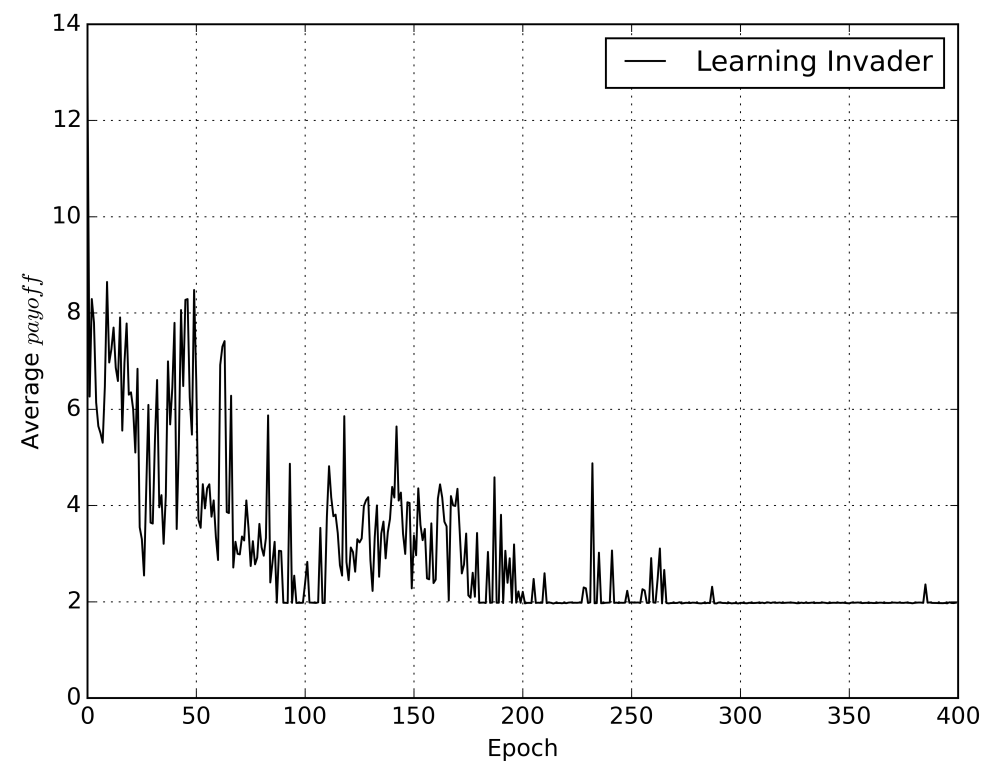

Figure 5.13: FACL - Invader Reaching Territory - Payoff

To further compare between the two algorithms, we look into the average game termination time in both algorithms (Figure 5.14). Results from this figure will be 
discussed in next subsection.

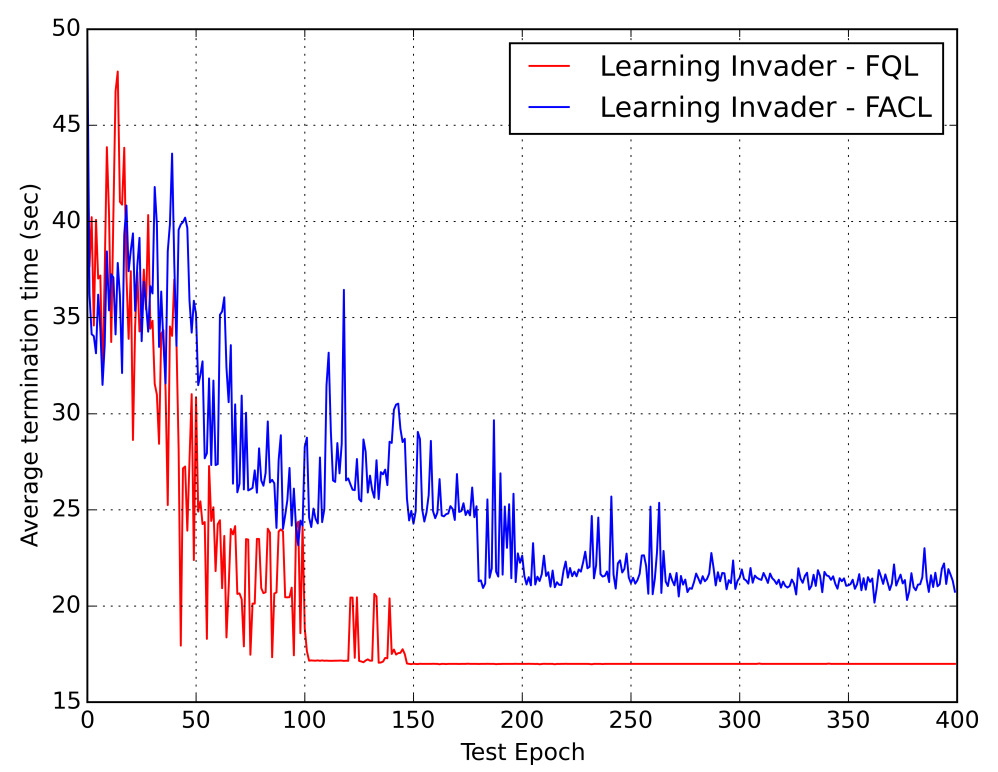

Figure 5.14: Termination Time average - FQL vs. FACL

\subsubsection{Single Input Controller}

We now repeat the simulations using the same characteristics as in Sec.5.2.1; however, we only use a single input $\theta$ (described in Sec. 4.2).

\section{FQL Simulations}

We run the FQL algorithm using the same learning rates as in (5.6) to (5.7). Figure 5.15 shows how the invader performed in its first test epoch. Figure 5.16 shows how the invader performed in its last test epoch after learning. It can be observed that the agent went in a straight towards the territory after learning. 


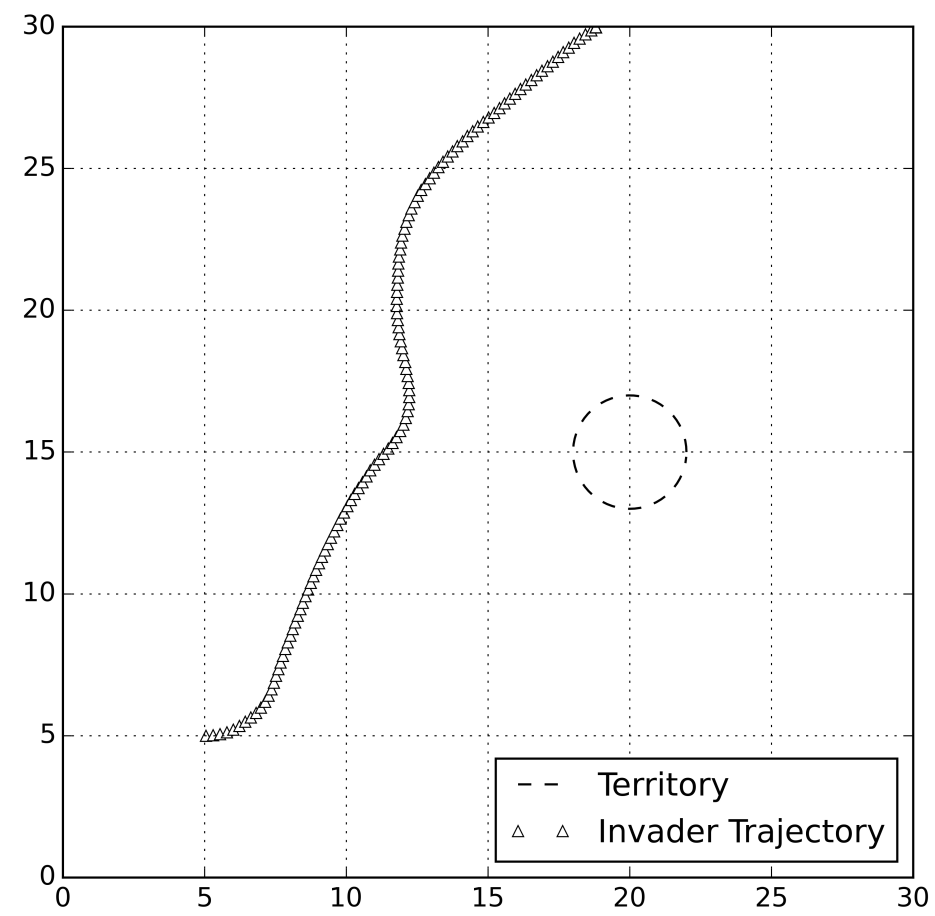

Figure 5.15: FQL - Invader Reaching Territory - Single Input Controller - Epoch 001 


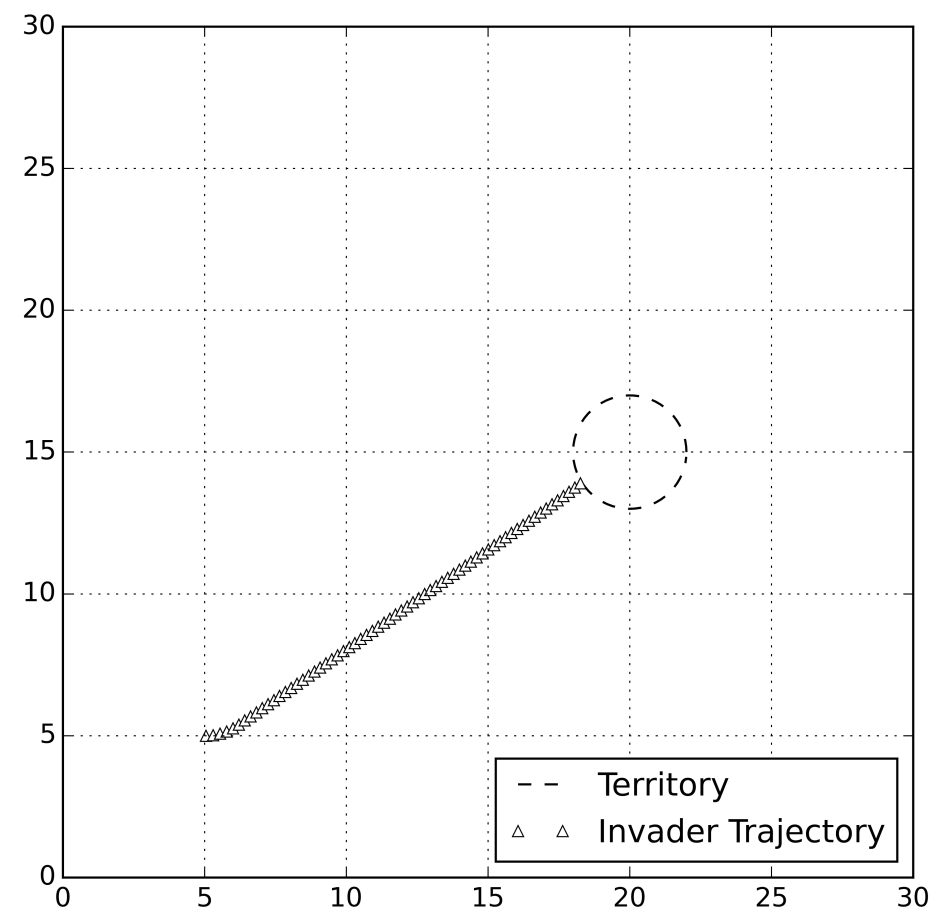

Figure 5.16: FQL - Invader Reaching Territory - Single Input Controller - Epoch 400

Figure 5.17 demonstrates the invader's average payoff for 10 simulations. It can be observed that the invader was able to converge to reach the territory by the $100^{t h}$ epoch. 


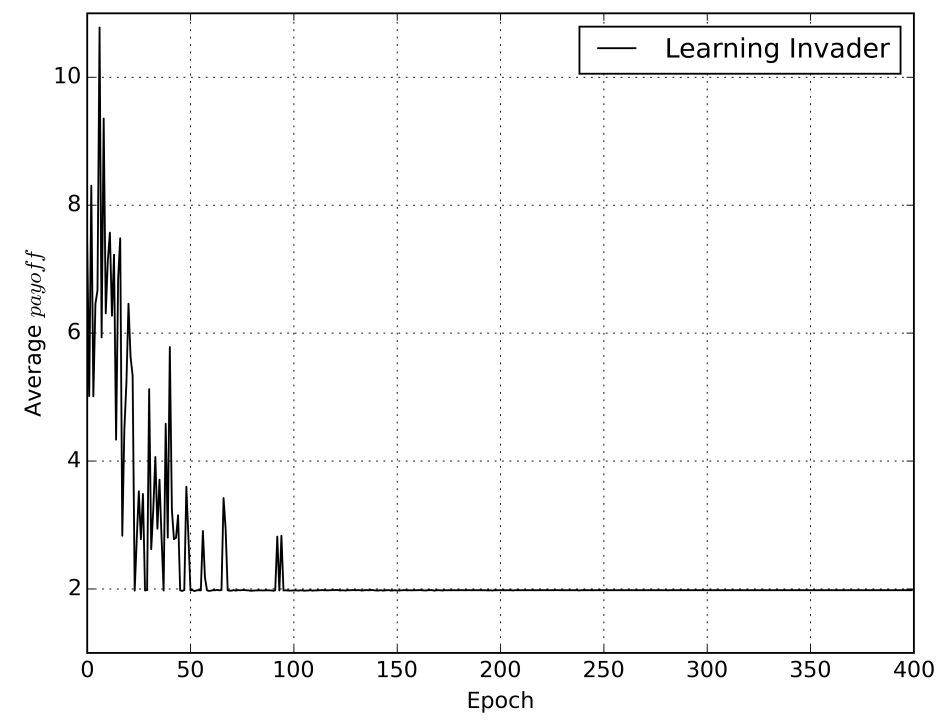

Figure 5.17: FQL - Invader Reaching Territory - Single Input Controller - payoff

\section{FACL Simulations}

We run the FACL algorithm using the same learning rates as in (5.9) to (5.10). Figure 5.18 shows how the invader performed in its first test epoch. Figure 5.19 shows how the invader performed in its last test epoch after learning. It can be observed that the agent did not use the shortest path. 


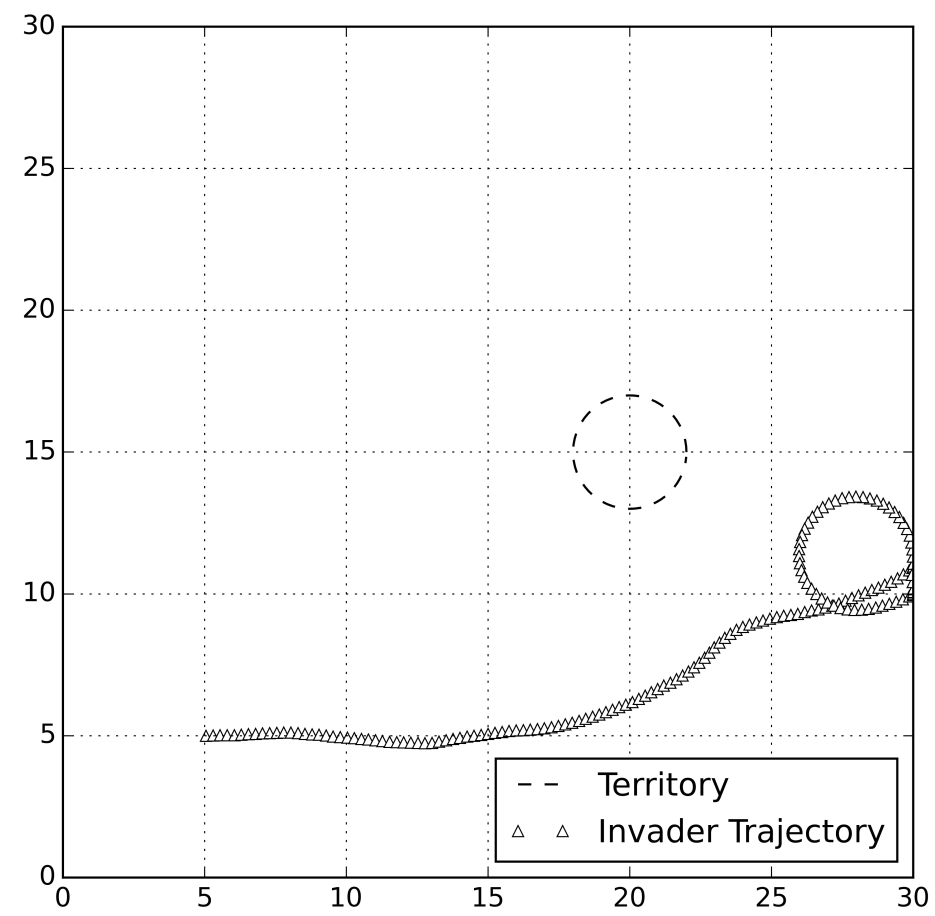

Figure 5.18: FACL - Invader Reaching Territory - Single Input Controller - Epoch 001 


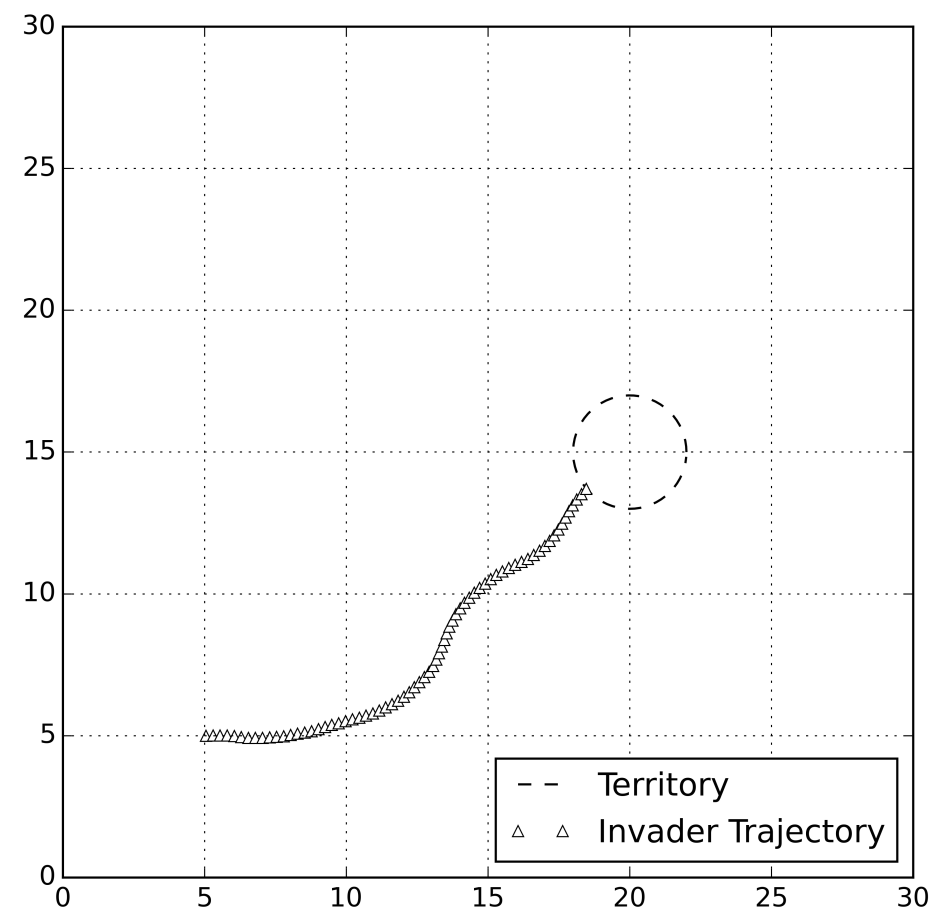

Figure 5.19: FACL - Invader Reaching Territory - Single Input Controller - Epoch 400

Figure 5.20 demonstrates the invader's average payoff for 10 simulations. The agent was able to converge and reached the territory by the $150^{\text {th }}$ epoch.

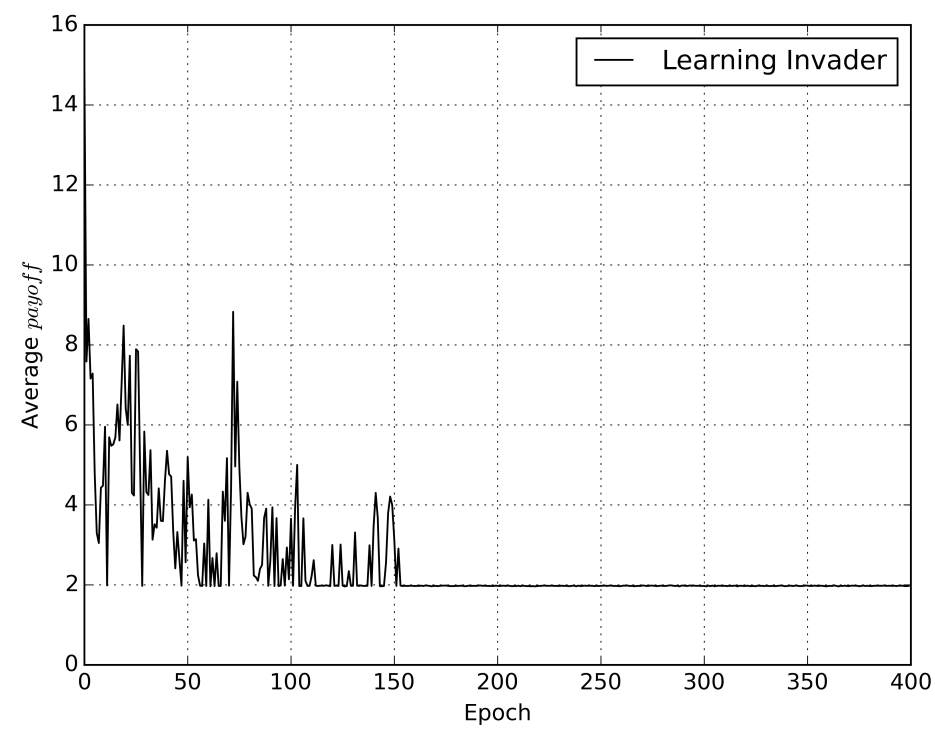

Figure 5.20: FACL - Invader Reaching Territory - Single Input Controller - payoff 
We again compare between both the FQL and the FACL algorithms by checking how fast was the invader able to reach the territory. Figure 5.21 compares the average game termination time for both algorithms.

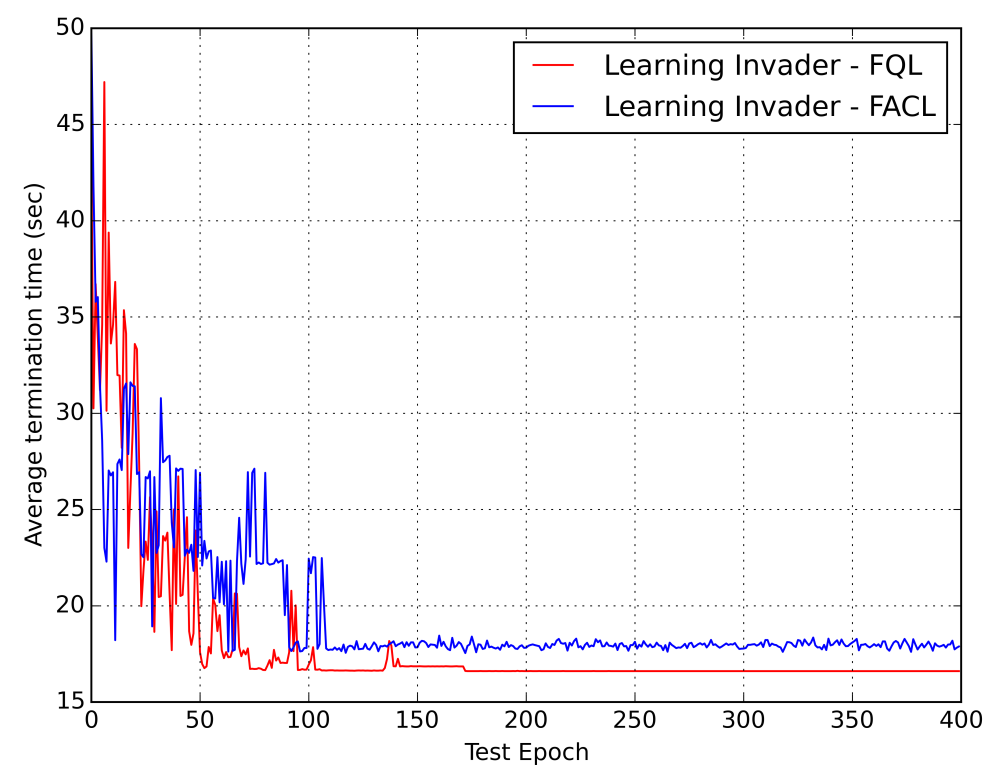

Figure 5.21: Termination Time average - FQL vs. FACL - Single Input Controller

We can observe that in Figure 5.21 the FACL performed better using a single input rather than in Figure 5.14. This is probably due to the simplicity of the controller. Also by comparing both graphs, we can see that using a single controller input, the FQL was able to achieve a smaller termination time. It was reduced from $16.99 \mathrm{sec}$ to $16.60 \mathrm{sec}$.

Using either of the controllers, it can be observed that our agent's model performed better using the FQL algorithm. It converged to reaching the territory using the FQL algorithm faster than using FACL algorithm. Also, it was able to terminate the game (reach the territory) in a shorter time compared to FACL. We can also observe that our single input allows the agent to perform better using either of the algorithms. Thus, we conclude that using FQL algorithm is a better choice to be used with our system. 
After completing the simulations for the simpler game version with a single player, we were able to proceed and add the guard to the simulation as will be seen in the next section.

\subsection{Two-Player Game (Guarding a Territory)}

This section describes the major simulation done to demonstrate how we solved the formulated problem described in Chapter 3 using our customized controller described in Chapter 4 with the FQL algorithm.

\section{Simulation Setup}

We define an epoch as a run of the game until terminal time. An epoch can be a training or a test epoch. In any test epoch, all the learning factors are set to zero. Therefore, there is no learning or exploration taking place. A simulation consists of a set of epochs. Capturing happens when the invader and the guard are 2 units apart $\left(\right.$ dist $\left._{\text {capture }}=2\right)$. Finally, the territory is represented by a circle with a radius of 2 units. The training and testing technique can be realised in Algorithm 7 after the learning factors are initialized. Eq. 3.4 was solved in the simulation using Euler integration.

The territory was placed at coordinates $(20,15)$. The training region was arranged for the guard and the invader to randomly choose a starting position $\left(x_{G 0}, y_{G 0}\right)$ and $\left(x_{I 0}, y_{I 0}\right)$; such that $x_{G 0} \in[0,10], y_{G 0} \in[20,30]$ shown as the dashed area in Figure 5.22 and $x_{I 0} \in[6,10], y_{I 0} \in[6,15]$ shown as the solid area in same figure. The testing starting positions were chosen such that: $P_{I}=(5,5)$ and $P_{G}=(5,25)$ and $\varphi_{I}$ and $\varphi_{G}$ are initialized to zero radians. 

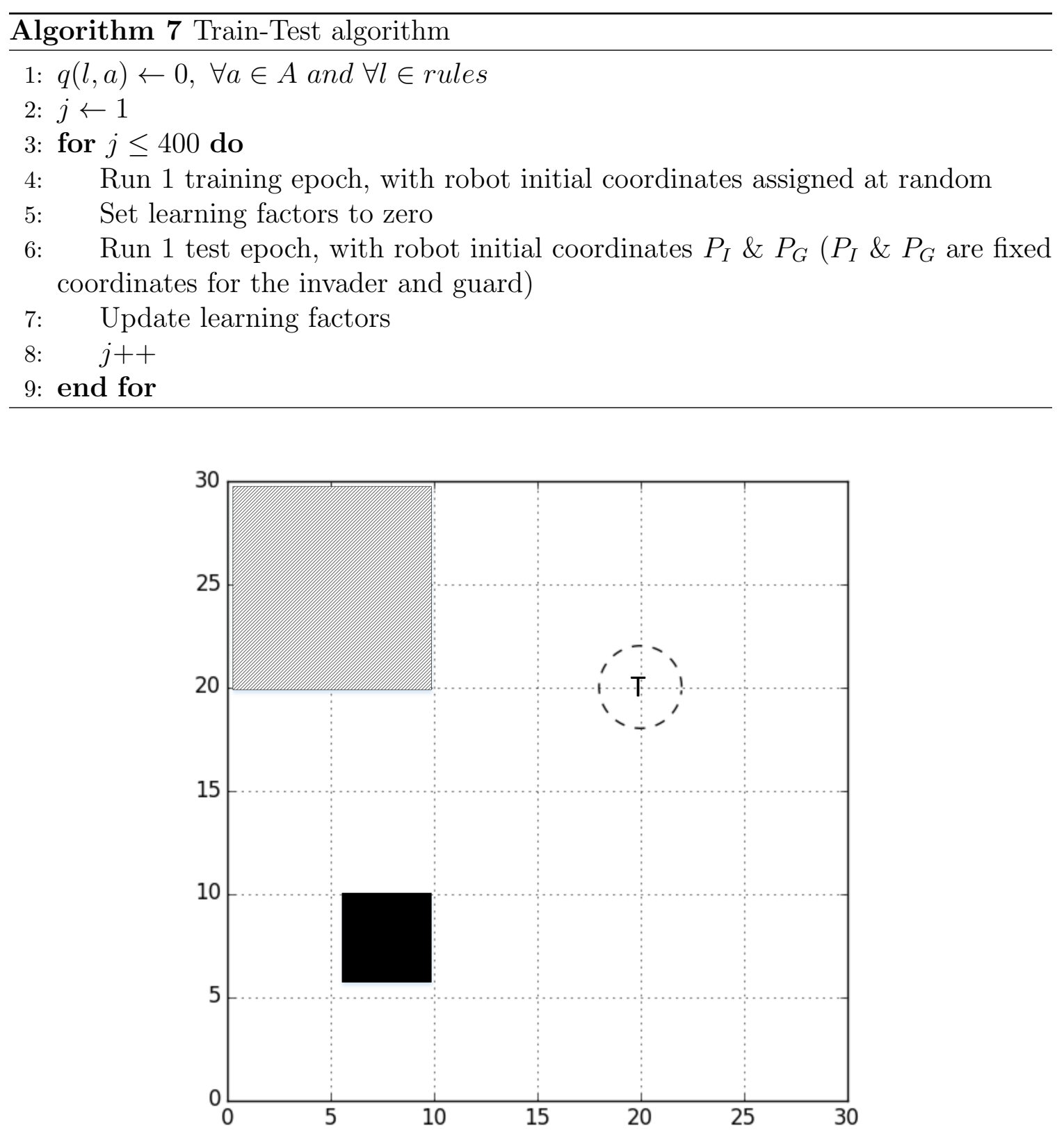

Figure 5.22: Training region

The learning parameters were chosen to decay after every learning epoch. The decay was chosen as an exponential decay as follows (where $j$ represents the epoch count):

$$
\alpha=0.99^{j} \alpha_{0}
$$




$$
\begin{aligned}
& \gamma=0.92^{j} \gamma_{0} \\
& \epsilon=0.95^{j} \epsilon_{0}
\end{aligned}
$$

With $\alpha_{0}=0.1, \gamma_{0}=0.9$, and $\epsilon_{0}=0.3$. Even though the decay is fast, the agent will still be able to adapt many of its rules. That is because the agent will observe and update the same rules multiple times in each epoch due to the agent's continuous action and small time step.

The instantaneous reward was arranged to aim at reducing the distance between the invader and the territory and increasing the distance between the invader and the guard as described in (4.27):

$$
r_{t+1}=K \delta_{I T}-J \delta_{I G}
$$

The parameter $K$ was set to a value of 2.0 and $J$ was set to a value of 1.0. The $K$ and $J$ values were chosen based on simulation. Increasing the $K$ value or decreasing the $J$ value make the invader go in a straight line towards the territory, ignoring the guard. The results examined were based only on the test epochs results.

\subsubsection{Optimal Guard Simulation Results}

The first simulation was done using the learning invader against the optimal strategy guard (described in Sec. 3.3.1). Figure 5.23 shows how the invader performs before any learning takes place. Figure 5.24 shows the invader's and guard's trajectories for the first test epoch, in this case capture of the invader. Figure 5.25 shows the the guard's and the invader's trajectories in the last test epoch after the invader learns. 


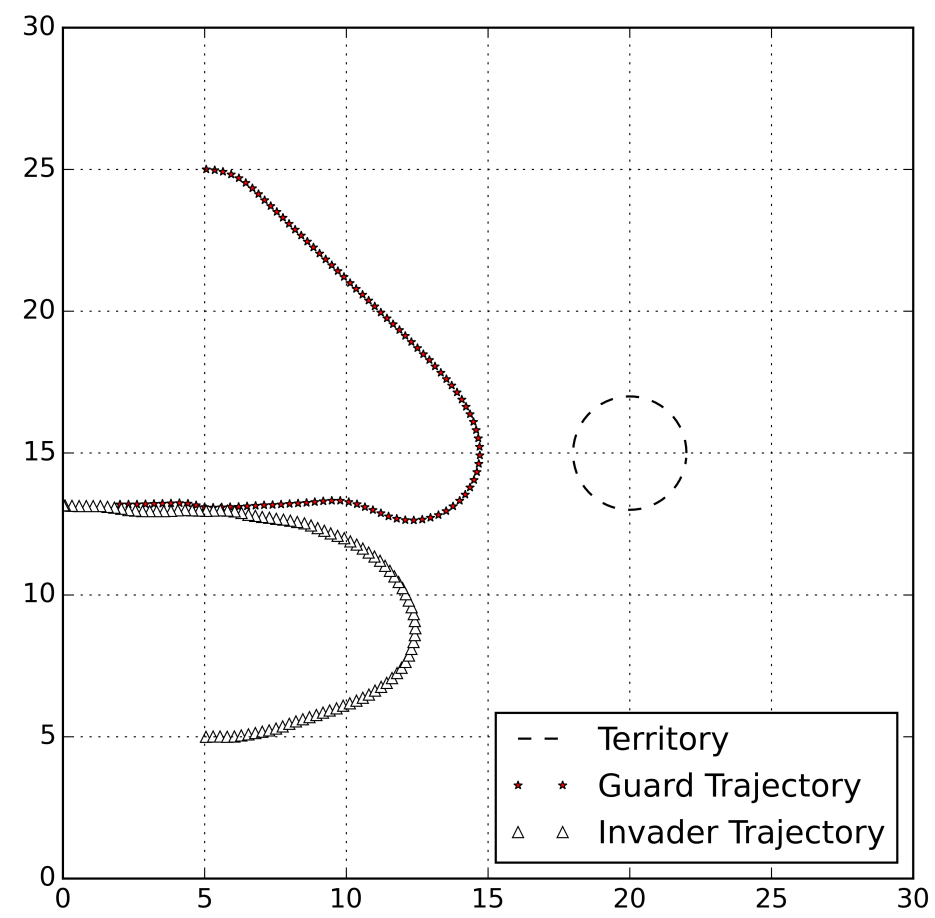

Figure 5.23: Invader vs. Optimal Guard - before learning

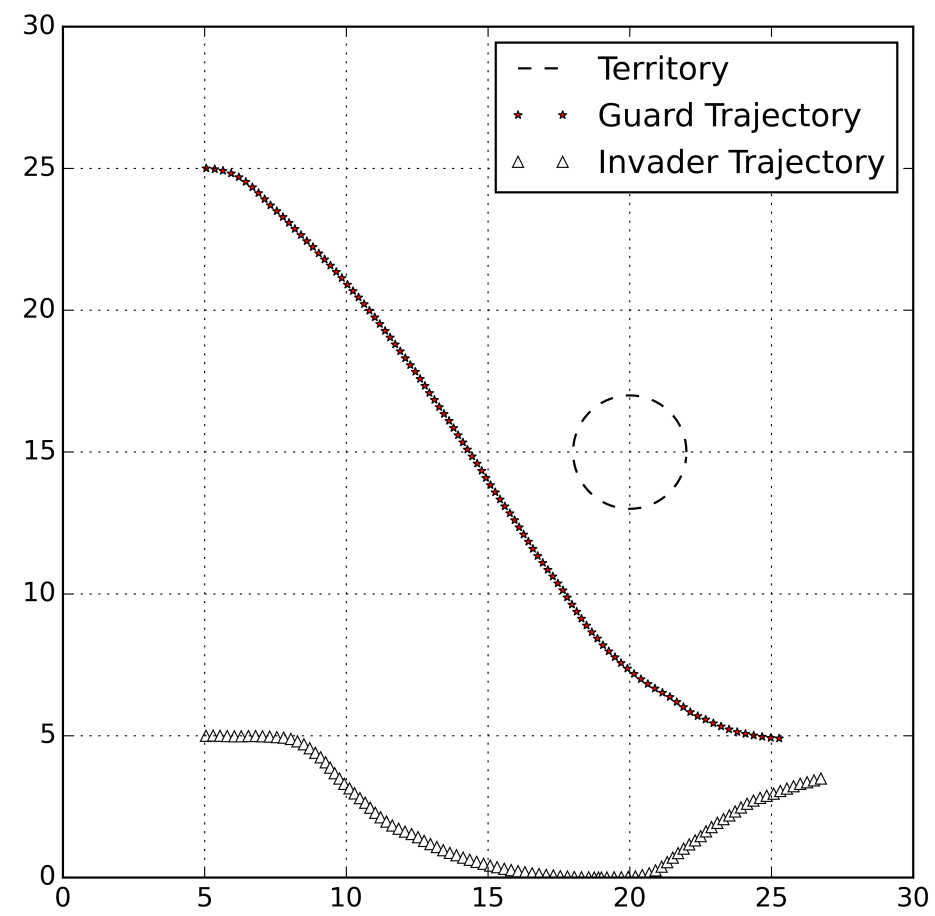

Figure 5.24: Learning Invader vs. Optimal Guard - first test epoch results 


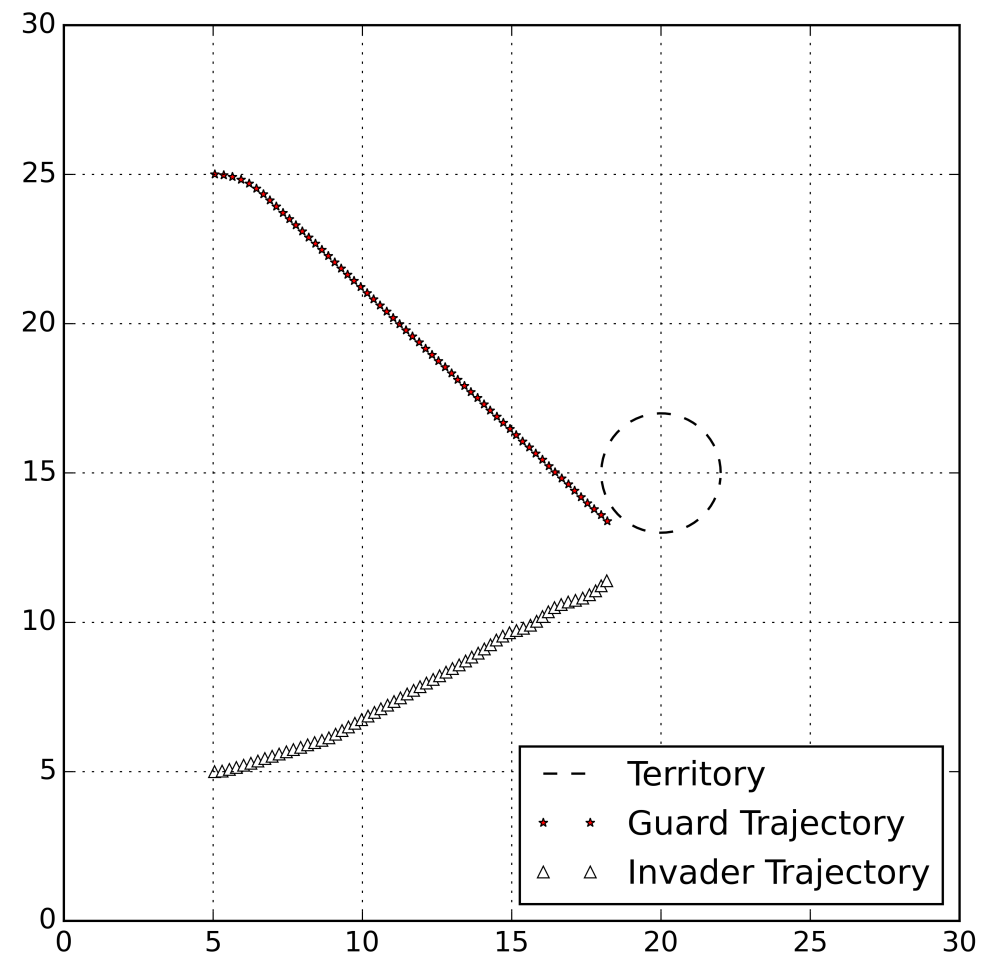

Figure 5.25: Learning Invader vs. Optimal Guard - final test epoch results

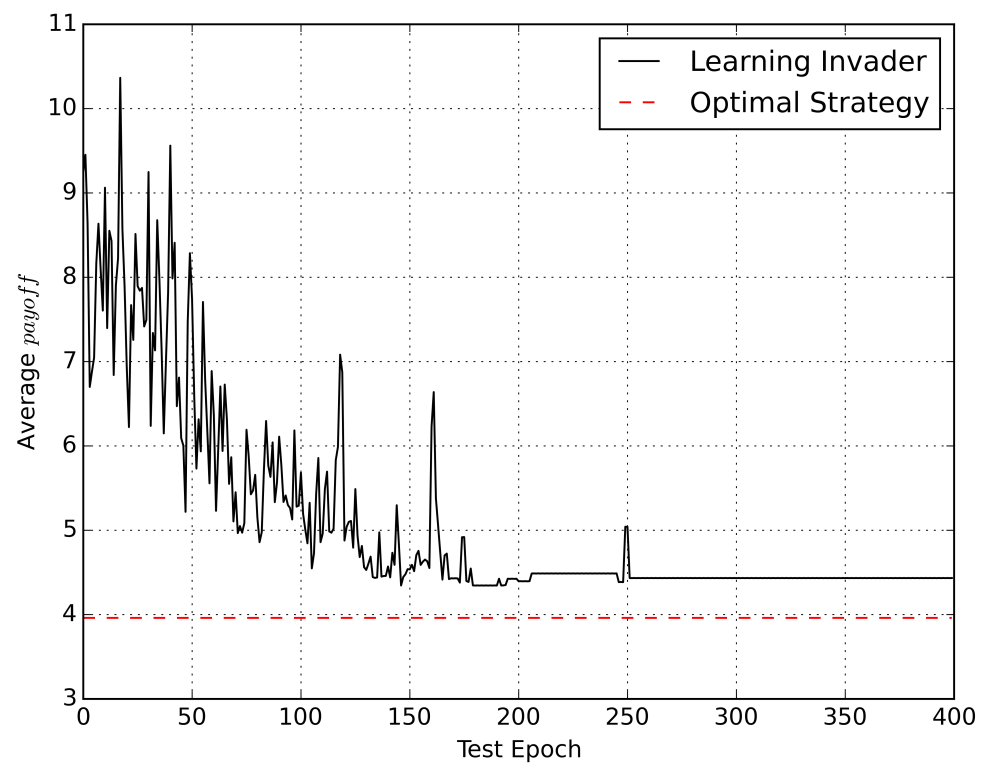

Figure 5.26: Learning Invader vs. Optimal Guard - Invader's payoff

It can be seen from Figure 5.25 that the invader was able to perform better and 
got closer to the territory. Figure 5.26 shows the average payoff of 10 simulations. It can be observed that the learning agent's payoff converged to a specific payoff value. The learning agent's average final payoff was 4.43. If the invader was following the Nash Equilibrium strategy (described in Chapter 4), it's payoff will be 3.96. The learning agent was able to get very close to the Nash Equilibrium target $(\approx 0.5$ units apart) and minimized the invader's payoff.

Once the invader notices that the guard is trying to stand between it and the territory, the invader has learnt to target the territory, since it is the best it can do.

\section{Possible Reaching}

In the simulation demonstrated in Figure 5.25 , the players' starting positions make it impossible for the invader to reach the territory (since the guard is doing the optimal strategy).

We test our invader by starting the game with positions that make it possible for the invader to reach territory. If our invader is rational, it should reach the territory.

We set the invader's starting position to $(7,7)$ and the guard's starting position to $(5,25)$. We use the Q-table that produced the results in Figure 5.25. After running the simulation epoch, we find that the invader was able to reach the territory as seen in Figure 5.27. The same simulation was repeated for the 10 different final Q-tables that produced Figure 5.26. The invader was always able to reach the territory for the different Q-tables. 


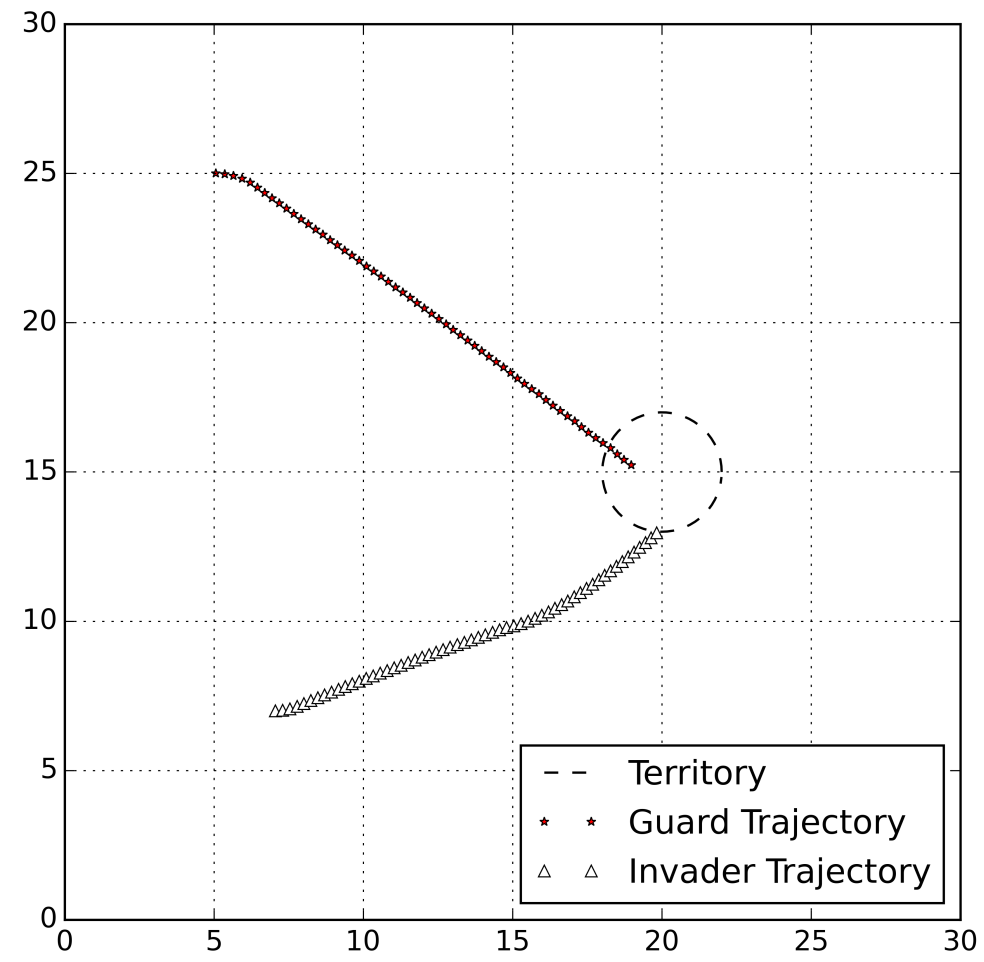

Figure 5.27: Learning Invader vs. Optimal Guard - possible reaching test

\subsubsection{Irrational Guard Simulation Results}

In our second simulation, the irrational guard algorithm (described in Sec. 3.3.2) is used instead of the optimal guard's strategy. The learning parameters (Q-table) are reset and the agent learns again. Figure 5.28 shows the test results after the first test epoch. 


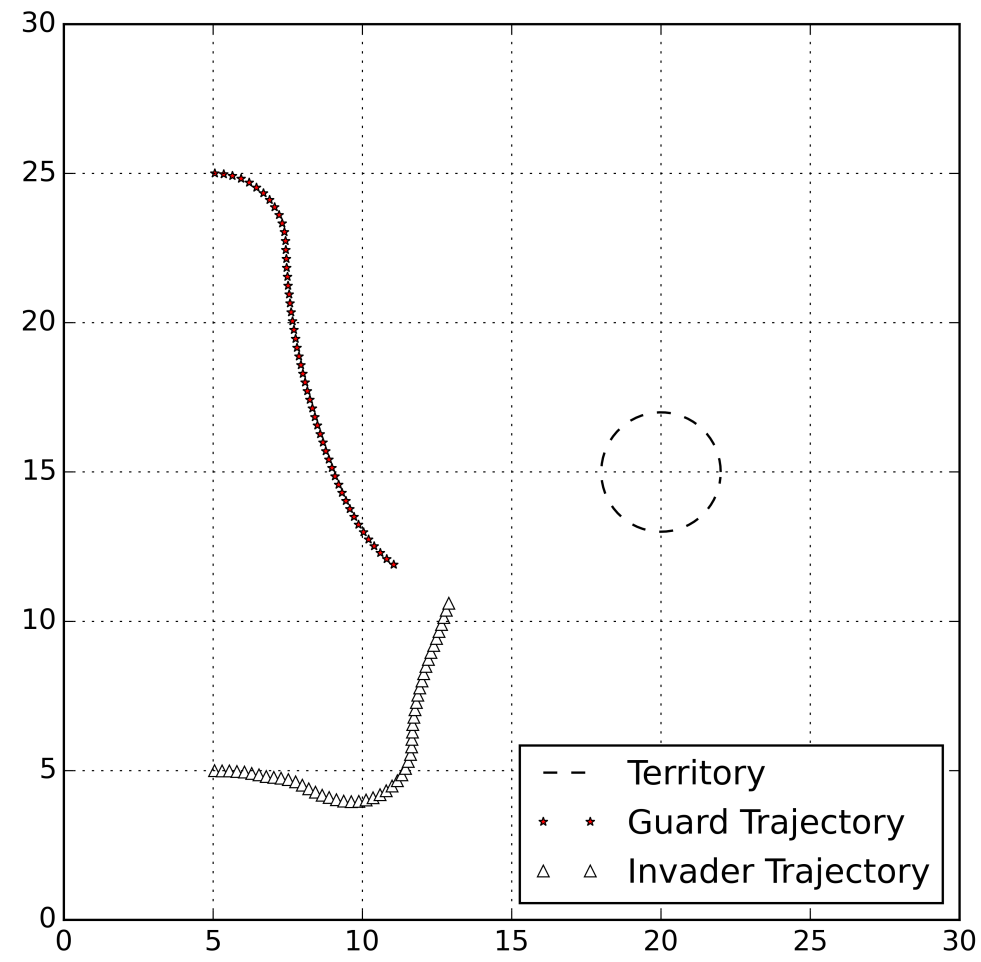

Figure 5.28: Learning Invader vs. Irrational Guard - first test epoch results

Figure 5.29 demonstrates how the invader performs in the final test epoch after learning. It can be seen how the invader was able to reach the territory due to the irrational guard's strategy. The invader's strategy became to decrease the distance to the territory, but not target it. Thus, forcing the guard to move towards the invader and to leave enough space for the invader. Then once it is clear to go to the territory, the invader makes a sharp turn and goes directly to the territory. The same simulation was run 10 times, then the simulations' payoff was averaged and plotted in Figure 5.30. 


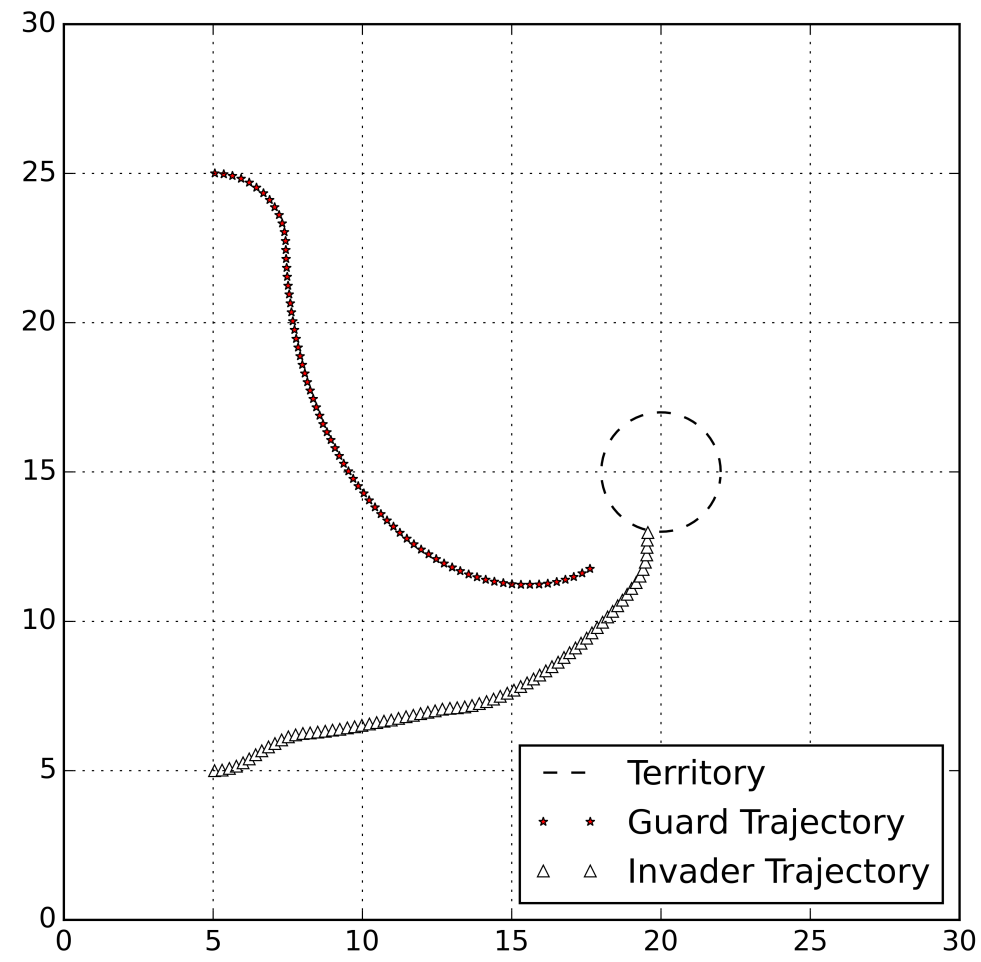

Figure 5.29: Learning Invader vs. Irrational Guard - final test epoch results

It can be seen that the agent reached the territory. To be able to compare our learning agent against the irrational guard strategy, the irrational guard strategy was run against the invader's Nash Equilibrium strategy (with no learning) to create a benchmark as shown in Figure $5.30($ payoff $=4.34)$. This shows that the invader was able to adapt and perform better than the benchmark algorithm. 


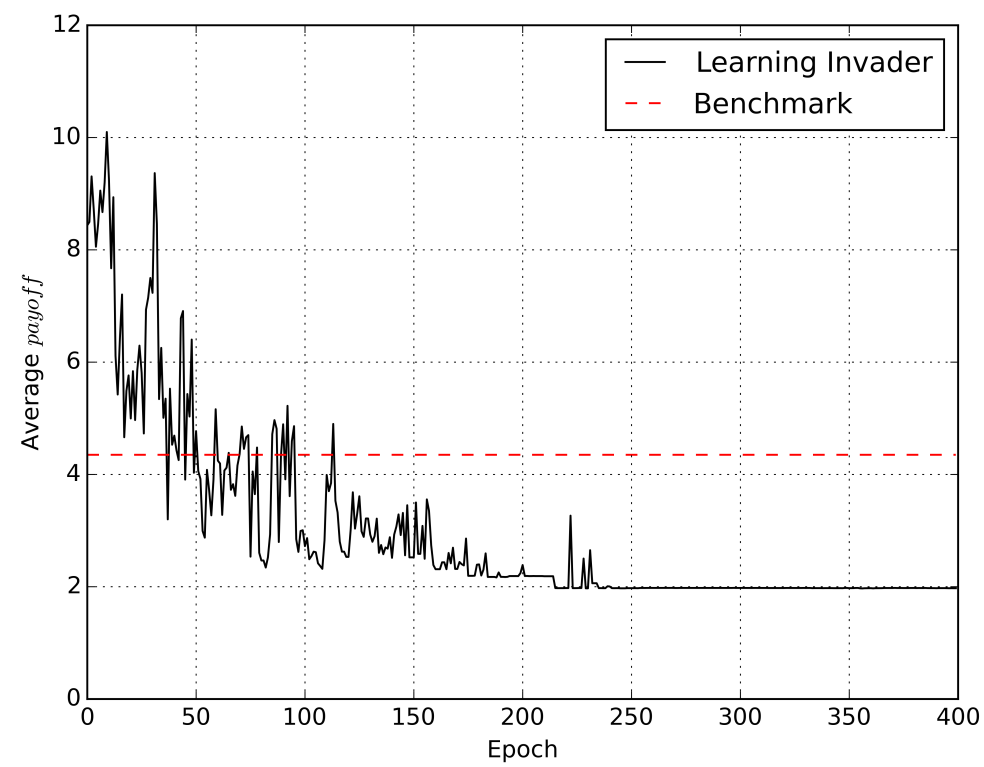

Figure 5.30: Learning Invader vs. Irrational Guard - Invader's payoff

\subsection{Review of Simulation Results}

\section{Learning Invader vs. Optimal Guard}

In this case the guard is smart and is following the optimal strategy, which is to intercept the invader and make sure it stays as an obstacle between the invader and the territory. Once the invader notices that the guard is trying to stand between it and the territory, the invader's strategy becomes targeting the territory, since it is the best it can do. Also, if there is a possibility for it to reach the territory, the invader will act rationally and reach the territory.

\section{Learning Invader vs. Irrational Guard}

The invader's strategy has learnt to decrease the distance to the territory, but not target it. Thus, forcing the guard to move towards the invader and to leave enough space for the invader. Then once it is clear to go to the territory, the invader makes 


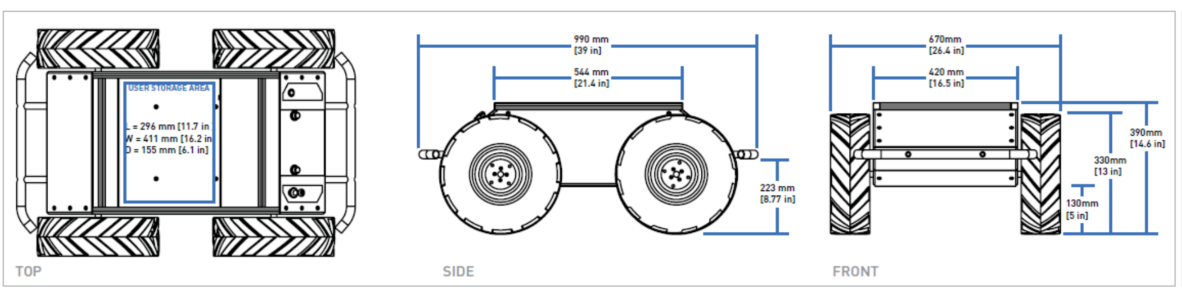

Figure 5.31: HUSKY Robot specs

a sharp turn and goes directly to the territory. In other words, the invader attracts the guard towards itself and mislead the guard with its heading. This is done until there is enough of a window for the invader to escape from the guard and reach the territory.

\subsection{Guarding a Territory game Experiment Setup}

Laboratory experiments were conducted at the Royal Military College of Canada to demonstrate how our learning agents will perform in real-life environments. CLEARPATH HUSKY robots were used as our players. These are 4 wheeled mobile robots with differential drives. The robots were programmed and configured using the Robot Operating System (ROS) and the python scripting language [50].

Each robot had its own independent software running (decentralized system). The game area was tracked using the OptiTrack system. This system consists of 24 synchronized infrared cameras. The tracking system sends the robot positions at a frequency $\approx 100 \mathrm{~Hz}$ to a central database. The robots can request that tracking information. The experiment environment is illustrated in Figure 5.32, the arrows display the possible flow of information.

Our experiments mapped the simulation size 30unit $\times 30$ unit to 8.3 meter $\times$ 


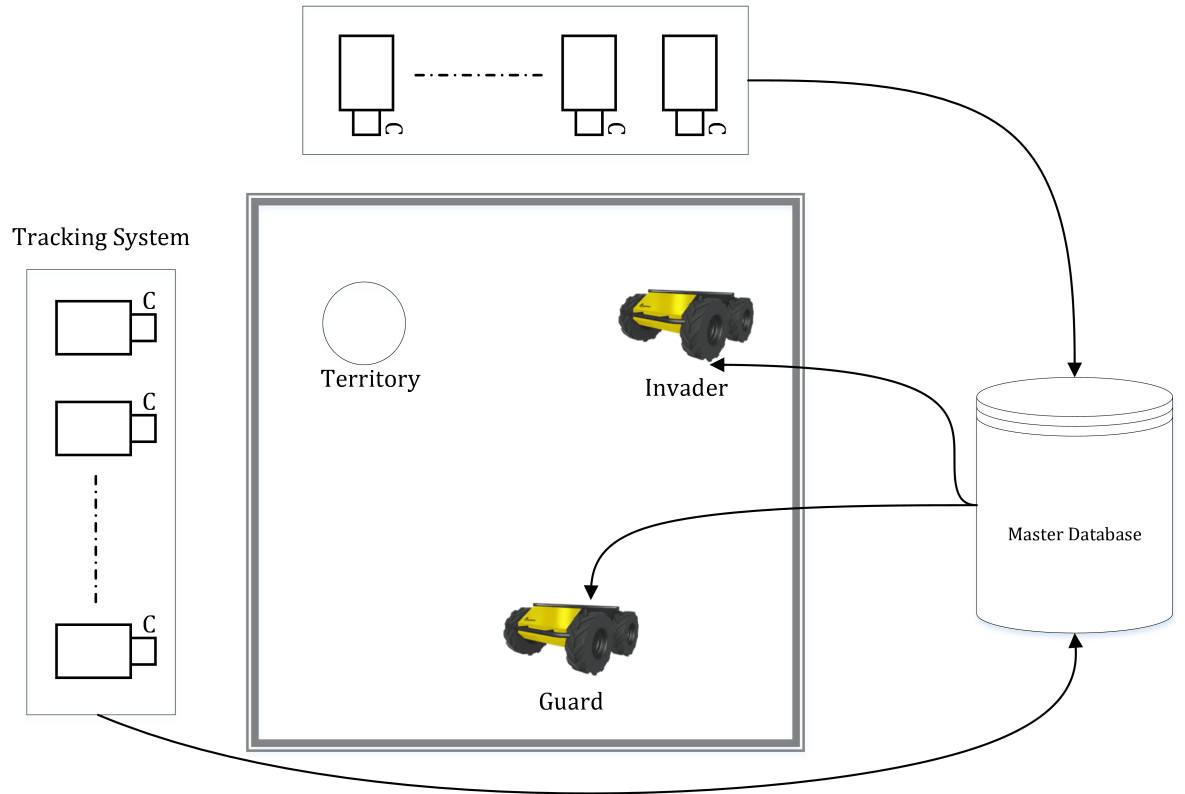

Figure 5.32: Experiment Setup

8.3meter. Players' kinematics were set such that:

$$
\begin{aligned}
& \text { Invader }: v_{I}=0.35 \text { meter } / \mathrm{sec} \\
& \text { Guard : } \quad v_{G}=0.42 \text { meter } / \mathrm{sec} \\
& \omega_{j}=-0.5,0, \text { or } 0.5 \mathrm{rad} / \mathrm{sec}
\end{aligned}
$$

It was difficult to always make the robots start at exactly the same position.

\subsection{Experimental Evaluation of Simulation Re- sults}

In this section, we evaluate the invader's performance using the Q-table deduced from the simulations. In other words, we are going to test our numerical results (from the simulations) in a real-life scenario. The invader uses the learnt parameters from the 
simulations (the Q-table). There is no learning while the experiment is running. We use the Q-table from the final simulation epochs demonstrated in Sec. 5.3 .

\section{Learning Invader vs. Optimal Guard}

In this experiment we use the Q-table used in the simulation demonstrated in Sec. 5.3.1. The first experiment trajectory results can be seen in Figure 5.33.

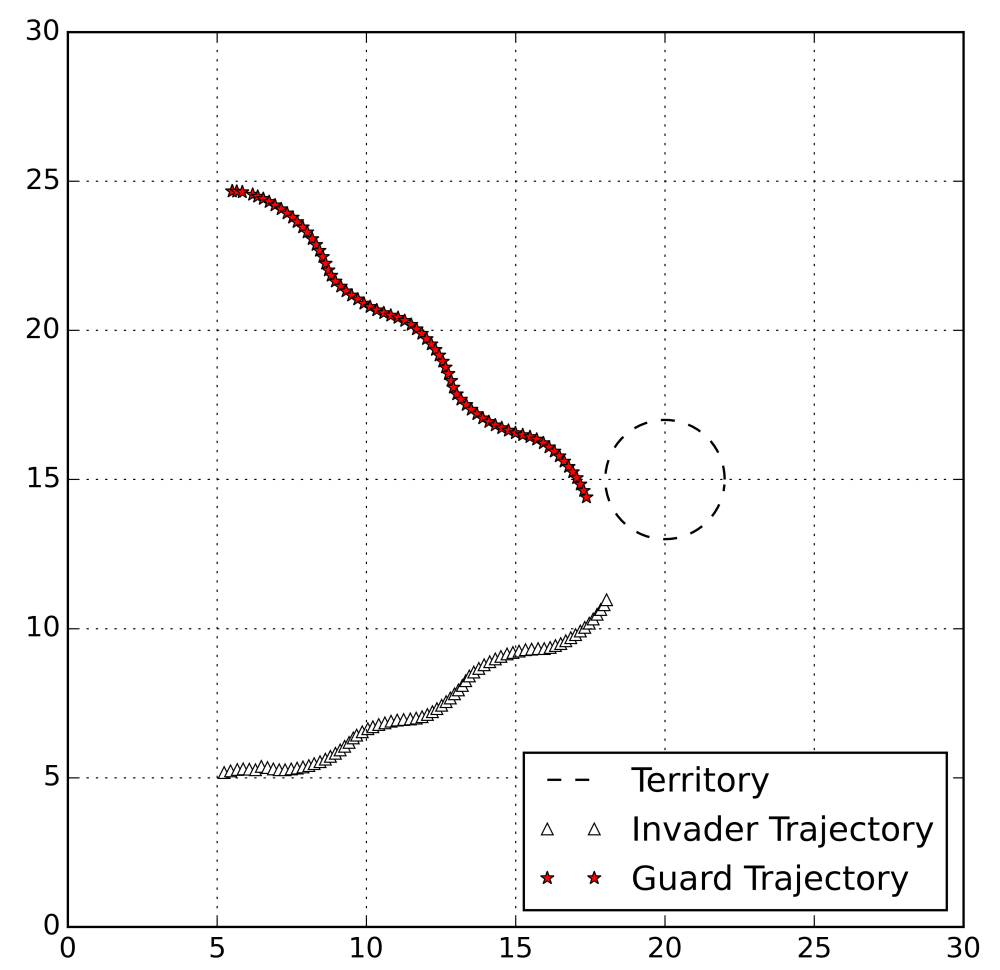

Figure 5.33: Experiment Trajectories: Learning Invader vs. Optimal Guard

The same experiment was run 10 times. The invader's payoff average was 4.7 with standard deviation of 0.5 .

\section{Learning Invader vs. Irrational Guard}

In this experiment we use the Q-table used in the simulation described in Sec. 5.3.2. The experiment trajectory results can be seen in Figure 5.34 . 


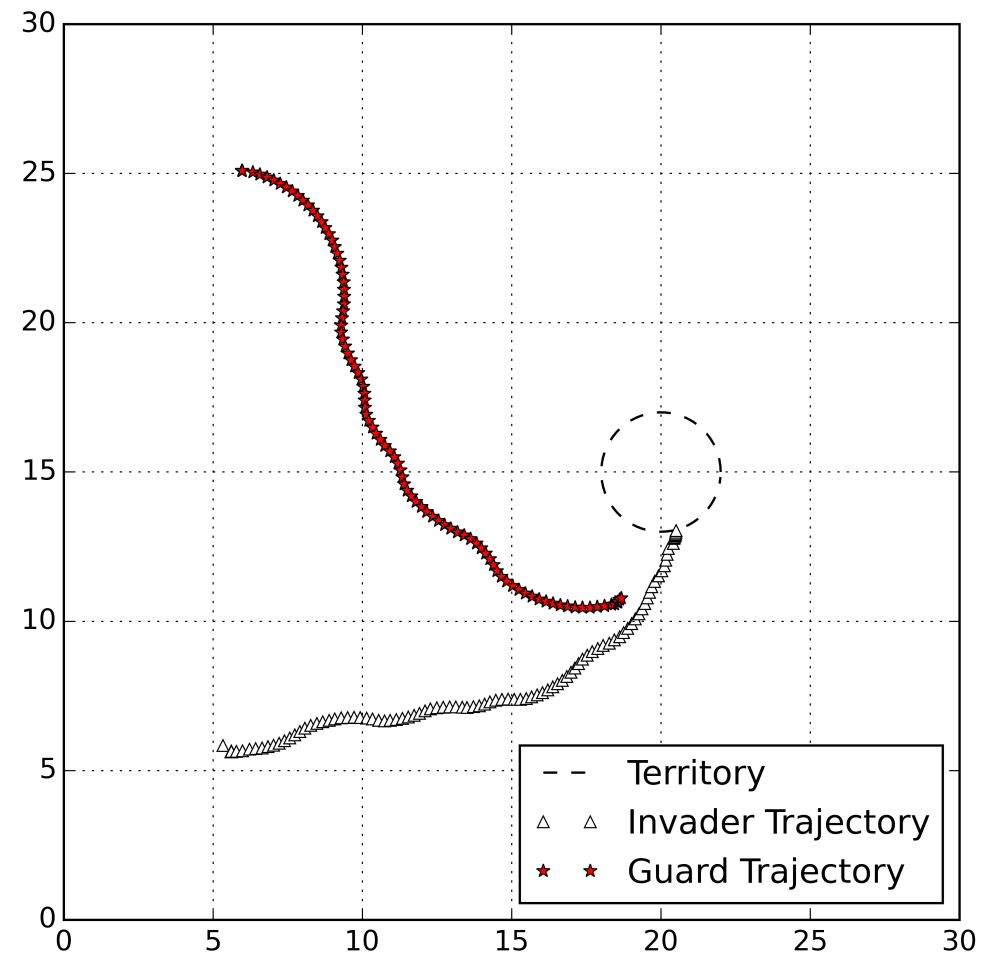

Figure 5.34: Experiment Trajectory: Learning Invader vs. Irrational Guard

The same experiment was run 10 times. The invader was always able to reach the territory (similar to the simulations).

\section{Benchmark: Optimal Invader vs. Optimal Guard (no learning used)}

This experiment describes when both players have a fixed algorithm. Both players will follow the optimal solution and target the closest interception point to the territory. The fixed algorithms result in the Nash Equilibrium described in Sec. 3.3. No learning was used to produce these results. The experiment trajectory results can be seen in Figure 5.35 . 


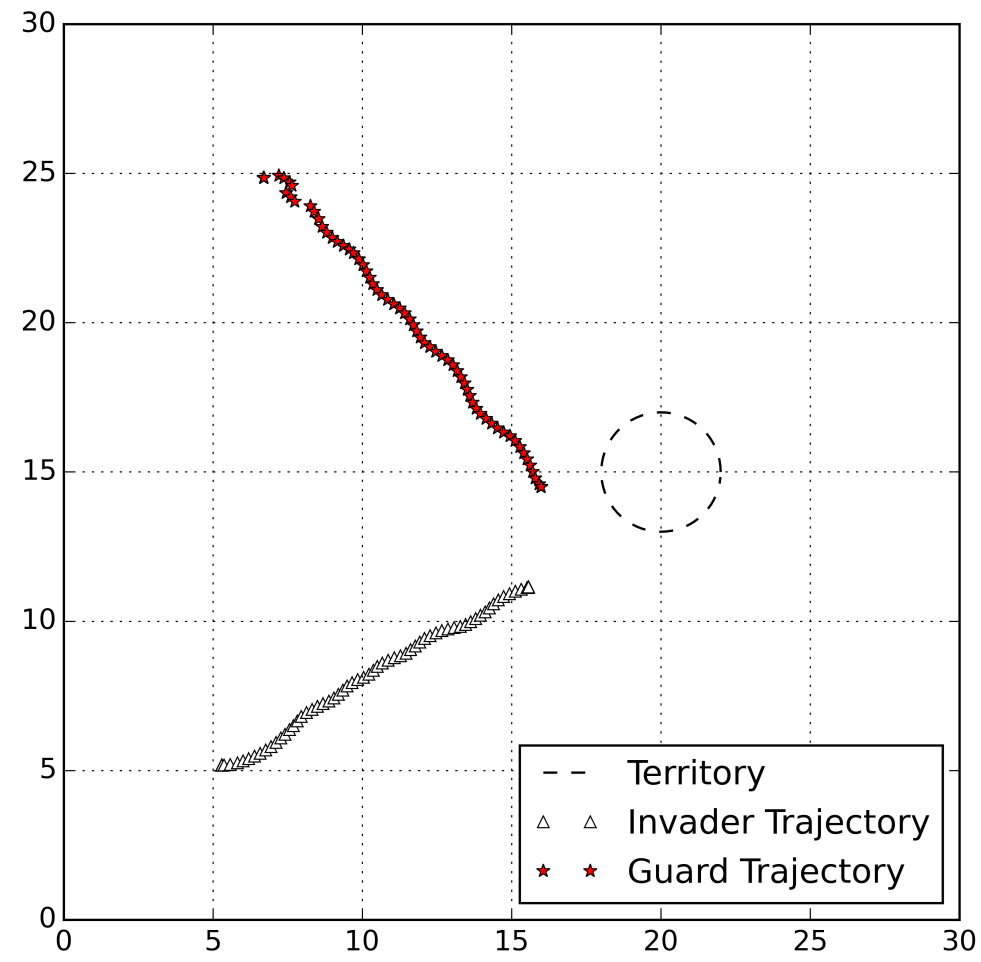

Figure 5.35: Experiment Trajectories: Both players are following Nash Equilibrium

The same experiment was run 5 times. The invader's average payoff was 5.3 with standard deviation of 0.9units.

Benchmark: Nash Equilibrium strategy Invader vs. Irrational Guard (no learning used)

This experiment describes when both players have a fixed algorithm. The guard will use the irrational guard algorithm and target the invader. The invader will use the Nash Equilibrium strategy. This was our benchmark results in Sec. 5.3.2. No learning was used to produce these results. The experiment trajectory results can be seen in Figure 5.36 . 


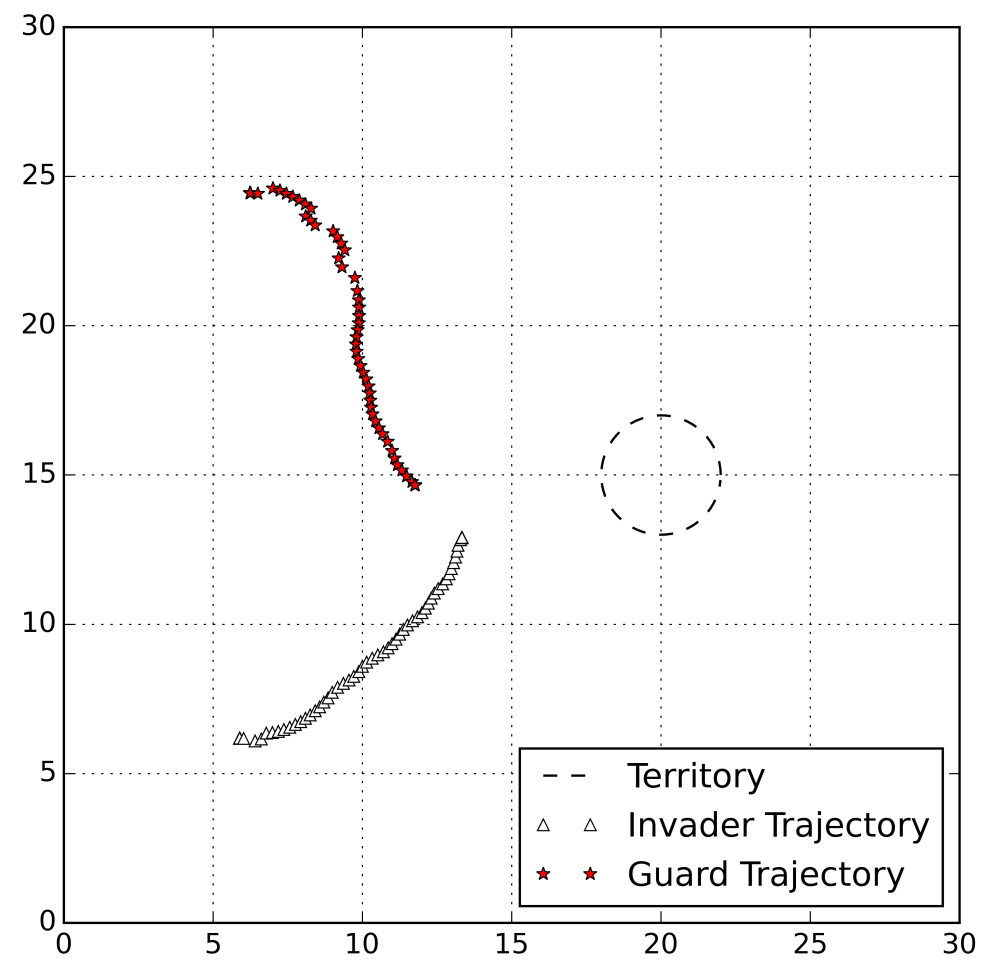

Figure 5.36: Experiment Trajectories: Irrational Guard vs. Invader doing Nashstrategy for an optimal guard

The same experiment was run 5 times. The invader was unable to reach the territory. The invader's average payoff was 6.3 with standard deviation of 0.6.

\subsection{Online Learning}

In online learning, the learning agent adapts its Q-table as it interacts with the environment (as it plays the game). The Q-table is first initialized to zeros. The agent uses the learnt parameters from the previous experiment(s) at the start of its new experiment. We always let the invader start the experiment at coordinate $(5,5)$ and the guard at $(5,25)$.

Each experiment represents a game run until termination time. The payoff is recorded after each experiment. The experiment terminates in any of the following 
conditions:

- The guard captures the invader

- The invader reaches the territory

- Either of the players enters a blind region and can not be tracked

Even though most of the experiment environment was tracked using The OptiTrack system, some regions had lower tracking precision causing blind regions. The blind regions were mostly near the corners and edges. Figure 5.37 demonstrates a rough estimate of the trackable regions as the shaded region.

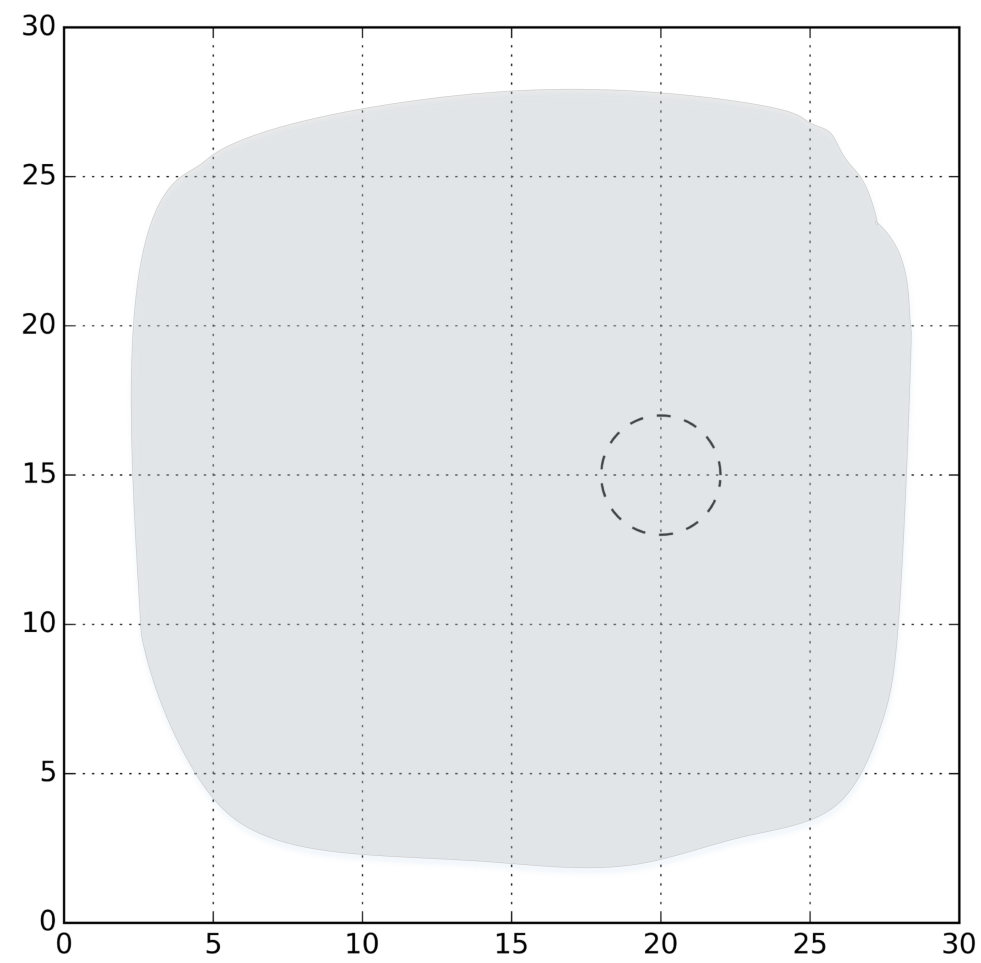

Figure 5.37: Experiment Environment: trackable region

We run two different sets of experiments to demonstrate how the invader learns online versus the different guard strategies. 


\section{Learning Invader vs. Optimal Guard}

After programming the guard to follow the optimal strategy, we ran 100 experiment.

In each experiment, the invader used the parameters learnt from the previous experiment. In this experiment we are not expecting the invader to reach the territory, since the guard is following the optimal strategy.

The first experiment trajectories can be seen in Figure 5.38 where the invader performed poorly and entered a blind region. Figure 5.39 displays the players trajectories after learning. The payoff of each experiment was recorded and plotted in Figure 5.40 , This figure also demonstrates how our invader's strategy evolved and minimized the invader's payoff. In the last 20 experiments in online learning, the agent produced an average payoff of $3.82 \pm 0.23$. This is better than the output from experimental evaluation of simulation results illustrated in Figure 5.33. The agent adapted to the physical realities of the experimental environment. 


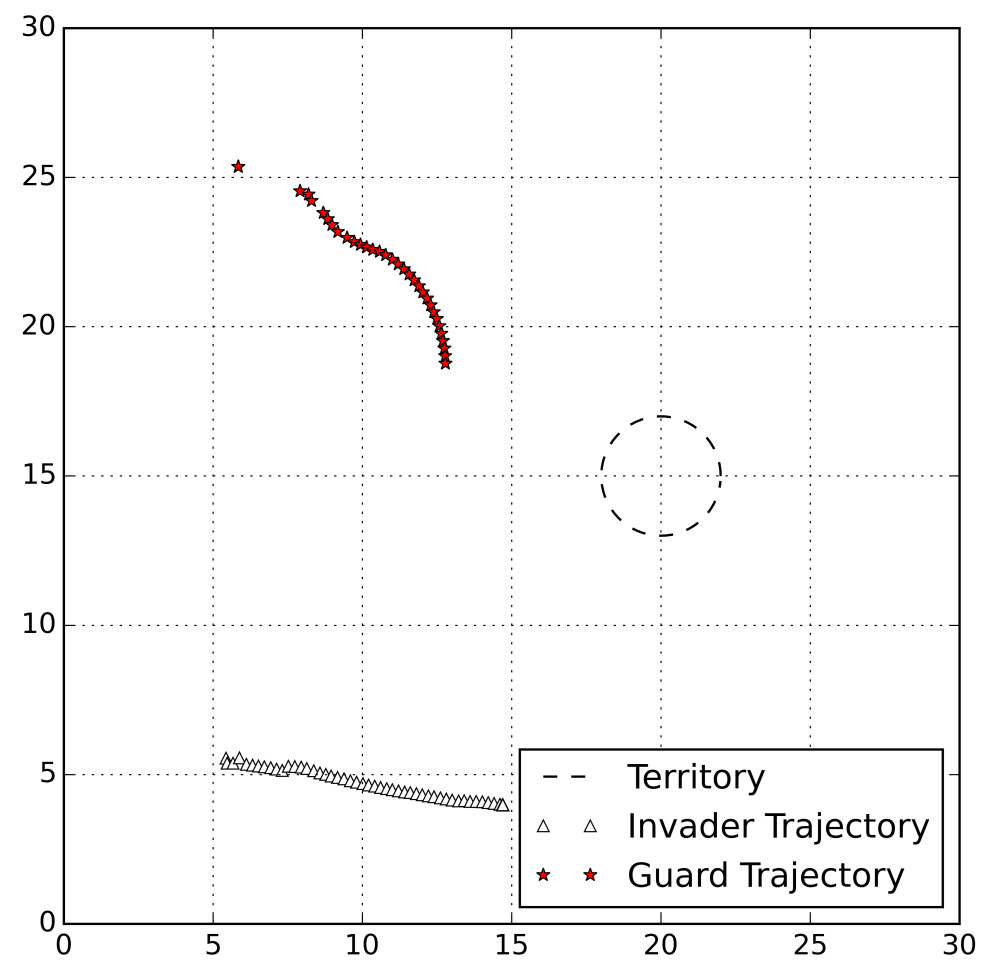

Figure 5.38: Online Learning: Learning Invader vs. Optimal Guard - first experiment 


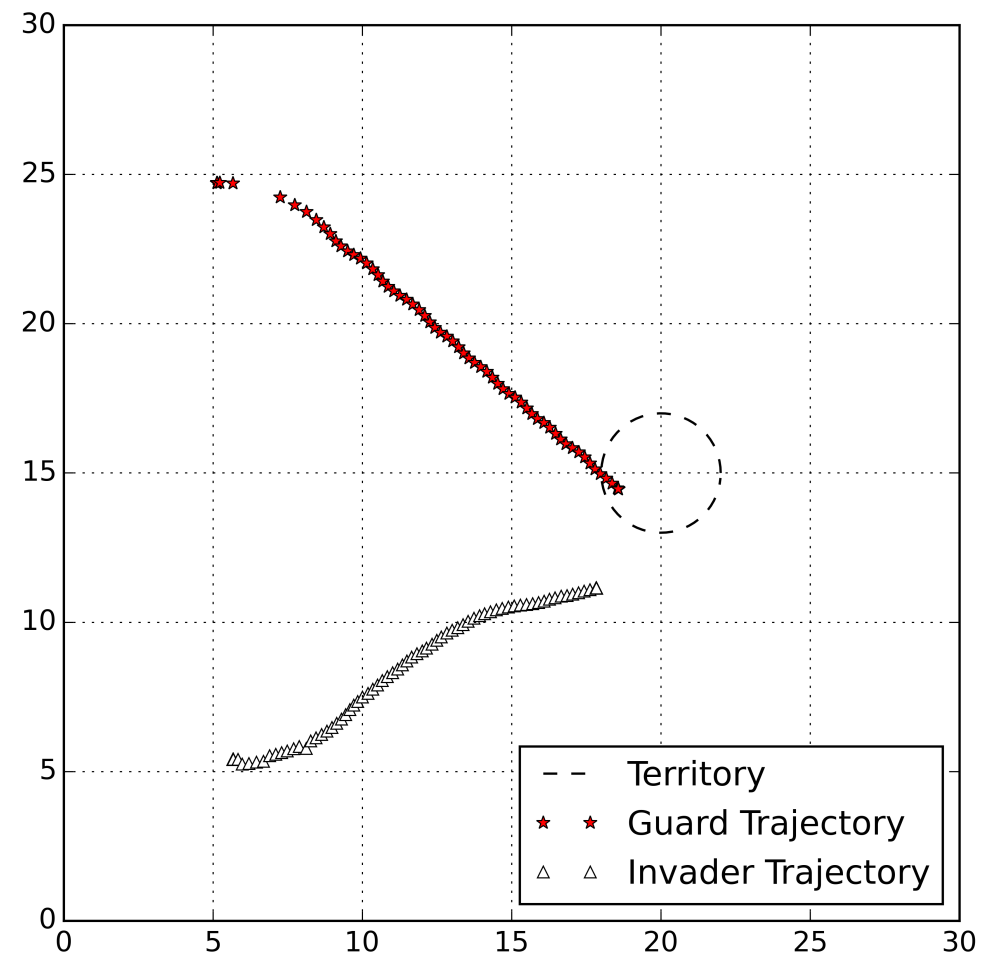

Figure 5.39: Online Learning: Learning Invader vs. Optimal Guard - Last experiment

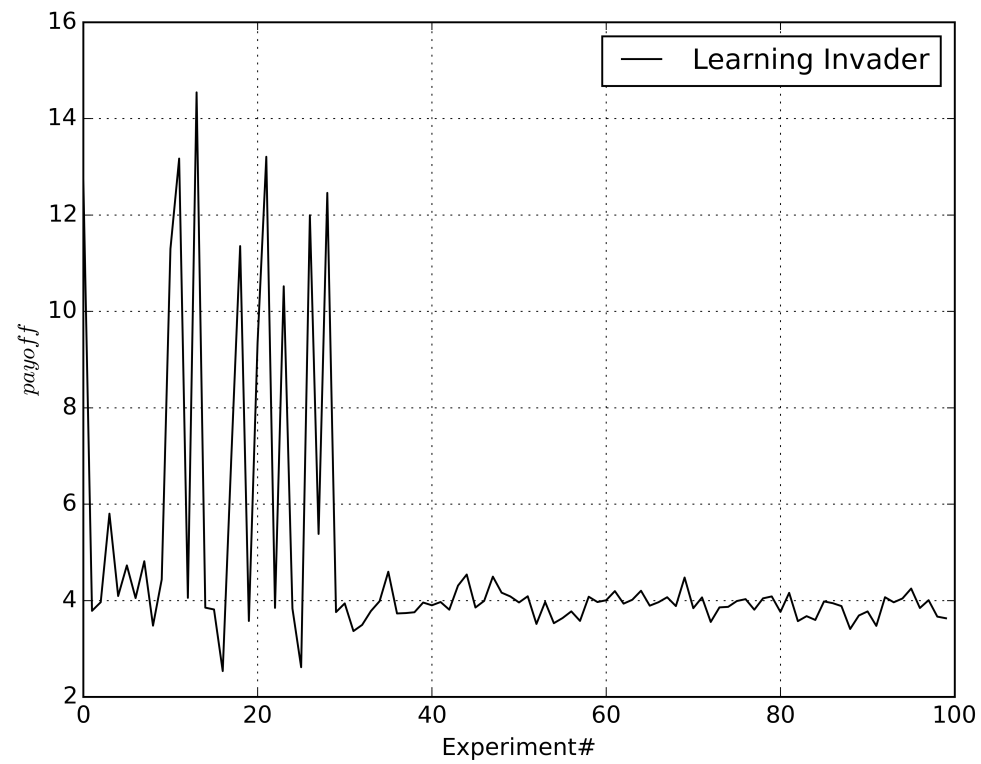

Figure 5.40: Online Learning: Learning Invader vs. Optimal Guard - payoff 


\section{Learning Invader vs. Irrational Guard}

After programming the guard to follow the irrational strategy, we ran 50 experiments.

The first experiment trajectories can be seen in Figure 5.41, where the invader was capturerd. Figure 5.42 displays the players' trajectories after the learning took place. The payoff of each experiment was recorded and plotted in Figure 5.43. We stopped at 50 experiments since the invader was able to reach the territory more than 10 times in a row. Figure 5.43 demonstrates how our invader's payoff converged into reaching the territory. Furthermore, the agent once again adapted to the physical realities of the experimental environment and reached the territory in a shorter time than in illustrated in Figure 5.34 .

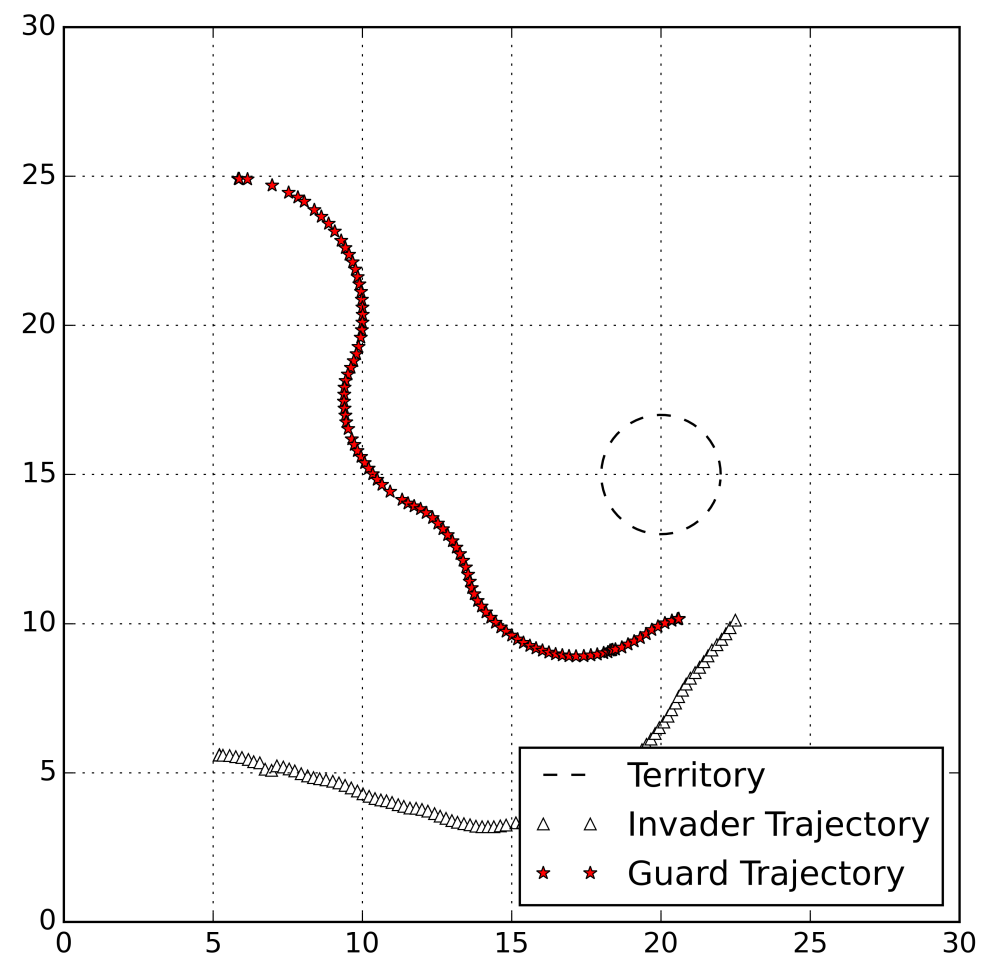

Figure 5.41: Online Learning: Learning Invader vs. Irrational Guard - first experiment 


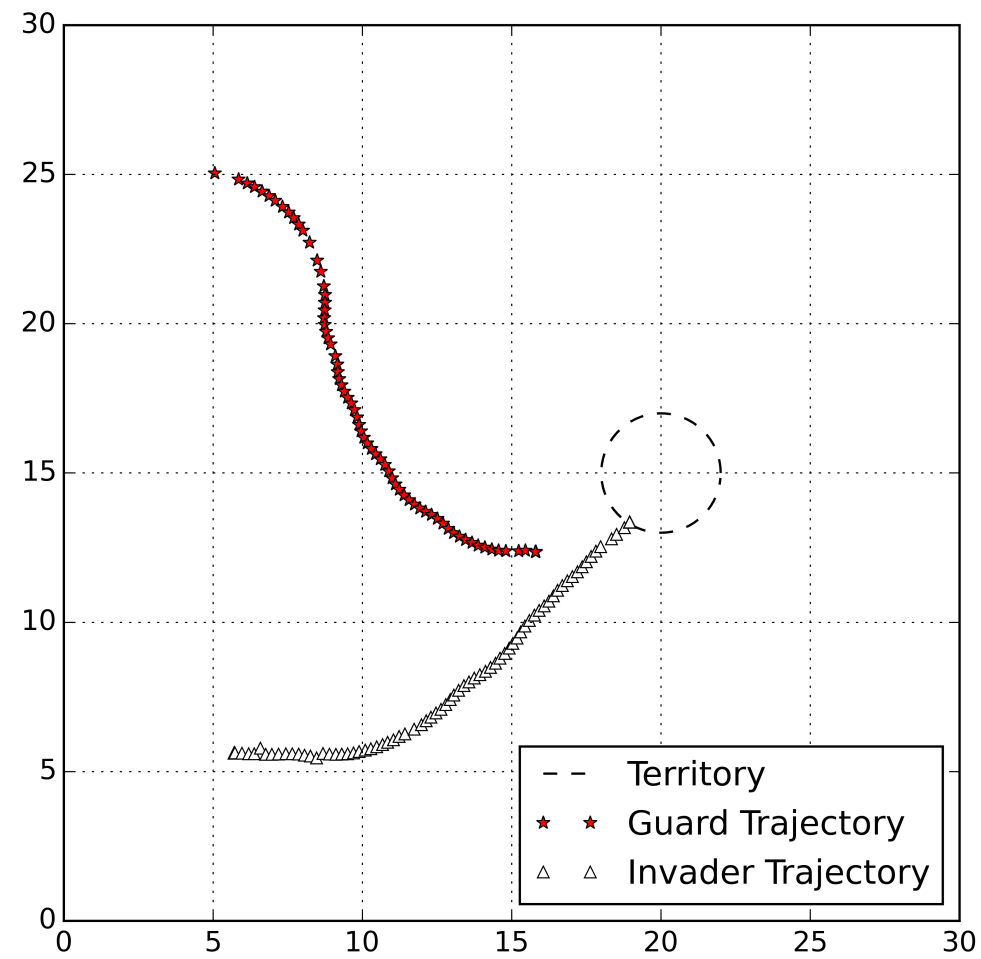

Figure 5.42: Online Learning: Learning Invader vs. Irrational Guard - Last experiment

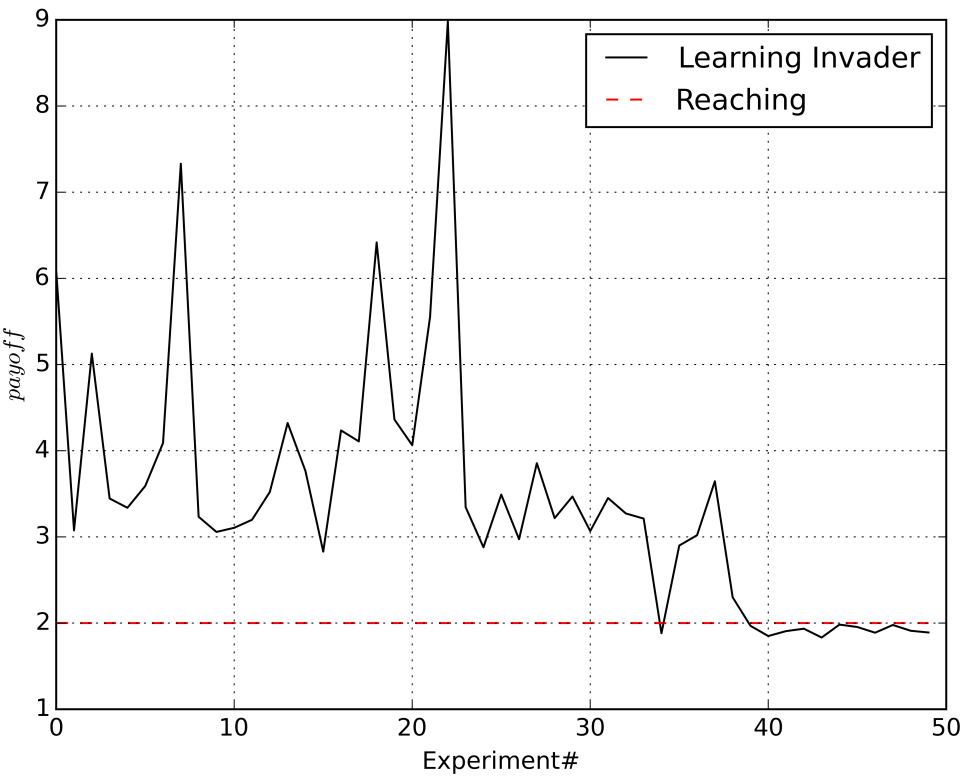

Figure 5.43: Online Learning: Learning Invader vs. Irrational Guard - payoff 


\subsection{Discussion and Summary}

In the grid world simulation, we were first able to demonstrate that the learning agents were able to converge to Nash Equilibrium using two different algorithms; the Minimax Q-Learning and the WoLF-PHC algorithms. Our results demonstrated that WoLF-PHC has slower convergence rate when both players are learning. Furthermore, the results demonstrated that if one player is following a fixed strategy, the other player (learning) will converge into another pure strategy that will maximize the learning agent's rewards. These results are similar to our expected results from theory as described in Chapter 2 .

Second, we started our simulations to demonstrate the use of Reinforcement Learning in the differential game. Simulations started with a simple game (single-player). This was done to demonstrate the differences between the FQL and the FACL algorithms. This was also done to compare the performance of one of our controller inputs to the controller performance described in [5]. Both learning algorithms converged such that the invader was able to reach the territory. However, the FQL produced superior results due to its usage of the action set $A$ unlike the FACL algorithm. The FACL algorithm will become more useful when no information about the action space is known. We also demonstrated that using a single input $\theta$ for this version of the game performed better than the controller inputs used by [5] with our non-holonomic model. The FQL algorithm adapted faster and led the invader to choose the shortest path to the territory.

Third, we implemented the FQL algorithm on the learning invader for the guarding a territory game. Simulations were run having the guard follow different strategies. Against a guard employing an optimal strategy, the invader was able to get very close to the Nash Equilibrium point $(\approx 0.5$ units). 
In the second simulation, the invader competed against an irrational guard's strategy. Following a Nash Equilibrium strategy against an irrational guard would be a bad idea since the guard is behaving differently than the optimal. It was seen from the second simulation that the agent was able to adapt to the new guard strategy. It also performed better than the invader's Nash Equilibrium strategy. The agent's strategy converged into reaching the territory in this case. This shows that our learning agent was able to adapt and adjust its strategy to perform best with the current guard's strategy.

Our training method, focused on specific regions for both players. Other training methods can also be considered to optimize the agent's performance in any position in the game. This can be done by using a different training region as described in Figure 5.44. Such that the shaded circle describes the pool for the initial training positions for both players. The players can pick a random position from that region. However, training will take a longer time in this case and the learning rates need to be adjusted differently. The parameters will have to then decay slower to ensure learning in different configurations. 


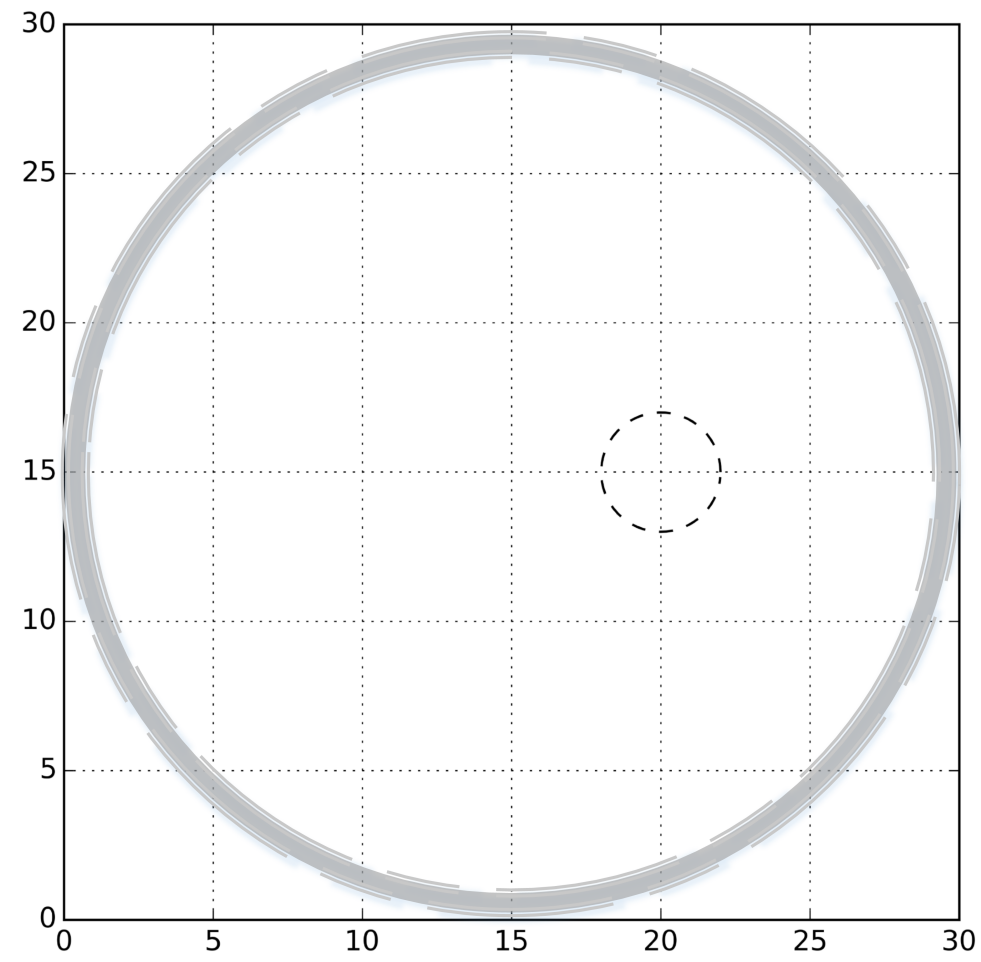

Figure 5.44: Alternative Training Region

Fourth, we ran experiments to evaluate the numerical results produced in the simulations. This was done to verify that our simulation model was close to reality. Results seen in the experiments for the learning invader were almost identical to simulation results. However, the optimal fixed algorithm followed by the invader did worse than expected. This is probably due to the algorithm not being able to handle noisy information and time delays between the time it received the information and time it acted on it and model mismatch.

Fifth, we were able to run online learning experiments. The learning agent did not use simulations to adapt its parameters. The invader adapted its parameters through interacting with the real-life environment.

When the invader ran against the optimal guard strategy, it adapted its parameters and performed better than in the experiments that used the simulations' output. 
Such that: its average payoff in the last 20 online-learning experiments was 3.82 with 0.23 standard deviation. The average payoff for simulation experimental evaluation was 4.7 with standard deviation of 0.5 .

When the invader ran against the irrational guard strategy, it adapted its parameters within 50 experiment and was able to reach the territory. 


\section{Chapter 6}

\section{Conclusion and Recommendation}

\subsection{Conclusion}

The work presented in this thesis has investigated and demonstrated the use of reinforcement learning in the guarding a territory game. Our main goal was to create and combine a customized control system with reinforcement learning to produce a learning invader agent able to play the guarding a territory game against opponents with different strategies. We were able to create a fuzzy logic controller system and combine it with reinforcement learning to produce our FQL algorithm.

Chapter 5 demonstrated simulations that showed that our customized control system made the invader able to get very close to the Nash Equilibrium when the opponent (guard) is playing the Nash Equilibrium strategy. The simulations also demonstrated that when the opponent is no longer following the Nash Equilibrium strategy, the invader was able to converge to a new strategy to maximize its rewards. Our simulations demonstrated that our learning agent was able to adapt in different game configurations against a superior (faster) guard.

Chapter 5 also demonstrated experimental evaluations of the learnt parameters from the simulations. Experimental results with the learning agent were very close 
to the simulation results. The invader (robot) was able to reach the territory when it competed with an irrational guard. The invader robot produced average payoff of 4.7 with standard deviation 0.5 when it ran against an optimal guard (average payoff was 4.43 in simulations).

We also demonstrated our online learning in Chapter 5, where the invader robot was learning as it played the game in real-life. Online learning experimental results were superior to that of simulation experimental evaluation. Against a guard employing an optimal strategy, our invader reached an average payoff of 3.82 with 0.23 standard deviation in its last 20 experiments (average payoff from simulations was 4.43). Against a guard employing an irrational strategy, our invader reached a payoff of less than two units (reached the territory) in its last 10 experiments (similar to simulations). The experiments showed that our simulations modelled the real-life scenario appropriately and that our invader performed rationally in the real-life environment. The agent adapted to the physical realities of the experimental environment.

In conclusion, the simulation and experimental results demonstrated that the agent (invader) using a customized control system (to estimate the game status) was able to adapt and perform optimally against different guard's strategies and behave as a rational invader.

\subsection{Contribution}

In summary, this thesis contributed in the following way:

1. Customizing a fuzzy control system that enables the invader to estimate the game state using 3 inputs and to pick a heading. This was described in Chapter 4

2. Proposing an instantaneous reward function that aims at decreasing the distance between the invader and the territory, and that will control the rate of decrease 
in distance between the invader and the guard.

3. Evaluation of the performance between FQL and FACL algorithms for a 1-player version of the game with non-holonomic constraints (using different controller inputs). This was done using simulations in Chapter 5 . The simulations demonstrated that the invader is able to reach the territory after learning using either the FQL or the FACL algorithms. However, it showed faster convergence using the FQL algorithm.

4. Evaluation of our customized controller with the FQL algorithm for the guarding a territory game using different guard's strategies. This was demonstrated in our simulations in Chapter 5 . The results demonstrated that the agent was able to adapt against different guard strategies. Furthermore, the invader behaved rationally.

5. Evaluation of the simulations' results through experiments using the husky robots demonstrated trajectory results very similar to our simulations. This shows that our simulation model did a good job in modelling reality.

6. Implementation of online learning experiments, such that our learning agent was able to adapt its parameters online as it interacted with the environment. This demonstrates the ability of our control system to perform well in a real-life environment.

\subsection{Recommendation for Future Work}

The work presented in this thesis can always be further expanded. There are so many applications for guarding a territory game in real-life scenarios.

The following list will describe some possible future work: 
1. More Players: The game currently consists of one guard and one invader. It will be interesting to increase the number of players (guards and/or invaders). This will mean the control system inputs will need to change. If only guards are added, the number of inputs will then increase linearly for the single invader. However, it may be better to come up with a different methodology to estimate the game state and use less number of inputs. On the other hand: if the number of invaders increased, it will be interesting if the invaders can collaborate against the guard.

2. Personality Traits: Personality Traits can enable the agent to demonstrate personalities such as bravery, cowardness, or fear. Personalities are defined with functions and variables. It will be of interest to see if the robots are able to use personality to decide on their actions. Assuming only the invaders have personalities, a possible scenario would be an invader approach the guard but only to distract him from the other invader(s).

3. Generalized Training: Most of the training done in this thesis was focused on a specific area. It will beneficial to maximize the training region and include more possibilities.

4. Limited Information: Another area for future work, that can be challenging, is to deal with limited information. The limited information could be lack of other robots positions, as well as, accurate kinematics information.

5. Extra Dimension: Currently the investigated game occurred in a 2-dimension. Current and future development have lots of interest in flying drones. Hence, it will become useful to add a 3rd dimension space and simulate and experiment on flying drones. 
6. Obstacles Avoidance: The current game environment did not contain any obstacles, but only a border. Adding static or dynamic obstacles will be a challenging task to do. The obstacle avoidance controller has to work collaboratively with the learning control.

7. Open Space Simulation: Instead of playing inside a bounded space, a more difficult case would be working in an open space. This will need the control system to use the inputs differently. 


\section{List of References}

[1] R. Isaacs, Differential Games: A Mathematical Theory with Applications to Warfare and Pursuit, Control and Optimization. 1999.

[2] W. Rzymowski, "A problem of guarding line segment," in Decision and Control, 2009 held jointly with the 2009 28th Chinese Control Conference. CDC/CCC 2009. Proceedings of the 48th IEEE Conference on, pp. 6444-6447, Dec 2009.

[3] Y.-S. Lee, K.-H. Hsia, and J.-G. Hsieh, "A problem of guarding a territory with two invaders and two defenders," in Systems, Man, and Cybernetics, 1999. IEEE SMC '99 Conference Proceedings. 1999 IEEE International Conference on, vol. 3, pp. 863-868 vol.3, 1999.

[4] X. Lu and H. M. Schwartz, "An investigation of guarding a territory problem in a grid world," in American Control Conference (ACC), 2010, pp. 3204-3210, June 2010.

[5] X. Lu, Multi-Agent Reinforcement Learning in Games. PhD thesis, Carleton University, Ottawa, 2012.

[6] K.-H. Hsia and J.-G. Hsieh, "A first approach to fuzzy differential game problem: guarding a territory," Fuzzy Sets and Systems, vol. 55, no. 2, pp. 157 - 167, 1993.

[7] P. Kulkarni, Reinforcement and Systemic Machine Learning for Decision Making. 2012.

[8] R. S. Sutton and A. G. Barto, Introduction to Reinforcement Learning. Cambridge, MA, USA: MIT Press, 1st ed., 1998.

[9] M. Otterlo and M. Wiering, Reinforcement learning: state-of-the-art, vol. 12.; 12. New York; Berlin: Springer, 2012. 
[10] L. Jouffe, "Fuzzy inference system learning by reinforcement methods," Systems, Man, and Cybernetics, Part C: Applications and Reviews, IEEE Transactions on, vol. 28, pp. 338-355, Aug 1998.

[11] G. Philip, "Multi-robot exploration using potential games," Master's thesis, Carleton University, Ottawa, 2013.

[12] E. Uchibe, M. Asada, and K. Hosoda, "Behavior coordination for a mobile robot using modular reinforcement learning," in Intelligent Robots and Systems '96, IROS 96, Proceedings of the 1996 IEEE/RSJ International Conference on, vol. 3, pp. 1329-1336 vol.3, Nov 1996.

[13] S. F. Desouky and H. M. Schwartz, "Self-learning fuzzy logic controllers for pursuitevasion differential games," Robotics and Autonomous Systems, vol. 59, no. 1, pp. $22-33,2011$.

[14] S. Desouky and H. Schwartz, "A novel hybrid learning technique applied to a self-learning multi-robot system," in Systems, Man and Cybernetics, 2009. SMC 2009. IEEE International Conference on, pp. 2616-2623, Oct 2009.

[15] C. Chen, D. Dong, Q. Shi, and Y. Dong, "A quantum reinforcement learning method for repeated game theory," in Computational Intelligence and Security, 2006 International Conference on, vol. 1, pp. 68-72, Nov 2006.

[16] H. Schwartz, Multi-Agent Machine Learning: A Reinforcement Approach. New York, USA: Wiley, 2014.

[17] O. Sigaud and O. Buffet, Markov Decision Processes in Artificial Intelligence. Hoboken: Wiley-ISTE, 2013.

[18] H. S. Chang and SpringerLink, Simulation-based algorithms for Markov decision processes. New York; London: Springer, 2013.

[19] Mausam, A. Kolobov, and Morgan, Planning with Markov decision processes: an AI perspective, vol. 17.; 17. San Rafael, Calif. (1537 Fourth Street, San Rafael, CA 94901 USA): Morgan and Claypool, 2012.

[20] G. Shirazi, P.-Y. Kong, and C.-K. Tham, "Cooperative retransmissions using markov decision process with reinforcement learning," in Personal, Indoor and Mobile Radio Communications, 2009 IEEE 20th International Symposium on, pp. 652-656, Sept 2009. 
[21] A. Lew, H. Mauch, and SpringerLink, Dynamic programming: a computational tool, vol. 38. New York; Berlin: Springer, 2007.

[22] A. Thomas and S. Marcus, "Reinforcement learning for mdps using temporal difference schemes," in Decision and Control, 199\%., Proceedings of the 36th IEEE Conference on, vol. 1, pp. 577-583 vol.1, Dec 1997.

[23] H. Karbasian, M. Ahmadabadi, and B. Araabi, "Improving reinforcement learning using temporal-difference network eurocon2009," in EUROCON 2009, EUROCON '09. IEEE, pp. 1716-1722, May 2009.

[24] C. Watkins and P. Dayan, "Q-learning," Machine Learning, vol. 8, no. 3-4, pp. 279-292, 1992.

[25] J. Liu, S. Liu, H. Wu, and Y. Zhang, "A pursuit-evasion algorithm based on hierarchical reinforcement learning," in Measuring Technology and Mechatronics Automation, 2009. ICMTMA '09. International Conference on, vol. 2, pp. 482486, April 2009.

[26] N. N. Vorobev and S. Kotz, Game theory: lectures for economists and systems scientists, vol. 7. 1977.

[27] X. Song, "Experience generation in tic-tac-toe for general game learning," in Complex, Intelligent and Software Intensive Systems (CISIS), 2012 Sixth International Conference on, pp. 1-5, July 2012.

[28] J.-h. Wang, The Theory of games. Beijing: Tsinghua U. P, 1988.

[29] X. Lu, H. M. Schwartz, and S. Givigi, "Policy Invariance under Reward Transformations for General-Sum Stochastic Games," Journal of Artificial Intelligence Research, 2011.

[30] G. Owen and T.-F. Group, Game theory. San Diego: Academic Press, 1995.

[31] L. C. Thomas, Games, theory, and applications. Chichester; New York: E. Horwood, 1984.

[32] M. L. Littman and C. Szepesvári, "A generalized reinforcement-learning model: Convergence and applications," in ICML, pp. 310-318, 1996.

[33] W. Jintian and Z. Lei, "Application of reinforcement learning in dynamic pricing algorithms," in Automation and Logistics, 2009. ICAL '09. IEEE International Conference on, pp. 419-423, Aug 2009. 
[34] M. Fang, H. Li, and X. Zhang, "A heuristic reinforcement learning based on state backtracking method," in Web Intelligence and Intelligent Agent Technology (WI-IAT), 2012 IEEE/WIC/ACM International Conferences on, vol. 1, pp. 673-678, Dec 2012.

[35] S. Givigi and H. M. Schwartz, "Decentralized strategy selection with learning automata for multiple pursuer-evader games," Adaptive Behavior, vol. 22, no. 4, pp. 221-234, 2014.

[36] L. Galway, D. Charles, and M. Black, "Improving temporal difference game agent control using a dynamic exploration during control learning," in Computational Intelligence and Games, 2009. CIG 2009. IEEE Symposium on, pp. 38-45, Sept 2009.

[37] P. Patel, N. Carver, and S. Rahimi, "Tuning computer gaming agents using qlearning," in Computer Science and Information Systems (FedCSIS), 2011 Federated Conference on, pp. 581-588, Sept 2011.

[38] S. Wender and I. Watson, "Applying reinforcement learning to small scale combat in the real-time strategy game starcraft:broodwar," in Computational Intelligence and Games (CIG), 2012 IEEE Conference on, pp. 402-408, Sept 2012.

[39] Y. Liu, D. Li, and Y. Hu, "Machine learning in adversarial game using flight chess," in Multimedia Information Networking and Security (MINES), 2011 Third International Conference on, pp. 65-68, Nov 2011.

[40] M. van der Ree and M. Wiering, "Reinforcement learning in the game of othello: Learning against a fixed opponent and learning from self-play," in Adaptive Dynamic Programming And Reinforcement Learning (ADPRL), 2013 IEEE Symposium on, pp. 108-115, April 2013.

[41] V. Vassiliades, A. Cleanthous, and C. Christodoulou, "Multiagent reinforcement learning: Spiking and nonspiking agents in the iterated prisoner's dilemma," Neural Networks, IEEE Transactions on, vol. 22, pp. 639-653, April 2011.

[42] X. Lu and H. Schwartz, "Decentralized learning in two-player zero-sum games: A lr-i lagging anchor algorithm," in American Control Conference (ACC), 2011, pp. 107-112, June 2011.

[43] B. Siciliano, L. Sciavicco, L. Villani, and G. Oriolo, Robotics Modelling, Planning and Control. Springer, 2009. 
[44] J.-S. R. Jang, C.-T. Sun, and E. Mizutani, Neuro-Fuzzy and Soft Computing: A Computational Approach to Learning and Machine Intelligence. Prentice Hall, 1996.

[45] Y.-C. Chang, S.-M. Chen, and C.-J. Liau, "Fuzzy interpolative reasoning for sparse fuzzy-rule-based systems based on the areas of fuzzy sets," Fuzzy Systems, IEEE Transactions on, vol. 16, pp. 1285-1301, Oct 2008.

[46] L.-X. Wang, A course in fuzzy systems and control. Upper Saddle River, N.J: Prentice Hall PTR, 1997.

[47] T. Takagi and M. Sugeno, "Fuzzy identification of systems and its applications to modeling and control," Systems, Man and Cybernetics, IEEE Transactions on, vol. SMC-15, pp. 116-132, Jan 1985.

[48] M. J. Er and L. San, "Automatic generation of fuzzy inference systems using incremental-topological-preserving-map-based fuzzy q- learning," in Fuzzy Systems, 2008. FUZZ-IEEE 2008. (IEEE World Congress on Computational Intelligence). IEEE International Conference on, pp. 467-474, June 2008.

[49] H. Berenji, "Fuzzy q-learning: a new approach for fuzzy dynamic programming," in Fuzzy Systems, 1994. IEEE World Congress on Computational Intelligence., Proceedings of the Third IEEE Conference on, pp. 486-491 vol.1, Jun 1994.

[50] S. Wang, A. Panzica, and T. Padir, "Motion control for intelligent ground vehicles based on the selection of paths using fuzzy inference," in Technologies for Practical Robot Applications (TePRA), 2013 IEEE International Conference on, pp. 1-6, April 2013. 